\title{
THERMODYNAMIC FORMALISM FOR RANDOM WEIGHTED COVERING SYSTEMS
}

\author{
JASON ATNIP, GARY FROYLAND, CECILIA GONZÁLEZ-TOKMAN, AND SANDRO VAIENTI
}

\begin{abstract}
We develop for the first time a quenched thermodynamic formalism for random dynamical systems generated by countably branched, piecewise-monotone mappings of the interval that satisfy a random covering condition. Given a random contracting potential $\varphi$ (in the sense of Liverani-Saussol-Vaienti), we prove there exists a unique random conformal measure $\nu_{\varphi}$ and unique random equilibrium state $\mu_{\varphi}$. Further, we prove quasi-compactness of the associated transfer operator cocycle and exponential decay of correlations for $\mu_{\varphi}$. Our random driving is generated by an invertible, ergodic, measure-preserving transformation $\sigma$ on a probability space $(\Omega, \mathscr{F}, m)$; for each $\omega \in \Omega$ we associate a piecewise-monotone, surjective map $T_{\omega}: I \rightarrow I$. We consider general potentials $\varphi_{\omega}: I \rightarrow \mathbb{R} \cup\{-\infty\}$ such that the weight function $g_{\omega}=e^{\varphi_{\omega}}$ is of bounded variation. We provide several examples of our general theory. In particular, our results apply to new examples of linear and non-linear systems including random $\beta$-transformations, randomly translated random $\beta$-transformations, countably branched random Gauss-Renyi maps, random non-uniformly expanding maps (such as intermittent maps and maps with contracting branches) composed with expanding maps, and a large class of random Lasota-Yorke maps.
\end{abstract}

\section{CONTEnts}

1. Introduction 2

2. Setup 4

2.1. Preliminaries on Random Measures 8

2.2. Conditions on the Dynamics 9

2.3. Main Results 12

3. Existence of a Conformal Family of Measures 14

4. Lasota-Yorke Inequalities 18

5. Random Birkhoff Cones and Hilbert Metrics 24

6. Cone Contraction on Good Fibers 27

7. Dealing With Bad Fibers 30

8. Invariant Family of Measures 36

9. Random Measures 47

10. Expected Pressure $\quad 51$

11. Decay of Correlations $\quad 54$

12. Relative Equilibrium States 56

13. Examples 61

13.1. Random $\beta$-Transformations (I) $\quad 62$

13.2. Random $\beta$-Transformations (II) 64

13.3. Randomly Translated Random $\beta$-Transformations 66

13.4. Random Maps With Infinitely Many Branches 68

13.5. Random Non-Uniformly Expanding Maps 69

13.6. Random Lasota-Yorke maps $\quad 71$ 
Acknowledgments 74

$\begin{array}{ll}\text { References } & 75\end{array}$

\section{INTRODUCTION}

Deterministic transitive expansive dynamics $T:[0,1] \rightarrow[0,1]$ or $T: S^{1} \rightarrow S^{1}$ have a well-developed "thermodynamic formalism", whereby the transfer operator with potential $\varphi$ of sufficient regularity has a unique invariant probability measure $\mu$ that is absolutely continuous with respect to a conformal measure, and $\mu$ arises as a maximizer of the sum of the metric entropy and the integral of the potential $[9,46]$ (shifts of finite type, smooth dynamics), [15, 33, 11] (piecewise smooth dynamics), [46, 44] (distance expanding mappings), $[36,11,3,54,12]$ (countable shifts, non-uniformly expanding dynamics).

Thermodynamic formalism for random dynamical systems has been exclusively restricted to the case of continuous random dynamics and maps that can be coded as random shifts. Kifer [29] proved the existence and uniqueness of equilibrium states for smooth expanding dynamics; Khanin and Kifer [28] generalized this to smooth random dynamics that are expanding on average. In parallel work, Bogenschütz [7] proved a variational principle for topological random dynamical systems (the domain $X$ is compact and each $T_{\omega}: X \rightarrow X$ is a homeomorphism), extending a result of Ledrappier and Walters [30] to allow random potentials with measurable dependence $\omega \mapsto \varphi_{\omega}$. For random subshifts of finite type, Bogenschütz and Gundlach [8] and Gundlach [23] proved uniqueness of (random) relative equilibrium states. Thermodynamic formalism for countable random Markov shifts has been investigated in various settings by Denker, Kifer, and Stadlbauer [16], Stadlbauer [49, 50], Roy and Urbański [45], and Mayer and Urbański [38]. Mayer, Skorulski, and Urbański developed distance expanding random mappings in [39], which generalize the works of [29] and [8]. Simmons and Urbański established a variational principle and the existence of a unique relative equilibrium state for these maps in [48]. In the recent articles [52] and [51], the authors considered sequential dynamics given by composition of transfer operators for continuous surjective expanding and non-uniform expanding maps; this allowed them to construct random equilibrium states, and to establish statistical laws.

In the present paper, for the first time, we develop a thermodynamic formalism in the quenched random setting for the difficult situation of discontinuous fiber maps. Furthermore, also for the first time we consider the case of countably branched random maps which cannot be coded by a random shift. We work in the random setting in which one has a complete probability space $(\Omega, \mathscr{F}, m)$ and base dynamical system $\sigma: \Omega \rightarrow \Omega$, which is merely assumed to be invertible, $m$-preserving, and ergodic. For each base configuration $\omega \in \Omega$, we associate a corresponding surjective piecewise monotonic map (possibly with countably many branches) $T_{\omega}:[0,1] \rightarrow[0,1]$. Our random dynamical system on the interval is given by a map cocycle $T_{\omega}^{n}:=T_{\sigma^{n-1}(\omega)} \circ \cdots \circ T_{\sigma(\omega)} \circ T_{\omega}$, which satisfies a random covering condition; we have no Markovian assumptions. Indeed, our maps may have discontinuities and do not necessarily have full branches. The corresponding transfer operator cocycles $\mathcal{L}_{\omega}^{n}:=\mathcal{L}_{\sigma^{n-1}(\omega)} \circ \cdots \circ \mathcal{L}_{\sigma(\omega)} \circ \mathcal{L}_{\omega}$ are weighted using random versions of the contracting potentials introduced in [33].

A first step in producing a thermodynamic formalism in this random setting is the construction of a conformal measure. In the deterministic setting Liverani, Saussol, and Vaienti 
[33] constructed the conformal measure of a piecewise monotonic interval map with a contracting potential $\varphi$ via forward iteration of the transfer operator. This forward iteration is convenient for our random dynamics, and many of the key contributions of our work are the extension of the methodology of [33] to general random piecewise expanding dynamics. In Theorem 2.19 we obtain existence of a fiber-wise collection of conformal measures $\left(\nu_{\omega}\right)_{\omega \in \Omega}$ satisfying $\mathcal{L}_{\omega}^{*} \nu_{\sigma(\omega)}=\lambda_{\omega} \nu_{\omega}$ from following the approach of [33] to extend the functional we obtain via fixed point arguments, similar to those of [25] and [39, Chapter 3], to a random non-atomic Borel probability measure. Existence and uniqueness of a family of invariant functions $\left(q_{\omega}\right)_{\omega \in \Omega}$ satisfying $\mathcal{L}_{\omega} q_{\omega}=\lambda_{\omega} q_{\sigma(\omega)}$, the corresponding invariant measures $\mu_{\omega}=q_{\omega} \nu_{\omega}$, and the uniqueness of the $\nu_{\omega}$, will follow from random cone contraction arguments, which in turn rely on random Lasota-Yorke inequalities. Because our fiber maps $T_{\omega}$ are nonsmooth and may have discontinuities, care is needed when developing the random Lasota-Yorke inequalities; we follow the approach of Buzzi [10], whereby we construct a large measure set of fibers for which we obtain cone contraction in a fixed, uniformly bounded time and then show that the "bad" fibers do not occur often enough to distort the obtained cone contraction too much. We note that if all the maps preserve a common cone, one could attempt to use the recent approach of [26].

From the random cone contraction arguments we immediately obtain, in Theorems 2.20 and 2.21, an exponential convergence of the sequence $\left(\lambda_{\sigma^{-n}(\omega)}^{n}\right)^{-1} \mathcal{L}_{\sigma^{-n}(\omega)}^{n} \mathbb{1}$ to the invariant density $q_{\omega}$ as well as a fiberwise exponential decay of correlations with respect to the invariant measure $\mu_{\omega}$ and the ergodicity of the global random measure $\mu$. Following [48], we define the expected topological pressure of the random contracting potential $\varphi=\left(\varphi_{\omega}\right)_{\omega \in \Omega}$ by $\mathcal{E} P(\varphi)=$ $\int_{\Omega} \log \lambda_{\omega} d m(\omega)$, see also $[4,39,53]$. We then prove, in Theorem 2.23, a variational principle for the expected pressure, a new result for infinitely branched random maps, and then show that the $T$-invariant random measure $\mu$ is in fact the unique relative equilibrium state for the random potential $\varphi$, where $T: \Omega \times I \rightarrow \Omega \times I$ is the induced skew product map.

Our main results are summarized by the following simplified theorem. For a complete statement of our results see Section 2.3.

Theorem. Given a random weighted covering system $\left(\Omega, \sigma, m, I,\left(T_{\omega}\right)_{\omega \in \Omega},\left(\varphi_{\omega}\right)_{\omega \in \Omega}\right)$ (see Definition 2.18), the following hold.

(1) There exists a unique random probability measure $\nu=\left(\nu_{\omega}\right)_{\omega \in \Omega}$ such that for each function $f$ of bounded variation

$$
\nu_{\sigma(\omega)}\left(\mathcal{L}_{\omega} f_{\omega}\right)=\lambda_{\omega} \nu_{\omega}\left(f_{\omega}\right),
$$

where

$$
\lambda_{\omega}:=\nu_{\sigma(\omega)}\left(\mathcal{L}_{\omega} \mathbb{1}_{\omega}\right)
$$

(2) There exists a unique, modulo $\nu$, strictly positive random function $q=\left(q_{\omega}\right)_{\omega \in \Omega}$ of bounded variation such that $\nu_{\omega}\left(q_{\omega}\right)=1$ and

$$
\mathcal{L}_{\omega} q_{\omega}=\lambda_{\omega} q_{\sigma(\omega)}
$$

for $m$-a.e. $\omega \in \Omega$.

(3) The random measure $\mu$, given by $\mu_{\omega}=q_{\omega} \nu_{\omega}$, is a $T$-invariant and ergodic random probability measure. Furthermore, the random measure $\mu=\left(\mu_{\omega}\right)_{\omega \in \Omega}$ exhibits a quenched (forward and backward) exponential decay of correlations and is the unique relative equilibrium state for the random contracting potential $\varphi=\left(\varphi_{\omega}\right)_{\omega \in \Omega}$. 
Our results can treat new classes of random dynamical systems, including random $\beta$ transformations (with no upper bound on the number of branches), randomly translated $\beta$-transformations, infinitely branched random Gauss-Renyi maps, random non-uniformly expanding maps which contain an indifferent fixed point or contracting branches composed with expanding maps, and a very broad class of random Lasota-Yorke maps.

An outline of the paper is as follows. Formal definitions, assumptions, and other properties of our random systems are described in Section 2, where we also state our main results. In Section 3 we prove the existence of a non-atomic random conformal measure. Section 4 develops random Lasota-Yorke inequalities for our random contracting potentials in terms of variation and the random conformal measure and in Section 5 we construct the corresponding random cones. Section 6 proves that for a large "Good" subset of $\Omega_{G} \subset \Omega$, a constant number of iterates of the transfer operator beginning at $\omega \in \Omega_{G}$ yields a cone contraction. The remaining "Bad" $\omega$-fibers are dealt with in Section 7. Using Hilbert metric contraction arguments, Section 8 brings together the estimates from Sections 4, 5, 6, and 7 to prove our main technical lemma (Lemma 8.1), which is then used to prove (i) the existence of a random invariant density and (ii) that convergence to this invariant density occurs at an exponential rate along a subsequence. Section 9 verifies that the random families of conformal measures, invariant densities, and invariant measures are indeed measurable with respect to $\omega$. We also prove the uniqueness of the invariant density found in Section 8. In Section 10 we utilize the measurability produced in Section 9 to introduce the expected pressure and then give more refined versions of the exponential convergence results of Section 8 as well as prove the uniqueness of the random conformal and invariant measures. In Section 11 we use the results of Section 10 to prove an exponential decay of correlations for the random invariant measures from Section 9 in both the forward and backward directions. In Section 12 we prove a variational principle and show that the unique invariant measure established in Section 9 is in fact a relative equilibrium state. Finally, in Section 13, we apply our results to new classes of random dynamical systems with detailed calculations.

\section{SETUP}

We begin with a complete probability space $(\Omega, \mathscr{F}, m)$ and suppose that $\sigma: \Omega \rightarrow \Omega$ is an ergodic, invertible map, which preserves the measure $m$, i.e.

$$
m \circ \sigma^{-1}=m \text {. }
$$

Let $I$ denote a compact interval in $\mathbb{R},{ }^{1}$ and for each $\omega \in \Omega$ we consider a map

$$
T_{\omega}: I \longrightarrow I
$$

such that there exists a countable partition $Z_{\omega}^{*}$ of $I$ such that each $Z \in Z_{\omega}^{*}$ satisfies the following:

(i) $T_{\omega}: I \rightarrow I$ is onto,

(ii) $T_{\omega}(Z)$ is an interval,

(iii) $\left.T_{\omega}\right|_{Z}$ is continuous and strictly monotone.

\footnotetext{
${ }^{1}$ Our theory works equally well if $I$ is taken to be an uncountable, totally ordered, order-complete set as in [33]. In this setting the order topology makes $I$ compact. We choose to work under the assumption that $I$ is a compact interval in $\mathbb{R}$ for ease of exposition.
} 
Let $D_{\omega}$ be the set of discontinuities of $T_{\omega}$, i.e. the collection of all endpoints of intervals $Z \in Z_{\omega}^{*}$. Denote by $\mathcal{S}_{\omega}$ the singular set of $T_{\omega}$ which is defined by

$$
\mathcal{S}_{\omega}:=\bigcup_{k \geq 0} T_{\omega}^{-k}\left(D_{\sigma^{k}(\omega)}\right)
$$

Then $\mathcal{S}_{\omega}$ is clearly backward $T_{\omega}$ invariant in the sense that

$$
T_{\omega}^{-1}\left(\mathcal{S}_{\sigma(\omega)}\right) \subseteq \mathcal{S}_{\omega}
$$

Notice that for each $\omega \in \Omega, \mathcal{S}_{\omega}$ is countable. For each $\omega \in \Omega$ let

$$
X_{\omega}:=I \backslash \mathfrak{S}_{\omega},
$$

which naturally inherits the subspace topology of $I$. In the sequel, by an interval $J \subseteq X_{\omega}$, we of course mean $J=\tilde{J} \cap X_{\omega}$ for some interval $\tilde{J} \subseteq I$. By virtue of (2.2), we have that

$$
T_{\omega}\left(X_{\omega}\right) \subseteq X_{\sigma(\omega)} .
$$

In what follows we will consider the dynamics of the maps

$$
\left.T_{\omega}\right|_{X_{\omega}}: X_{\omega} \longrightarrow X_{\sigma(\omega)} \text {. }
$$

We note that the maps $T_{\omega}$ are continuous with respect to the topology on $X_{\omega}$. We consider the global dynamics of the map $T: \Omega \times I \rightarrow \Omega \times I$ given by

$$
T(\omega, x)=\left(\sigma(\omega), T_{\omega}(x)\right) .
$$

Letting

$$
\mathcal{J}:=\bigcup_{\omega \in \Omega}\{\omega\} \times X_{\omega}
$$

we may also consider the global dynamics $\left.T\right|_{\mathfrak{J}}: \mathcal{J} \rightarrow \mathcal{J}$. For each $\omega \in \Omega$ we let

$$
Z_{\omega}^{(1)}:=\left\{Z \cap X_{\omega}: Z \in Z_{\omega}^{*}\right\}
$$

Now, for $n>1$ let

$$
z_{\omega}^{(n)}:=\bigvee_{j=0}^{n-1} T_{\omega}^{-j}\left(z_{\sigma^{j}(\omega)}^{(1)}\right)
$$

Then $Z_{\omega}^{(n)}$ consists of countably many non-degenerate subintervals which are open with respect to $X_{\omega}$. Now, given $Z \in Z_{\omega}^{(n)}$, we denote by

$$
T_{\omega, Z}^{-n}: T_{\omega}^{n}(Z) \longrightarrow Z
$$

the inverse branch of $T_{\omega}^{n}$ which takes $T_{\omega}^{n}(x)$ to $x$ for each $x \in Z$. We will assume that the partitions $Z_{\omega}^{*}$ are generating, i.e.

$$
\bigvee_{n=1}^{\infty} z_{\omega}^{(n)}=\mathscr{B}
$$

where $\mathscr{B}=\mathscr{B}(I)$ denotes the Borel $\sigma$-algebra of $I$.

Let $\mathcal{B}(I)$ denote the set of all bounded real-valued functions on $I$ and for each $f \in \mathcal{B}(I)$ and each $A \subseteq I$ let

$$
\operatorname{var}_{A}(f):=\sup \left\{\sum_{j=0}^{k-1}\left|f\left(x_{j+1}\right)-f\left(x_{j}\right)\right|: x_{0}<x_{1}<\ldots x_{k}, x_{j} \in A \text { for all } 0 \leq j \leq k\right\}
$$


where the supremum is taken over all finite sets $\left\{x_{j}\right\} \subseteq A$, denote the variation of $f$ over $A$. We let

$$
\mathrm{BV}(I):=\left\{f \in \mathcal{B}(I): \operatorname{var}_{I}(f)<\infty\right\}
$$

denote the set of functions of bounded variation on $I$. Let $\|f\|_{\infty}$ and

$$
\|f\|_{\mathrm{BV}}:=\operatorname{var}(f)+\|f\|_{\infty}
$$

be norms on the respective Banach spaces $\mathcal{B}(I)$ and $\mathrm{BV}(I)$.

Let $\mathcal{B}\left(X_{\omega}\right)$ be the set of all bounded real-valued functions on $X_{\omega}$ and let $\mathrm{BV}\left(X_{\omega}\right)$ be the set of all functions of bounded variation on $X_{\omega}$, i.e. all functions $f \in \mathcal{B}\left(X_{\omega}\right)$ such that $\operatorname{var}_{X_{\omega}}(f)<\infty$. For a function $f: \Omega \times I \rightarrow \mathbb{R}$ we let $f_{\omega}:=\left.f\right|_{\{\omega\} \times I}: I \rightarrow \mathbb{R}$ for each $\omega \in \Omega$. In particular, we have that

$$
\mathbb{1}_{\omega}:=\mathbb{1}_{X_{\omega}} .
$$

Note that if $f \in \mathcal{B}(I)$, then $f_{\omega} \in \mathcal{B}\left(X_{\omega}\right)$ and if $f \in \mathcal{B}(I)$ then $f_{\omega} \in \operatorname{BV}\left(X_{\omega}\right)$ with $\operatorname{var}_{X_{\omega}}(f) \leq$ $\operatorname{var}_{I}(f)$. For this reason we will generally write $\operatorname{var}\left(f_{\omega}\right)$, suppressing the set dependence, when dealing with the variation, either on the entire interval $I$ or fiber space $X_{\omega}$, unless more care is needed.

Definition 2.1. We say that a function $f: \Omega \times I \rightarrow \mathbb{R}$ is random bounded if

(i) $f_{\omega} \in \mathcal{B}(I)$ for each $\omega \in \Omega$,

(ii) for each $x \in I$ the function $\Omega \ni \omega \mapsto f_{\omega}(x)$ is measurable,

(iii) the function $\Omega \ni \omega \mapsto\left\|f_{\omega}\right\|_{\infty}$ is measurable.

Let $\mathcal{B}_{\Omega}(I)$ denote the collection of all random bounded functions on $\Omega \times I$, and let

$$
\mathcal{B}_{\Omega}^{1}(I)=\left\{f \in \mathcal{B}_{\Omega}:\left\|f_{\omega}\right\|_{\infty} \in L_{m}^{1}(\Omega)\right\} .
$$

The set $\mathcal{B}_{\Omega}^{1}(I)$ becomes a Banach space when taken together with norm

$$
|f|_{\infty}:=\int_{\Omega}\left\|f_{\omega}\right\|_{\infty} d m(\omega) .
$$

We say that a function $f \in \mathcal{B}_{\Omega}(I)$ is of random bounded variation if $f_{\omega} \in \mathrm{BV}(I)$ for each $\omega \in \Omega$. We let $\mathrm{BV}_{\Omega}(I)$ denote the set of all random bounded variation functions and let

$$
\mathrm{BV}_{\Omega}^{l}(I):=\left\{f \in \mathrm{BV}_{\Omega}(I):\left\|f_{\omega}\right\|_{\infty}, \log \operatorname{var}\left(f_{\omega}\right), \log \inf \left|f_{\omega}\right| \in L_{m}^{1}(\Omega)\right\} .
$$

For functions $f: \Omega \times I \rightarrow \mathbb{R}$ and $F: \Omega \rightarrow \mathbb{R}$ we let

$$
S_{n, T}\left(f_{\omega}\right):=\sum_{j=0}^{n-1} f_{\sigma^{j}(\omega)} \circ T_{\omega}^{j} \quad \text { and } \quad S_{n, \sigma}(F):=\sum_{j=0}^{n-1} F \circ \sigma^{j}
$$

denote the Birkhoff sums of $f$ and $F$ with respect to $T$ and $\sigma$ respectively. We will consider a potential of the form $\varphi: \Omega \times I \rightarrow \mathbb{R} \cup\{-\infty\}$ and for each $n \geq 1$ we consider the weight $g^{(n)}: \Omega \times I \rightarrow \mathbb{R}$ whose disintegrations are given by

$$
g_{\omega}^{(n)}:=\exp \left(S_{n, T}\left(\varphi_{\omega}\right)\right)
$$

for each $\omega \in \Omega$. We define the (Perron-Frobenius) transfer operator, $\mathcal{L}_{\varphi, \omega}: \mathcal{B}\left(X_{\omega}\right) \rightarrow$ $\mathcal{B}\left(X_{\sigma(\omega)}\right)$, with respect to the potential $\varphi: \Omega \times I \rightarrow \mathbb{R} \cup\{-\infty\}$, by

$$
\mathcal{L}_{\varphi, \omega}(f)(x)=\mathcal{L}_{\omega}(f)(x):=\sum_{y \in T_{\omega}^{-1}(x)} g_{\omega}^{(1)}(y) f(y) ; \quad f \in \mathcal{B}\left(X_{\omega}\right), x \in X_{\sigma(\omega)} .
$$


We define the global operator $\mathcal{L}: \mathcal{B}_{\Omega}(I) \rightarrow \mathcal{B}_{\Omega}(I)$ by

$$
(\mathcal{L} f)_{\omega}:=\mathcal{L}_{\sigma^{-1}(\omega)} f_{\sigma^{-1}(\omega)}
$$

Inducting on $n$ gives that the iterates $\mathcal{L}_{\omega}^{n}: \mathcal{B}\left(X_{\omega}\right) \rightarrow \mathcal{B}\left(X_{\sigma^{n}(\omega)}\right)$ are given by

$$
\begin{aligned}
\mathcal{L}_{\varphi, \omega}^{n}(f)(x) & =\left(\mathcal{L}_{\sigma^{n-1}(\omega)} \circ \cdots \circ \mathcal{L}_{\omega}\right)(f)(x) \\
& =\sum_{y \in T_{\omega}^{-n}(x)} g_{\omega}^{(n)}(y) f(y) ; \quad f \in \mathcal{B}\left(X_{\omega}\right), x \in X_{\sigma^{n}(\omega)} .
\end{aligned}
$$

For each $\omega \in \Omega$ we let $\mathcal{B}^{*}\left(X_{\omega}\right)$ and $\mathrm{BV}^{*}\left(X_{\omega}\right)$ denote the respective dual spaces of $\mathcal{B}\left(X_{\omega}\right)$ and $\operatorname{BV}\left(X_{\omega}\right)$. We let $\mathcal{L}_{\omega}^{*}: \mathcal{B}^{*}\left(X_{\sigma(\omega)}\right) \rightarrow \mathcal{B}^{*}\left(X_{\omega}\right)$ be the dual transfer operator.

To ensure that these operators $\mathcal{L}_{\omega}$ are well defined and that the sum in (2.5) does in fact converge, we will need some additional assumptions on the potential $\varphi$.

Definition 2.2. We will say that a potential $\varphi: \Omega \times I \rightarrow \mathbb{R} \cup\{-\infty\}$ is summable if for $m$-a.e. $\omega \in \Omega$ we have

(SP1) inf $\left.g_{\omega}^{(1)}\right|_{Z}>0$ for all $Z \in \mathcal{Z}_{\omega}^{(1)}$

(SP2) $g_{\omega}^{(1)} \in \mathrm{BV}(I)$,

(SP3) $S_{\omega}^{(1)}:=\sum_{Z \in Z_{\omega}^{*}} \sup _{Z} g_{\omega}^{(1)}<\infty$.

Remark 2.3. Note that if $\# Z_{\omega}^{(1)}<\infty$ and $\varphi_{\omega} \in \mathrm{BV}(I)$ for each $\omega \in \Omega$ then (SP1)-(SP3) are immediate. Furthermore, as $\varphi_{\omega} \in \mathcal{B}(I)$ we would also have that inf $g_{\omega}^{(n)}>0$ for each $\omega \in \Omega$ and $n \in \mathbb{N}$.

Note that (SP3) implies that the transfer operator $\mathcal{L}_{\omega}: \mathcal{B}\left(X_{\omega}\right) \rightarrow \mathcal{B}\left(X_{\sigma(\omega)}\right)$ is well defined. The following lemma shows that the operator $\mathcal{L}_{\omega}^{n}: \mathcal{B}\left(X_{\omega}\right) \rightarrow \mathcal{B}\left(X_{\sigma^{n}(\omega)}\right)$ is well defined for all $n \geq 1$.

Lemma 2.4. Given a summable potential $\varphi: \Omega \times I \rightarrow \mathbb{R} \cup\{-\infty\}$, for all $n \in \mathbb{N}$ and m-a.e. $\omega \in \Omega$ we have

(i) $g_{\omega}^{(n)} \in \mathrm{BV}(I)$,

(ii) $S_{\omega}^{(n)}:=\sum_{Z \in Z_{\omega}^{(n)}} \sup _{Z} g_{\omega}^{(n)}<\infty$.

Proof. We will prove each of the claims via induction. To prove (i), we first note that by (SP2) $g_{\omega}^{(1)} \in \mathrm{BV}(I)$. Now suppose that $g_{\omega}^{(j)} \in \mathrm{BV}(I)$ for each $\omega \in \Omega$ and all $1 \leq j \leq n$. Now, note that we may write

$$
g_{\omega}^{(n+1)}=g_{\omega}^{(1)} \cdot g_{\sigma(\omega)}^{(n)} \circ T_{\omega} .
$$

Then,

$$
\begin{aligned}
\operatorname{var}\left(g_{\omega}^{(n+1)}\right) & \leq \sum_{Z \in \mathcal{Z}_{\omega}^{(1)}}\left(\operatorname{var}_{Z}\left(g_{\omega}^{(n+1)}\right)+2 \sup _{Z} g_{\omega}^{(n+1)}\right) \\
& \leq \sum_{Z \in \mathcal{Z}_{\omega}^{(1)}}\left(\operatorname{var}_{Z}\left(g_{\omega}^{(1)}\right) \sup _{T_{\omega}(Z)} g_{\sigma(\omega)}^{(n)}+\sup _{Z} g_{\omega}^{(1)} \operatorname{var}_{T_{\omega}(Z)}\left(g_{\sigma(\omega)}^{(n)}\right)+2 \sup _{Z} g_{\omega}^{(1)} \sup _{T_{\omega}(Z)} g_{\sigma(\omega)}^{(n)}\right)
\end{aligned}
$$




$$
\begin{aligned}
& \leq\left\|g_{\sigma(\omega)}^{(n)}\right\|_{\mathrm{BV}} \sum_{Z \in \mathcal{Z}_{\omega}^{(1)}}\left(\operatorname{var}_{Z} g_{\omega}^{(1)}+\sup _{Z} g_{\omega}^{(1)}+2 \sup _{Z} g_{\omega}^{(1)}\right) \\
& \leq\left(3 S_{\omega}^{(1)}+\operatorname{var}\left(g_{\omega}^{(1)}\right)\right)\left\|g_{\sigma(\omega)}^{(n)}\right\|_{\mathrm{BV}} .
\end{aligned}
$$

To see (ii), we begin by noting that (SP3) implies that $S_{\omega}^{(1)}<\infty$. Now, suppose that $S_{\omega}^{(j)}<\infty$ for each $\omega \in \Omega$ and all $1 \leq j \leq n$. Now, note that we may also rewrite $g_{\omega}^{(n+1)}$ as

$$
g_{\omega}^{(n+1)}=g_{\omega}^{(n)} \cdot g_{\sigma^{n}(\omega)}^{(1)} \circ T_{\omega}^{n} .
$$

Then,

$$
\begin{aligned}
S_{\omega}^{(n+1)} & =\sum_{Z \in \mathcal{Z}_{\omega}^{(n+1)}} \sup _{Z} g_{\omega}^{(n+1)} \leq \sum_{Z \in \mathcal{Z}_{\omega}^{(n+1)}} \sup _{Z} g_{\omega}^{(n)} \sup _{T_{\omega}^{n}(Z)} g_{\sigma^{n}(\omega)}^{(1)} \\
& \leq \sum_{Z^{\prime} \in \mathcal{Z}_{\omega}^{(n)}} \sum_{\substack{Z \in \mathcal{Z}_{\omega}^{(n+1)} \\
Z \subseteq Z^{\prime}}} \sup _{Z^{\prime}} g_{\omega}^{(n)} \sup _{T_{\omega}^{n}(Z)} g_{\sigma^{n}(\omega)}^{(1)} \\
& \leq \sum_{Z^{\prime} \in \mathcal{Z}_{\omega}^{(n)}} \sup _{Z^{\prime}} g_{\omega}^{(n)} \cdot \sum_{Z^{\prime \prime} \in \mathcal{Z}_{\sigma^{n}(\omega)}^{(1)}} \sup _{Z^{\prime \prime}} g_{\sigma^{n}(\omega)}^{(1)} \\
& \leq S_{\omega}^{(n)} \cdot S_{\sigma^{n}(\omega)}^{(1)}<\infty
\end{aligned}
$$

and thus we are done.

2.1. Preliminaries on Random Measures. Given a measurable space $Y$, we let $\mathcal{P}(Y)$ denote the collection of all Borel probability measures on $Y$. Recall that $\mathscr{B}$ denotes the Borel $\sigma$-algebra of $I$, and for each $\omega \in \Omega$, let $\mathscr{B}_{\omega}$ denote the Borel $\sigma$-algebra of $X_{\omega}$, i.e. the $\sigma$-algebra $\mathscr{B}$ restricted to the set $X_{\omega}$. Now, let $\mathscr{F} \otimes \mathscr{B}$ denote the product $\sigma$-algebra of $\mathscr{B}$ and $\mathscr{F}$ on $\Omega \times I$.

Let $\mathcal{P}_{m}(\Omega \times I)$ denote the set of all probability measures $\mu$ on $\Omega \times I$ that have marginal $m$, i.e.

$$
\mathcal{P}_{m}(\Omega \times I):=\left\{\mu \in \mathcal{P}(\Omega \times I): \mu \circ \pi_{\Omega}^{-1}=m\right\},
$$

where $\pi_{\Omega}: \Omega \times X \rightarrow \Omega$ is the projection onto the first coordinate.

Definition 2.5. A map $\mu: \Omega \times \mathscr{B} \rightarrow[0,1]$ with $\Omega \times \mathscr{B} \ni(\omega, B) \mapsto \mu_{\omega}(B)$ is said to be a random probability measure on $I$ if

(1) for every $B \in \mathscr{B}$, the map $\Omega \ni \omega \mapsto \mu_{\omega}(B) \in[0,1]$ is measurable,

(2) for $m$-a.e. $\omega \in \Omega$, the map $\mathscr{B} \ni B \mapsto \mu_{\omega}(B) \in[0,1]$ is a Borel probability measure.

We let $\mathcal{P}_{\Omega}(I)$ denote the set of all random probability measures on $I$. We will frequently denote a random measure $\mu$ by $\left(\mu_{\omega}\right)_{\omega \in \Omega}$.

The following proposition, which summarizes results of Crauel [14], shows that the collection $\mathcal{P}_{m}(\Omega \times I)$ can be canonically identified with the collection $\mathcal{P}_{\Omega}(I)$ of all random probability measures on $I$.

Proposition 2.6 ([14], Propositions 3.3, 3.6). For each $\mu \in \mathcal{P}_{m}(\Omega \times I)$ there exists a unique random probability measure $\left(\mu_{\omega}\right)_{\omega \in \Omega} \in \mathcal{P}_{\Omega}(I)$ such that

$$
\int_{\Omega \times I} f(\omega, x) d \mu(\omega, x)=\int_{\Omega} \int_{I} f(\omega, x) d \mu_{\omega}(x) d m(\omega)
$$


for every bounded measurable function $f: \Omega \times I \rightarrow \mathbb{R}$.

Conversely, if $\left(\mu_{\omega}\right)_{\omega \in \Omega} \in \mathcal{P}_{\Omega}(I)$ is a random probability measure on $I$, then for every bounded measurable function $f: \Omega \times I \rightarrow \mathbb{R}$ the function

$$
\Omega \ni \omega \longmapsto \int_{I} f(\omega, x) d \mu_{\omega}(x)
$$

is measurable and

$$
\mathscr{F} \otimes \mathscr{B} \ni A \longmapsto \int_{\Omega} \int_{I} \mathbb{1}_{A}(\omega, x) d \mu_{\omega}(x) d m(\omega)
$$

defines a probability measure in $\mathcal{P}_{m}(\Omega \times I)$.

2.2. Conditions on the Dynamics. We now give a definition of random covering similar to the covering properties of [10] and [33].

Definition 2.7. We will say that our system has random covering if for each $\omega \in \Omega$ and each $J \subseteq I$ there exists $M_{\omega}=M_{\omega}(J) \in \mathbb{N}$ such that for all $n \geq M_{\omega}(J)$ there exists a constant $C_{\omega, n}(J)>0$ such that

$$
\inf _{X_{\sigma}(\omega)} \mathcal{L}_{\omega}^{n_{1}} \mathbb{1} J \geq C_{\omega, n}(J) .
$$

Remark 2.8. Note that random covering implies that for all intervals $J \subseteq I$ there exists $M_{\omega}=M_{\omega}(J) \in \mathbb{N}$ such that

$$
T_{\omega}^{M_{\omega}}(J) \supseteq X_{\sigma^{M_{\omega}}(\omega)},
$$

and also that for all intervals $J \subseteq X_{\omega}$ there exists $M_{\omega} \in \mathbb{N}$ such that

$$
T_{\omega}^{M_{\omega}}(J)=X_{\sigma^{M \omega}(\omega)} .
$$

Furthermore, note that if the partition $z_{\omega}^{*}$ is finite then (2.6) and (2.7) are equivalent to $(\mathrm{RC})$.

We now concern ourselves with special finite partitions of $I$. For each $n \in \mathbb{N}$, each $\omega \in \Omega$, $\hat{\alpha} \geq 0$, and $\hat{\gamma} \geq 1$ we let $\mathcal{P}_{\omega, n}(\hat{\alpha}, \hat{\gamma})$ be a finite partition of $I$ such that for each $P \in \mathcal{P}_{\omega, n}(\hat{\alpha}, \hat{\gamma})$ the following hold:

$$
\begin{aligned}
& \sum_{\substack{Z \in Z_{\omega}^{(n)} \\
Z \cap P \neq \emptyset}} \sup _{\substack{Z \\
Z \cap P}} g_{\omega}^{(n)} \leq \hat{\gamma}\left\|g_{\omega}^{(n)}\right\|_{\infty} \\
&
\end{aligned}
$$

Remark 2.9. Note that the for certain potentials we may be able to satisfy (P1) and (P2) for $\hat{\alpha}=0$ and $\hat{\gamma}=1$. Indeed, if $z_{\omega}^{(n)}$ is finite and if the partition $z_{\omega}^{(n)}$ satisfies (P1) for $\hat{\alpha} \geq 0$, then we may take $\hat{\gamma}=1$ and $\mathcal{P}_{\omega, n}(\hat{\alpha}, 1)=Z_{\omega}^{(n)}$, as (P2) holds trivially for $\mathcal{Z}_{\omega}^{(n)}$. Furthermore, if the weight functions $g_{\omega}$ are constant then we may take $\hat{\alpha}=0$.

The following lemma gives conditions for such partitions to exist.

Lemma 2.10. If $\hat{\alpha}>1$ and $\hat{\gamma} \geq 1$ then for each $n \in \mathbb{N}$ and $\omega \in \Omega$ there exists a partition $\mathcal{P}_{\omega, n}(\hat{\alpha}, \hat{\gamma})$ which satisfies conditions (P1) and (P2). 
Proof. Following Lemma 6 of [47], for any $\varepsilon>0$ we can find a finite partition $\mathcal{P}$ such that

$$
\operatorname{var}_{P}\left(g_{\omega}^{(n)}\right) \leq\left\|g_{\omega}^{(n)}\right\|_{\infty}+\varepsilon
$$

for each $P \in \mathcal{P}$. Thus for any $\hat{\alpha}>1$ we can find a finite partition $\mathcal{P}_{\omega, n}(\hat{\alpha})$ such that

$$
\operatorname{var}_{P}\left(g_{\omega}^{(n)}\right) \leq \hat{\alpha}\left\|g_{\omega}^{(n)}\right\|_{\infty}
$$

for each $P \in \mathcal{P}_{\omega, n}(\hat{\alpha})$. Now, following the argument in the proof of Sub-Lemma 4.1.1 of [33], for $\beta \geq 1$ we can then refine the partition $\mathcal{P}_{\omega, n}(\hat{\alpha})$ with $z_{\omega}^{(n)}$ to obtain a finite partition $\mathcal{P}_{\omega, n}(\hat{\alpha}, \hat{\gamma})$ such that

$$
\sum_{\substack{Z \in Z_{\omega}^{(n)} \\ Z \cap P \neq \emptyset}} \sup _{Z \cap P} g_{\omega}^{(n)} \leq \hat{\gamma}\left\|g_{\omega}^{(n)}\right\|_{\infty}
$$

for each $P \in \mathcal{P}_{\omega, n}(\hat{\alpha}, \hat{\gamma})$ since $S_{\omega}^{(n)}<\infty$.

Remark 2.11. For our general theory we are only concerned that there exist $\hat{\alpha} \geq 0$ and $\hat{\gamma} \geq 1$ and partitions $\mathcal{P}_{\omega, n}(\hat{\alpha}, \hat{\gamma})^{2}$ such that (P1) and (P2) are satisfied. However, as we will see in Section 13, the choice of $\hat{\alpha}$ and $\hat{\gamma}$ directly impacts the amount of work necessary to verify our hypotheses for examples. So, finding (nearly) optimal values for $\hat{\alpha}$ and $\hat{\gamma}$ may be necessary in practice.

Now, fixing $\hat{\alpha} \geq 0$ and $\hat{\gamma} \geq 1$ such that (P1) and (P2) hold for the partitions $\mathcal{P}_{\omega, n}(\hat{\alpha}, \hat{\gamma})$, since $Z_{\omega}^{*}$ is generating, i.e. assumption (GP), for each $P \in \mathcal{P}_{\omega, n}(\hat{\alpha}, \hat{\gamma})$ there exists a least number $N_{\omega, n}(P) \in \mathbb{N}$ and a measurable choice

$$
J(P) \in Z_{\omega}^{\left(N_{\omega, n}(P)\right)}
$$

with $J(P) \subseteq P$.

We will assume the following measurability and integrability conditions on our system.

(M1) The map $T: \Omega \times I \rightarrow \Omega \times I$ is measurable.

(M2) For $f \in \mathrm{BV}_{\Omega}(I)$ we have $\mathcal{L} f \in \mathrm{BV}_{\Omega}(I)$.

(M3) $\log S_{\omega}^{(1)} \in L_{m}^{1}(\Omega)$,

(M4) $\log \inf \mathcal{L}_{\omega} \mathbb{1}_{\omega} \in L_{m}^{1}(\Omega)$,

(M5) $\min _{P \in \mathcal{P}_{\omega, n}(\hat{\alpha}, \hat{\gamma})} \log \inf _{J(P)} g_{\omega}^{M_{\omega}(J(P))} \in L_{m}^{1}(\Omega)$ for each $n \in \mathbb{N}$,

(M6) $\max _{P \in \mathcal{P}_{\omega, n}(\hat{\alpha}, \hat{\gamma})} \log \left\|\mathcal{L}_{\omega}^{M_{\omega}(J(P))} \mathbb{1}_{\omega}\right\|_{\infty} \in L_{m}^{1}(\Omega)$ for each $n \in \mathbb{N}$.

Remark 2.12. Note that condition (M4) implies that $\inf \mathcal{L}_{\omega} \mathbb{1}_{\omega}>0$ for $m$-a.e. $\omega \in \Omega$. Furthermore,although condition (M4) may seem restrictive, the following examples show that it can be easily checked under mild assumptions.

(a) If $z_{\omega}^{*}$ is finite for each $\omega \in \Omega$ with $\varphi_{\omega} \in \mathrm{BV}(I)$ and $\log \inf g_{\omega} \in L_{m}^{1}(\Omega)$, then (M4) holds.

\footnotetext{
${ }^{2}$ In [33] they consider the case $\hat{\alpha}=4$ and $\hat{\gamma}=2$.
} 
(b) (M4) holds if for each $\omega \in \Omega$ there exists a finite collection $Z_{\omega, 1}, \ldots, Z_{\omega, n_{\omega}} \in Z_{\omega}^{*}$ with $T_{\omega}\left(\cup_{j=1}^{n_{\omega}} Z_{\omega, j}\right)=I$ such that $\left.\log \inf g_{\omega}\right|_{\cup_{j=1}^{n_{\omega}} Z_{\omega, j}} \in L_{m}^{1}(\Omega)$. In particular, (M4) holds if for each $\omega$ there exists some $Z_{\omega} \in Z_{\omega}^{(1)}$ which supports a full branch such that $\left.\log \inf g_{\omega}\right|_{Z_{\omega}} \in L_{m}^{1}(\Omega)$.

Given a a real-valued function $f$, we let $f^{+}, f^{-}$denote the positive and negative parts of $f$ respectively with $f^{+}, f^{-} \geq 0$ and $f=f^{+}-f^{-}$. The following lemma now follows from the previous assumptions.

Lemma 2.13. For each $n \in \mathbb{N}$ we have the following:

(i) $\log \inf \mathcal{L}_{\omega}^{n_{\omega}} \mathbb{1}_{\omega} \in L_{m}^{1}(\Omega)$,

(ii) $\log S_{\omega}^{(n)} \in L_{m}^{1}(\Omega)$,

(iii) $\log \left\|\mathcal{L}_{\omega}^{n} \mathbb{1}_{\omega}\right\|_{\infty} \in L_{m}^{1}(\Omega)$,

(iv) $\log ^{+}\left\|g_{\omega}^{(n)}\right\|_{\infty} \in L_{m}^{1}(\Omega)$

(v) $\left(\sup \varphi_{\omega}\right)^{+} \in L_{m}^{1}(\Omega)$.

Proof. We begin by noting that (M2) implies that the functions $\omega \mapsto \inf \mathcal{L}_{\omega} \mathbb{1}_{\omega}, \omega \mapsto \sup \varphi_{\omega}$ (and consequently $\omega \mapsto\left\|g_{\omega}^{(1)}\right\|_{\infty}$ ), and $\omega \mapsto\left\|\mathcal{L}_{\omega} \mathbb{1}_{\omega}\right\|_{\infty}$ are all $m$-measurable. Now we note that the submultiplicativity of $\left\|g_{\omega}^{(n)}\right\|_{\infty}$ gives

$$
0 \leq \log ^{+}\left\|g_{\omega}^{(n)}\right\|_{\infty} \leq \sum_{j=0}^{n-1} \log ^{+}\left\|g_{\sigma^{j}(\omega)}^{(1)}\right\|_{\infty}=\sum_{j=0}^{n-1}\left(\sup \varphi_{\sigma^{j}(\omega)}\right)^{+} \leq \sum_{j=0}^{n-1} \log S_{\sigma^{j}(\omega)}^{(1)}
$$

using (M3) we obtain (iv) and (v). Similarly, the submultiplicativity of $S_{\omega}^{(n)}$ implied by the proof of Lemma 2.4 item (ii) and supermultiplicativity of inf $\mathcal{L}_{\omega}^{n} \mathbb{1}_{\omega}$ yields

$$
\sum_{j=0}^{n-1} \log \inf \mathcal{L}_{\sigma^{j}(\omega)} \mathbb{1}_{\sigma^{j}(\omega)} \leq \log \inf \mathcal{L}_{\omega}^{n} \mathbb{1}_{\omega} \leq \log \left\|\mathcal{L}_{\omega}^{n} \mathbb{1}_{\omega}\right\|_{\infty} \leq \log S_{\omega}^{(n)} \leq \sum_{j=0}^{n-1} \log S_{\sigma^{j}(\omega)}^{(1)},
$$

and thus we obtain (i)-(iii) by (M3) and (M4). As both the right and left-hand sides of (2.11) and (2.12) are $m$-integrable, we are done.

Remark 2.14. Note that since the covering times $M_{\omega}(J(P))$ may change with $\omega$, our assumption (M6) does not necessarily follow from Lemma 2.13.

The next definition, which is an extension of [33, Definition 3.4] into the random setting, will be essential in the sequel.

Definition 2.15. A summable potential $\varphi: \Omega \times I \rightarrow \mathbb{R} \cup\{-\infty\}$ is said to be contracting if for $m$-a.e. $\omega \in \Omega$ we have

$$
\lim _{n \rightarrow \infty} \frac{1}{n} \log \left\|g_{\omega}^{(n)}\right\|_{\infty}<\lim _{n \rightarrow \infty} \frac{1}{n} \log \inf \mathcal{L}_{\omega}^{n} \mathbb{1}_{\omega}{ }^{3}
$$

\footnotetext{
${ }^{3}$ Note that these limits exist by the subadditive ergodic theorem due to the submultiplicativity and supermultiplicativity of the respective sequences $\left\{\omega \mapsto\left\|g_{\omega}^{(n)}\right\|_{\infty}\right\}_{n \in \mathbb{N}}$ and $\left\{\omega \mapsto \inf \mathcal{L}_{\omega}^{n} \mathbb{1}_{\omega}\right\}_{n \in \mathbb{N}}$. Furthermore, the limits are constant $m$-a.e. and the left-hand limit may be equal to $-\infty$.
} 
and there exists $N \in \mathbb{N}$ such that

$$
-\infty<\int_{\Omega} \log \frac{\left\|g_{\omega}^{(N)}\right\|_{\infty}}{\inf \mathcal{L}_{\omega}^{N} \mathbb{1}_{\omega}} d m(\omega)<0 .
$$

Remark 2.16. Since the sequences $\left\{\omega \mapsto\left\|g_{\omega}^{(n)}\right\|_{\infty}\right\}_{n \in \mathbb{N}}$ and $\left\{\omega \mapsto \inf \mathcal{L}_{\omega}^{n} \mathbb{1}_{\omega}\right\}_{n \in \mathbb{N}}$ are submultiplicative and supermultiplicative, respectively, in view of Lemma 2.13 the subadditive ergodic theorem implies that (CP1) is equivalent to the assumption that there exist $N_{2}, N_{1} \in \mathbb{N}$ such that

$$
\frac{1}{N_{2}} \int_{\Omega} \log \left\|g_{\omega}^{\left(N_{2}\right)}\right\|_{\infty} d m(\omega)<\frac{1}{N_{1}} \int_{\Omega} \log \inf \mathcal{L}_{\omega}^{N_{1}} \mathbb{1}_{\omega} d m(\omega)
$$

If $\log \left\|g_{\omega}^{(n)}\right\|_{\infty} \in L_{m}^{1}(\Omega)$, then (CP1) is equivalent to (CP2) is equivalent to (2.13).

Remark 2.17. Note that in the deterministic setting our contracting potential assumption (CP1) implies the contracting potential assumption of [33], and is implied by the usual assumption that $\sup \varphi<P(\varphi)$, where $P(\varphi)$ denotes the topological pressure, see, for example, [15].

Definition 2.18. We will call the tuple $(\Omega, \sigma, m, I, T, \varphi)$ a random weighted covering system if it satisfies our assumptions (GP), (SP1)-(SP3), (RC), (M1)-(M6), and (CP1) and (CP2).

2.3. Main Results. Our main results are the following.

Theorem 2.19. Given a random weighted covering system $(\Omega, \sigma, m, I, T, \varphi)$, the following hold.

(1) There exists a unique random probability measure $\nu \in \mathcal{P}_{\Omega}(I)$ such that

$$
\nu_{\sigma(\omega)}\left(\mathcal{L}_{\omega} f\right)=\lambda_{\omega} \nu_{\omega}(f)
$$

for each $f \in \mathrm{BV}(I)$, where

$$
\lambda_{\omega}:=\nu_{\sigma(\omega)}\left(\mathcal{L}_{\omega} \mathbb{1}_{\omega}\right)
$$

Furthermore, we have that $\log \lambda_{\omega} \in L_{m}^{1}(\Omega)$.

(2) There exists a function $q \in \mathrm{BV}_{\Omega}(I)$ such that $\nu(q)=1$ and for $m$-a.e. $\omega \in \Omega$ we have

$$
\mathcal{L}_{\omega} q_{\omega}=\lambda_{\omega} q_{\sigma(\omega)}
$$

Moreover, $q$ is unique modulo $\nu$, and

$$
\lim _{|n| \rightarrow \infty} \frac{1}{|n|} \log \inf q_{\sigma^{n}(\omega)}=\lim _{|n| \rightarrow \infty} \frac{1}{|n|} \log \left\|q_{\sigma^{n}(\omega)}\right\|_{\infty}=0 .
$$

(3) The measure $\mu:=q \nu$ is a T-invariant and ergodic random probability measure supported on $\mathrm{J}$.

We also show that the operator cocycle is quasi-compact.

Theorem 2.20. With the same hypotheses as Theorem 2.19, for each $f \in \mathrm{BV}_{\Omega}^{l}(I)$ there exists a measurable function $\Omega \ni \omega \mapsto B_{f}(\omega) \in(0, \infty)$ and $\kappa \in(0,1)$ such that for m-a.e. $\omega \in \Omega$ and all $n \in \mathbb{N}$ we have

$$
\left\|\left(\lambda_{\omega}^{n}\right)^{-1} \mathcal{L}_{\omega}^{n} f_{\omega}-\nu_{\omega}\left(f_{\omega}\right) q_{\sigma^{n}(\omega)}\right\|_{\infty} \leq B_{f}(\omega)\left\|f_{\omega}\right\|_{\mathrm{BV}} \kappa^{n}
$$


and

$$
\left\|\left(\lambda_{\sigma^{-n}(\omega)}^{n}\right)^{-1} \mathcal{L}_{\sigma^{-n}(\omega)}^{n} f_{\sigma^{-n}(\omega)}-\nu_{\sigma^{-n}(\omega)}\left(f_{\sigma^{-n}(\omega)}\right) q_{\omega}\right\|_{\infty} \leq B_{f}(\omega)\left\|f_{\sigma^{-n}(\omega)}\right\|_{\mathrm{BV}} \kappa^{n} .
$$

From quasi-compactness we easily deduce the exponential decay of correlations.

Theorem 2.21. With the same hypotheses as Theorem 2.19, for every $h \in \mathrm{BV}_{\Omega}^{l}(I)$ and every $\varkappa \in(\kappa, 1)$, with $\kappa$ as in Theorem 2.20, there exists a measurable function $\Omega \ni \omega \mapsto$ $C_{h}(\omega) \in(0, \infty)$ such that for $m$-a.e. $\omega \in \Omega$, every $n \in \mathbb{N}$, and every $f \in L_{\mu}^{1}(\Omega \times I)$ we have

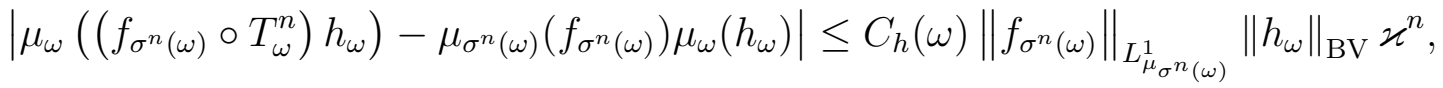

and

$$
\left|\mu_{\sigma^{-n}(\omega)}\left(\left(f_{\omega} \circ T_{\sigma^{-n}(\omega)}^{n}\right) h_{\sigma^{-n}(\omega)}\right)-\mu_{\omega}\left(f_{\omega}\right) \mu_{\sigma^{-n}(\omega)}\left(h_{\sigma^{-n}(\omega)}\right)\right| \leq C_{h}(\omega)\left\|f_{\omega}\right\|_{L_{\mu_{\omega}}^{1}}\left\|h_{\sigma^{-n}(\omega)}\right\|_{\mathrm{BV}} \varkappa^{n} .
$$

Theorem 2.20 is proven in Section 10, while Theorems 2.19 and 2.21 are proven in Section 11.

Let $\mathcal{P}_{T}(\Omega \times I) \subseteq \mathcal{P}_{\Omega}(I)$ denote the set of all $T$-invariant random probability measures on I. For $\eta \in \mathcal{P}_{T}(\Omega \times I)$ we denote the (fiberwise) conditional information of the partition $Z_{\omega}^{*}$ given $T_{\omega}^{-1} \mathscr{B}$, with respect to $\eta_{\omega}$, by $I_{\eta_{\omega}}$, which is given by

$$
I_{\eta_{\omega}}=I_{\eta_{\omega}}\left[Z_{\omega}^{*} \mid T_{\omega}^{-1} \mathscr{B}\right]:=-\log g_{\eta, \omega}
$$

where

$$
g_{\eta, \omega}:=\sum_{Z \in \mathcal{Z}_{\omega}^{*}} \mathbb{1}_{Z} E_{\eta_{\omega}}\left(\mathbb{1}_{Z} \mid T_{\omega}^{-1} \mathscr{B}\right)
$$

and $E_{\eta_{\omega}}\left(\mathbb{1}_{Z} \mid T_{\omega}^{-1} \mathscr{B}\right)$ denotes the conditional expectation of $\mathbb{1}_{Z}$ with respect to $\eta_{\omega}$ given the $\sigma$-algebra $T_{\omega}^{-1} \mathscr{B}$.

Definition 2.22. Given a contracting potential $\varphi: \Omega \times I \rightarrow \mathbb{R} \cup\{-\infty\}$ we define the expected pressure of $\varphi$ to be

$$
\mathcal{E} P(\varphi):=\int_{\Omega} \log \lambda_{\omega} d m(\omega)
$$

and we say that a random measure $\eta \in \mathcal{P}_{T}(\Omega \times I)$ is a relative equilibrium state if

$$
\mathcal{E} P(\varphi)=\int_{\Omega}\left(\int_{I} I_{\eta_{\omega}}+\varphi_{\omega} d \eta_{\omega}\right) d m(\omega)
$$

For countable partitions, it is possible that both the entropy and the integral of the potential could be infinite, which is why we state the following variational principle in terms of the conditional information as in [33].

Theorem 2.23. The T-invariant, ergodic random probability measure $\mu$ produced in Theorem 2.19 is the unique relative equilibrium state for $\varphi$, i.e.

$$
\mathcal{E} P(\varphi)=\int_{\Omega}\left(\int_{I} I_{\mu_{\omega}}+\varphi_{\omega} d \mu_{\omega}\right) d m(\omega) \geq \sup _{\eta \in \mathcal{P}_{T}(\Omega \times I)} \int_{\Omega}\left(\int_{I} I_{\eta_{\omega}}+\varphi_{\omega} d \eta_{\omega}\right) d m(\omega),
$$

where equality holds if and only if $\eta_{\omega}=\mu_{\omega}$ for $m$-a.e. $\omega \in \Omega$.

The proof of Theorem 2.23 is presented in Section 12. 
Remark 2.24. For $\eta \in \mathcal{P}_{T}(\Omega \times I)$ let $H_{\eta_{\omega}}\left(\mathcal{Z}_{\omega}^{*}\right)$ denote the entropy of the partition $Z_{\omega}^{*}$, which is given by

$$
H_{\eta_{\omega}}\left(Z_{\omega}^{*}\right):=-\sum_{Z \in Z_{\omega}^{*}} \eta_{\omega}(Z) \log \eta_{\omega}(Z)
$$

and let $h_{\eta}(T)$ denote the fiber entropy of $T$ with respect to $\eta$. Since $\mathcal{Z}_{\omega}^{*}$ is assumed to be generating, if $m\left(H_{\eta_{\omega}}\left(Z_{\omega}^{*}\right)\right)<\infty$, then we have

$$
h_{\eta}(T)=\int_{\Omega} H_{\eta_{\omega}}\left(\mathcal{Z}_{\omega}^{*} \mid T_{\omega}^{-1} \mathscr{B}\right) d m(\omega)=\int_{\Omega} \int_{I} I_{\eta_{\omega}} d \eta_{\omega} d m(\omega) .
$$

Thus, we note that in the case that $m\left(H_{\mu_{\omega}}\left(Z_{\omega}^{*}\right)\right)<\infty$, Theorem 2.23 reduces to the usual variational principle, that is

$$
\mathcal{E} P(\varphi)=h_{\mu}(T)+\int_{\Omega} \int_{I} \varphi_{\omega} d \mu_{\omega} d m(\omega) \geq \sup _{\substack{n \in \mathcal{P}_{T}(\Omega \times I) \\ m\left(H_{\eta_{\omega}}\left(\mathcal{Z}_{\omega}^{*}\right)\right)<\infty}} h_{\eta}(T)+\int_{\Omega} \int_{I} \varphi_{\omega} d \eta_{\omega} d m(\omega) .
$$

See [6] for details. In particular, this holds if $z_{\omega}^{*}$ is finite for $m$-a.e. $\omega \in \Omega$.

In what follows, we will assume throughout that we are working with a random weighted covering system $(\Omega, \sigma, m, I, T, \varphi)$.

\section{Existence of a Conformal Family of Measures}

In this section we prove the existence of a conformal family of measures. We obtain these measures, as is often the case, via fixed point methods, see for example [25] or [39, Chapter 3], for the deterministic and random settings respectively. In the continuous random setting, one considers the transfer operator $\mathcal{L}_{\omega}$ acting on the space of continuous bounded real-valued functions, and then applies the Schauder-Tichonov Fixed Point Theorem to the map $\nu_{\sigma(\omega)} \mapsto \mathcal{L}_{\omega}^{*} \nu_{\sigma(\omega)} / \mathcal{L}_{\omega}^{*} \nu\left(\mathbb{1}_{\sigma(\omega)}\right)$, where $\mathcal{L}_{\omega}^{*}$ is the dual operator, to obtain a functional $\nu_{\omega}$ such that $\mathcal{L}_{\omega}^{*} \nu_{\sigma(\omega)}=\lambda_{\omega} \nu_{\omega}$ where $\lambda_{\omega}=\nu_{\sigma(\omega)}\left(\mathcal{L}_{\omega} \mathbb{1}_{\omega}\right)$. In this setting, the functional $\nu_{\omega}$ can be uniquely identified with a Borel probability measure via the Riesz Representation Theorem. However, to deal with discontinuities we work with BV functions rather than continuous functions, so our strategy differs from that of [25] or [39] in that our functional $\Lambda_{\omega}$, which we obtain via the Schauder-Tichonov Fixed Point Theorem, must first be extended to the space of continuous functions so that we may identify $\Lambda_{\omega}$ with a measure $\nu_{\omega}$ via Riesz's Theorem. We must then show that the measure $\nu_{\omega}$ is equal to the functional $\Lambda_{\omega}$ when restricted to BV functions. After obtaining such a family of measures $\left(\nu_{\omega}\right)_{\omega \in \Omega}$ it is natural to then ask whether this family is a random probability measure as in Definition 2.5. As we do not currently have the tools to deal with the question of $m$-measurability, we will leave this task for later, specifically Section 9. For now, we simply prove that a conformal family of measures exists.

The main result of this section is the following.

Proposition 3.1. There exists a family $\left(\nu_{\omega}\right)_{\omega \in \Omega}$ of non-atomic Borel probability measures, i.e. $\nu_{\omega} \in \mathcal{P}(I)$, with

$$
\int_{I} \mathcal{L}_{\omega} f d \nu_{\sigma(\omega)}=\lambda_{\omega} \int_{I} f d \nu_{\omega}
$$

for each $\omega \in \Omega$ and $f \in L_{\nu_{\omega}}^{1}(I)$, where

$$
\lambda_{\omega}:=\nu_{\sigma(\omega)}\left(\mathcal{L}_{\omega} \mathbb{1}_{\omega}\right)
$$


such that

(i) $\nu_{\omega}\left(X_{\omega}\right)=1$,

(ii) $\nu_{\omega}(J)>0$

for each non-degenerate interval $J \subseteq I$.

Proof. For each $\omega \in \Omega$ let $\mathcal{M}_{\omega}^{1}$ denote the set of all positive linear functionals $\Gamma \in \mathrm{BV}^{*}\left(X_{\omega}\right)$ such that $\Gamma\left(\mathbb{1}_{\omega}\right)=1$. We begin by noting that $\mathcal{M}_{\omega}^{1}$ is a convex and weak*-closed subset of the norm unit ball in $\mathrm{BV}^{*}\left(X_{\omega}\right)$, which is weak*-compact by the Banach-Alaoglu Theorem. Thus we have that the set

$$
\mathcal{M}^{1}=\left\{\Gamma=\left(\Gamma_{\omega}\right)_{\omega \in \Omega}: \Gamma_{\omega} \in \mathcal{M}_{\omega}^{1}\right\}
$$

is a compact and convex subset of a locally convex topological vector space, namely the product $\prod_{\omega \in \Omega} \mathrm{BV}^{*}\left(X_{\omega}\right)$. Define the map $\Psi=\left(\Psi_{\omega}\right)_{\omega \in \Omega}: \mathcal{M}^{1} \rightarrow \mathcal{M}^{1}$ by

$$
\Psi_{\omega}\left(\Gamma_{\sigma(\omega)}\right)=\frac{\mathcal{L}_{\omega}^{*} \Gamma_{\sigma(\omega)}}{\mathcal{L}_{\omega}^{*} \Gamma_{\sigma(\omega)}\left(\mathbb{1}_{\omega}\right)}
$$

Clearly $\Psi_{\omega}$ is weak ${ }^{*}$-continuous, and thus $\Psi$ is continuous with respect to coordinate convergence, and thus $\Psi$ is continuous with respect to the product topology. Applying the Schauder-Tichonov Theorem produces a $\Lambda \in \mathcal{M}^{1}$ which is a fixed point of $\Psi$. In other words, we have that there exists $\Lambda=\left(\Lambda_{\omega}\right)_{\omega \in \Omega}$ such that

$$
\mathcal{L}_{\omega}^{*} \Lambda_{\sigma(\omega)}(f)=\lambda_{\omega} \Lambda_{\omega}(f)
$$

for all $f \in \mathrm{BV}\left(X_{\omega}\right)$, where

$$
\lambda_{\omega}:=\Lambda_{\sigma(\omega)}\left(\mathcal{L}_{\omega} \mathbb{1}_{\omega}\right)
$$

In particular, iteration of (3.2) gives that

$$
\Lambda_{\sigma^{n}(\omega)}\left(\mathcal{L}_{\omega}^{n} f\right)=\lambda_{\omega}^{n} \Lambda_{\omega}(f)
$$

for each $n \in \mathbb{N}$ and all $f \in \mathrm{BV}\left(X_{\omega}\right)$, where

$$
\lambda_{\omega}^{n}:=\Lambda_{\sigma^{n}(\omega)}\left(\mathcal{L}_{\omega}^{n} \mathbb{1}_{\omega}\right)=\prod_{j=0}^{n-1} \lambda_{\sigma^{j}(\omega)} .
$$

Note that we must have

$$
\lambda_{\omega}>0 \text {. }
$$

To see this we note that the random covering hypothesis (RC) implies the existence of some $M_{\omega}(I)$ such that for all $n \geq M_{\omega}(I)$, inf $\mathcal{L}_{\omega}^{n} \mathbb{1}_{\omega}>0$, which implies that $\lambda_{\omega}^{n}>0$, which, in light of (3.4), in turn implies that $\lambda_{\omega}>0$.

We can then extend $\Lambda_{\omega}$ to $\mathrm{BV}(I)$ by

$$
\Lambda_{\omega}(h):=\Lambda_{\omega}\left(\left.h\right|_{X_{\omega}}\right), \quad h \in \mathrm{BV}(I) .
$$

By the compactness of $I$, each continuous function $f \in \mathcal{C}(I)$ can be approximated in $\mathcal{C}(I)$ by a sequence of functions in BV $(I)$. Thus, each random continuous function can be approximated uniformly by random BV functions, which means that for each $\omega \in \Omega$ we can extend $\Lambda_{\omega}$ to $\mathcal{C}(I)$. By the Riesz Theorem we can then identify the functional $\Lambda_{\omega}$ with a random Borel probability measure $\nu_{\omega}$ on $I$ which agrees with $\Lambda_{\omega}$ on $\mathcal{C}(I)$.

Working towards showing that $\nu_{\omega}$ agrees with $\Lambda_{\omega}$ on $\mathrm{BV}\left(X_{\omega}\right)$, we first show that $\nu_{\omega}(J)=$ $\Lambda_{\omega}\left(\mathbb{1}_{J}\right)>0$ for all non-degenerate intervals $J \subseteq I$. 
Claim 3.1.1. For all non-degenerate intervals $J \subseteq I$ we have

$$
\nu_{\omega}(J)=\Lambda_{\omega}\left(\mathbb{1}_{J}\right)>0 .
$$

Proof. We first prove that $\Lambda_{\omega}\left(\mathbb{1}_{J}\right)>0$ for all non-degenerate intervals $J \subseteq I$. To that end, we let $M=M_{\omega}(J)$ and $C=C_{\omega, M_{\omega}(J)}(J)$ be the constants coming from our random covering assumption $(\mathrm{RC})$. Then we note that (3.3) gives

$$
\lambda_{\omega}^{M} \Lambda_{\omega}\left(\mathbb{1}_{J}\right)=\Lambda_{\sigma^{M}(\omega)}\left(\mathcal{L}_{\omega}^{M} \mathbb{1}_{J}\right) \geq C>0,
$$

and hence,

$$
\Lambda_{\omega}\left(\mathbb{1}_{J}\right) \geq \frac{C}{\lambda_{\omega}^{M}}>0
$$

Now suppose that $\bar{J}=[a, b]$ and let $\varepsilon>0$. In light of our contracting potential assumption (CP1), choose $n \in \mathbb{N}$ so large that

$$
\frac{\left\|g_{\omega}^{(n)}\right\|_{\infty}}{\inf \mathcal{L}_{\omega}^{n} \mathbb{1}_{\omega}}<\frac{\varepsilon}{4}
$$

Note that since $T_{\omega}^{n}$ is injective on each element of $\mathcal{Z}_{\omega}^{(n)}$, we must have that

$$
\mathcal{L}_{\omega}^{n} \mathbb{1}_{Z} \leq\left\|g_{\omega}^{(n)}\right\|_{\infty}
$$

for each $Z \in \mathcal{Z}_{\omega}^{(n)}$. Thus, in light of (3.3), (3.7), and (3.8), for all $n \in \mathbb{N}$ and all $Z \in \mathcal{Z}_{\omega}^{(n)}$ we have that

$$
\Lambda_{\omega}\left(\mathbb{1}_{Z}\right)=\left(\lambda_{\omega}^{n}\right)^{-1} \Lambda_{\sigma^{n}(\omega)}\left(\mathcal{L}_{\omega}^{n} \mathbb{1}_{Z}\right) \leq \frac{\left\|g_{\omega}^{(n)}\right\|_{\infty}}{\inf \mathcal{L}_{\omega}^{n} \mathbb{1}_{\omega}}<\frac{\varepsilon}{4} .
$$

We now claim that there exist open intervals $V_{a}, V_{b} \subseteq I$ containing $a$ and $b$, respectively, such that $\Lambda_{\omega}\left(V_{a}\right)+\Lambda_{\omega}\left(V_{b}\right)<\varepsilon$. To see this, we first construct $V_{a}$ by noting that (3.9) taken together with the fact that $\sum_{Z \in \mathcal{Z}_{\omega}^{(n)}} \Lambda_{\omega}\left(\mathbb{1}_{Z}\right)=1$ allows us to find a (not necessarily finite) sub-collection $Q_{a} \subseteq Z_{\omega}^{(n)}$ such that

(1) $\sum_{Z \in Q_{a}} \Lambda_{\omega}\left(\mathbb{1}_{Z}\right)<\frac{\varepsilon}{2}$

(2) there exist $\alpha_{a}, \beta_{a} \in X_{\omega}{ }^{4}$ with $\alpha_{a} \leq a \leq \beta_{a}$ such that

$$
\alpha_{a}, \beta_{a} \in \operatorname{Int}\left(\bigcup_{Z \in Q_{a}} Z\right) .
$$

Finally, take $V_{a}=\operatorname{Int}\left(\bigcup_{Z \in Q_{a}} Z\right)$. The construction of $V_{b}$ proceeds in a similar manner. Let $H=J \cup V_{a} \cup V_{b}$ and let $f \in \mathrm{BV}(I) \cap \mathcal{C}(I)$ such that

$$
\mathbb{1}_{J} \leq\left. f\right|_{H} \leq 1
$$

and $\left.f\right|_{H^{c}}=0 .{ }^{5}$ Thus, we have

$$
\nu_{\omega}\left(\mathbb{1}_{J}\right) \leq \Lambda_{\omega}(f) \leq \Lambda_{\omega}\left(\mathbb{1}_{J}\right)+\Lambda_{\omega}\left(\mathbb{1}_{V_{a}}\right)+\Lambda_{\omega}\left(\mathbb{1}_{V_{b}}\right)<\Lambda_{\omega}\left(\mathbb{1}_{J}\right)+\varepsilon .
$$

\footnotetext{
${ }^{4}$ If $a \in X_{\omega}$ we may take $\alpha_{a}=a=\beta_{a}$.

${ }^{5}$ One could prove the existence of such a function in a similar manner to Urysohn's Lemma to show that for $x<y$ there exists a continuous, increasing function (hence BV) from $[x, y]$ onto $[0,1]$.
} 
As $\varepsilon>0$ was arbitrary, we must have that $\nu_{\omega}(J) \leq \Lambda_{\omega}\left(\mathbb{1}_{J}\right)$ for all intervals $J \subseteq I$. To see the opposite inequality we simply note that for any disjoint intervals $A, B \subseteq I$ with $A \cup B=I$ we have

$$
1=\nu_{\omega}(A)+\nu_{\omega}(B) \leq \Lambda_{\omega}\left(\mathbb{1}_{A}\right)+\Lambda_{\omega}\left(\mathbb{1}_{B}\right)=1 .
$$

Thus, taking $A=J$ and $B=J^{c}$ in the above equation, we have must in fact have $\Lambda_{\omega}\left(\mathbb{1}_{J}\right)=$ $1-\nu_{\omega}\left(J^{c}\right)=\nu_{\omega}(J)$ for all intervals $J \subseteq I$.

Note that we have just shown in the proof of Claim 3.1.1 that given any $a \in X_{\omega}$, any $\varepsilon>0$, and sufficiently large $n \in \mathbb{N}$ we can find a collection $\mathcal{Q} \subseteq z_{\omega}^{(n)}$ such that

(1) $a \in \cup_{Z \in Q} Z=: V$,

(2) $0<\nu_{\omega}(V)<\varepsilon$.

In conjunction with the fact that $\nu_{\omega}\left(X_{\omega}\right)=\Lambda_{\omega}\left(\mathbb{1}_{\omega}\right)=1$, we see that $\nu_{\omega}$ has no atoms, and in particular that the singular set has measure zero, i.e.

$$
\nu_{\omega}\left(\mathcal{S}_{\omega}\right)=\nu_{\omega}\left(I \backslash X_{\omega}\right)=0 .
$$

We are now able to show that $\nu_{\omega}$ agrees with $\Lambda_{\omega}$ on $\mathrm{BV}(I)$.

Claim 3.1.2. For all $f \in \mathrm{BV}(I)$ we have

$$
\nu_{\omega}(f)=\Lambda_{\omega}(f)
$$

Proof. Let $\varepsilon>0$. Since the jump discontinuities of $f$ can be larger than $\varepsilon$ on only a finite set $D_{\omega, \varepsilon}$, we can find a piecewise continuous function $f_{\varepsilon}: I \rightarrow \mathbb{R}$ such that $\left\|f-f_{\varepsilon}\right\|_{\infty}<\varepsilon$. Since $f_{\varepsilon}$ is piecewise continuous we have that $\nu_{\omega}\left(f_{\varepsilon}\right)=\Lambda_{\omega}\left(f_{\varepsilon}\right)$, and hence

$$
\begin{aligned}
\left|\nu_{\omega}(f)-\Lambda_{\omega}(f)\right| & \leq\left|\nu_{\omega}\left(f-f_{\varepsilon}\right)\right|+\left|\nu_{\omega}\left(f_{\varepsilon}\right)-\Lambda_{\omega}\left(f_{\varepsilon}\right)\right|+\left|\Lambda_{\omega}\left(f-f_{\varepsilon}\right)\right| \\
& \leq 2\left\|f-f_{\varepsilon}\right\|_{\infty}<2 \varepsilon .
\end{aligned}
$$

As $\varepsilon>0$ is arbitrary, we must have that $\nu_{\omega}(f)=\Lambda_{\omega}(f)$.

An immediate consequence of Claim 3.1.2 is that

$$
\nu_{\sigma(\omega)}\left(\mathcal{L}_{\omega} f\right)=\Lambda_{\sigma(\omega)}\left(\mathcal{L}_{\omega} f\right)=\lambda_{\omega} \Lambda_{\omega}(f)=\lambda_{\omega} \nu_{\omega}(f)
$$

for all $f \in L_{\nu_{\omega}}^{1}(I)$, which finishes the proof of Proposition 3.1.

We can immediately see, cf. [17], that the conformality of the family $\left(\nu_{\omega}\right)_{\omega \in \Omega}$ produced in Proposition 3.1 can be characterized equivalently as: for each $n \geq 1$ and each set $A$ on which $\left.T_{\omega}^{n}\right|_{A}$ is one-to-one we have

$$
\nu_{\sigma^{n}(\omega)}\left(T_{\omega}^{n}(A)\right)=\lambda_{\omega}^{n} \int_{A} e^{-S_{n, T}\left(\varphi_{\omega}\right)} d \nu_{\omega}
$$

where

$$
\lambda_{\omega}^{n}=\nu_{\sigma^{n}(\omega)}\left(\mathcal{L}_{\omega}^{n_{\omega}} \mathbb{1}_{\omega}\right)=\prod_{j=0}^{n-1} \lambda_{\sigma^{j}(\omega)} .
$$

In particular, this gives that for each $n \geq 1$ and each $Z \in Z_{\omega}^{(n)}$ we have

$$
\nu_{\sigma^{n}(\omega)}\left(T_{\omega}^{n}(Z)\right)=\lambda_{\omega}^{n} \int_{Z} e^{-S_{n, T}\left(\varphi_{\omega}\right)} d \nu_{\omega} .
$$


Definition 3.2. We define the normalized operator (for any $n \geq 1) \tilde{\mathcal{L}}_{\omega}^{n}: \mathcal{B}\left(X_{\omega}\right) \rightarrow \mathcal{B}\left(X_{\sigma^{n}(\omega)}\right)$ by

$$
\tilde{\mathcal{L}}_{\omega}^{n}(f):=\left(\lambda_{\omega}^{n}\right)^{-1} \mathcal{L}_{\omega}^{n} f, \quad f \in \mathcal{B}\left(X_{\omega}\right) .
$$

In light of (3.10) we immediately see that this normalized operator satisfies the following:

$$
\nu_{\sigma^{n}(\omega)}\left(\tilde{\mathcal{L}}_{\omega}^{n} f\right)=\nu_{\omega}(f), \quad f \in L_{\nu_{\omega}}^{1}(I) .
$$

\section{LASOTA-Yorke INEQUALITIES}

In this section we prove Lasota-Yorke inequalities. We first prove a random version of the Lasota-Yorke inequality appearing in [33]. However, the coefficients produced in this inequality are highly $\omega$-dependent and may grow too much with further iteration. To rectify this, we then follow [10] to prove a more refined Lasota-Yorke inequality, Proposition 4.9, with more manageable $\omega$-dependent coefficients.

The following Lasota-Yorke type inequality gives a useful bound on the variation of the image of the transfer operator, which will be crucial in the sequel, and in fact implies that

$$
\mathcal{L}_{\omega}^{n}\left(\mathrm{BV}\left(X_{\omega}\right)\right) \subseteq \mathrm{BV}\left(X_{\sigma^{n}(\omega)}\right)
$$

for all $n \in \mathbb{N}$ and $\omega \in \Omega$.

Lemma 4.1. Suppose that $\hat{\alpha} \geq 0$ and $\hat{\gamma} \geq 1$ and that for each $\omega \in \Omega$ and $n \in \mathbb{N}$ there exists a partition $\mathcal{P}_{\omega, n}(\hat{\alpha}, \hat{\gamma})$ which satisfies $(\mathrm{P} 1)$ and $(\mathrm{P} 2)$. Then for all $\omega \in \Omega$, all $f \in \mathrm{BV}\left(X_{\omega}\right)$, and all $n \in \mathbb{N}$ there exist positive, measurable constants $A_{\omega}^{(n)}$ and $B_{\omega}^{(n)}$ such that

$$
\operatorname{var}\left(\mathcal{L}_{\omega}^{n} f\right) \leq A_{\omega}^{(n)}\left(\operatorname{var}(f)+B_{\omega}^{(n)} \nu_{\omega}(|f|)\right)
$$

where

$$
A_{\omega}^{(n)}:=(\hat{\alpha}+2 \hat{\gamma}+1)\left\|g_{\omega}^{(n)}\right\|_{\infty} \quad \text { and } \quad B_{\omega}^{(n)}:=\frac{(\hat{\alpha}+2 \hat{\gamma})\left\|\mathcal{L}_{\omega}^{M_{\omega, n}} \mathbb{1}_{\omega}\right\|_{\infty}}{\inf _{J\left(P_{\omega, n}\right)} g_{\omega}^{\left(M_{\omega, n}\right)}}
$$

where $P_{\omega, n}$ is some element of the finite partition $\mathcal{P}_{\omega, n}(\hat{\alpha}, \hat{\gamma}), J\left(P_{\omega, n}\right)$ is the monotonicity partition element coming from (2.10), and $M_{\omega, n}=M_{\omega}\left(J\left(P_{\omega, n}\right)\right)$.

Proof. Recall that Lemma 2.4 gives that $g_{\omega}^{(n)} \in \mathrm{BV}\left(X_{\omega}\right)$ for each $n \in \mathbb{N}$ and $\omega \in \Omega$. Thus, for $f \in \mathrm{BV}\left(X_{\omega}\right)$ and $n \geq 0$ we have

$$
\begin{aligned}
\operatorname{var}\left(\mathcal{L}_{\omega}^{n} f\right) & \leq \operatorname{var}\left(\sum_{Z \in \mathcal{Z}_{\omega}^{(n)}} \mathbb{1}_{T_{\omega}^{n}(Z)}\left(\left(g_{\omega}^{(n)} f\right) \circ T_{\omega, Z}^{-n}\right)\right) \\
& \leq \sum_{Z \in \mathcal{Z}_{\omega}^{(n)}} \operatorname{var}\left(\mathbb{1}_{T_{\omega}^{n}(Z)}\left(\left(g_{\omega}^{(n)} f\right) \circ T_{\omega, Z}^{-n}\right)\right) \\
& \leq \sum_{Z \in \mathcal{Z}_{\omega}^{(n)}} \operatorname{var}\left(g_{\omega}^{(n)} f \mathbb{1}_{Z}\right) \\
& \leq \sum_{Z \in \mathcal{Z}_{\omega}^{(n)}}\left(\operatorname{var}_{Z}\left(g_{\omega}^{(n)} f\right)+2\left|\sup _{Z} g_{\omega}^{(n)} f\right|\right)
\end{aligned}
$$




$$
\leq \operatorname{var}\left(g_{\omega}^{(n)} f\right)+2 \sum_{Z \in \mathcal{Z}_{\omega}^{(n)}}\left|\sup _{Z} g_{\omega}^{(n)} f\right| .
$$

Thus, using (4.1) and (P1)-(P2), we get

$$
\begin{aligned}
\operatorname{var}\left(\mathcal{L}_{\omega}^{n} f\right) & \leq \sum_{P \in \mathcal{P}_{\omega, n}(\hat{\alpha}, \hat{\gamma})}\left(\operatorname{var}_{P}\left(g_{\omega}^{(n)} f\right)+2 \sum_{\substack{Z \in \mathcal{Z}_{\omega}^{(n)} \\
Z \cap P \neq \emptyset}}\left|\sup _{Z \cap P} g_{\omega}^{(n)} f\right|\right) \\
& \leq \sum_{P \in \mathcal{P}_{\omega, n}(\hat{\alpha}, \hat{\gamma})}\left(\left\|g_{\omega}^{(n)}\right\|_{\infty} \operatorname{var}_{P}(f)+\left\|f \mathbb{1}_{P}\right\|_{\infty}\left(\operatorname{var}_{P}\left(g_{\omega}^{(n)}\right)+2 \sum_{\substack{Z \in \mathcal{Z}_{\omega}^{(n)} \\
Z \cap P \neq \emptyset}} \sup _{Z \rightarrow \infty} g_{\omega}^{(n)}\right)\right) \\
& \leq \sum_{P \in \mathcal{P}_{\omega, n}(\hat{\alpha}, \hat{\gamma})}\left(\left\|g_{\omega}^{(n)}\right\|_{\infty} \operatorname{var}_{P}(f)+(\hat{\alpha}+2 \hat{\gamma})\left\|g_{\omega}^{(n)}\right\|_{\infty}\left\|\left.f\right|_{P}\right\|_{\infty}\right) \\
& \leq \sum_{P \in \mathcal{P}_{\omega, n}(\hat{\alpha}, \hat{\gamma})}\left((\hat{\alpha}+2 \hat{\gamma}+1)\left\|g_{\omega}^{(n)}\right\|_{\infty} \operatorname{var}_{P}(f)+(\hat{\alpha}+2 \hat{\gamma})\left\|g_{\omega}^{(n)}\right\|_{\infty} \inf |f|_{P} \mid\right) \\
& \leq \sum_{P \in \mathcal{P}_{\omega, n}(\hat{\alpha}, \hat{\gamma})}\left((\hat{\alpha}+2 \hat{\gamma}+1)\left\|g_{\omega}^{(n)}\right\|_{\infty} \operatorname{var}_{P}(f)+(\hat{\alpha}+2 \hat{\gamma})\left\|g_{\omega}^{(n)}\right\|_{\infty} \frac{\nu_{\omega}\left(|f|_{P} \mid\right)}{\nu_{\omega}(P)}\right) .
\end{aligned}
$$

Note that Claim 3.1.1 from the proof of Proposition 3.1 gives that $\nu_{\omega}(P)>0$ for all $P \in$ $\mathcal{P}_{\omega, n}(\hat{\alpha}, \hat{\gamma})$. Recall that since $\mathcal{Z}_{\omega}^{(1)}$ is generating, see $(\mathrm{GP})$, for each $P \in \mathcal{P}_{\omega, n}(\hat{\alpha}, \hat{\gamma})$ there exists a least number $N_{\omega, n}(P) \in \mathbb{N}$ and a measurable choice

$$
J(P) \in \mathcal{Z}_{\omega}^{\left(N_{\omega, n}(P)\right)}
$$

with $J(P) \subseteq P$. In light of (SP1) we have

$$
\inf \mathcal{L}_{\omega}^{M_{\omega}(J(P))} \mathbb{1}_{P} \geq \inf _{J(P)} g_{\omega}^{M_{\omega}(J(P))}>0
$$

for each $P \in \mathcal{P}_{\omega, n}(\hat{\alpha}, \hat{\gamma})$, and thus for each $n \in \mathbb{N}$ and each $P \in \mathcal{P}_{\omega, n}(\hat{\alpha}, \hat{\gamma})$ we denote

$$
\hat{B}_{\omega}^{(n)}(P):=\frac{\left\|\mathcal{L}_{\omega}^{M_{\omega}(J(P))} \mathbb{1}_{\omega}\right\|_{\infty}}{\inf _{J(P)} g_{\omega}^{\left(M_{\omega}(J(P))\right)}}
$$

and let $P_{\omega, n}$ be the element of $\mathcal{P}_{\omega, n}(\hat{\alpha}, \hat{\gamma})$ which maximizes this quantity, i.e.

$$
\hat{B}_{\omega}^{(n)}\left(P_{\omega, n}\right):=\max _{P \in \mathcal{P}_{\omega}, n} \hat{B}_{\omega}^{(n)}(P) .
$$

Then for each $P \in \mathcal{P}_{\omega, n}(\hat{\alpha}, \hat{\gamma})$ we have

$$
\begin{aligned}
\nu_{\omega}\left(\mathbb{1}_{P}\right) & =\nu_{\sigma^{M_{\omega}(J(P))}(\omega)}\left(\tilde{\mathcal{L}}_{\omega}^{M_{\omega}(J(P))} \mathbb{1}_{P}\right) \geq\left(\lambda_{\omega}^{M_{\omega}(J(P))}\right)^{-1} \inf _{J(P)} g_{\omega}^{\left(M_{\omega}(J(P))\right)} \\
& \geq\left(\hat{B}_{\omega}^{(n)}(P)\right)^{-1} \geq\left(\hat{B}_{\omega}^{(n)}\left(P_{\omega, n}\right)\right)^{-1} .
\end{aligned}
$$

Inserting (4.3) into (4.2), we have

$$
\operatorname{var}\left(\mathcal{L}_{\omega}^{n} f\right) \leq A_{\omega}^{(n)}\left(\operatorname{var}(f)+B_{\omega}^{(n)} \nu_{\omega}(|f|)\right),
$$


where

$$
M_{\omega, n}:=M_{\omega}\left(J\left(P_{\omega, n}\right)\right)
$$

denotes the covering time of $J\left(P_{\omega, n}\right)$ and

$$
A_{\omega}^{(n)}:=(\hat{\alpha}+2 \hat{\gamma}+1)\left\|g_{\omega}^{(n)}\right\|_{\infty} \quad \text { and } \quad B_{\omega}^{(n)}:=\frac{(\hat{\alpha}+2 \hat{\gamma})\left\|\mathcal{L}_{\omega}^{M_{\omega, n}} \mathbb{1}_{\omega}\right\|_{\infty}}{\inf _{J\left(P_{\omega, n}\right)} g_{\omega}^{\left(M_{\omega, n}\right)}}
$$

which finishes the proof.

Define the random constants

$$
Q_{\omega}^{(n)}:=\frac{A_{\omega}^{(n)}}{\inf \mathcal{L}_{\omega}^{n} \mathbb{1}_{\omega}} \quad \text { and } \quad K_{\omega}^{(n)}:=Q_{\omega}^{(n)} B_{\omega}^{(n)} .
$$

In light of our contracting potential assumption (CP1) we see that $Q_{\omega}^{(n)} \rightarrow 0$ exponentially quickly for each $\omega \in \Omega$.

Remark 4.2. In order to achieve the exponential decay of the random constants $Q_{\omega}^{(n)}$ it would suffice to replace the fixed numbers $\hat{\alpha} \geq 0$ and $\hat{\gamma} \geq 1$ with increasing polynomials $\hat{\alpha}(n), \hat{\gamma}(n)$.

The following proposition now follows from Lemma 2.13 and assumptions (M5) and (M6).

Proposition 4.3. For each $n \in \mathbb{N}, \log ^{+} Q_{\omega}^{(n)}, \log K_{\omega}^{(n)} \in L_{m}^{1}(\Omega)$

Now, considering the normalized operator, we arrive at the following immediate corollary.

Corollary 4.4. For all $\omega \in \Omega$, all $f \in \mathrm{BV}\left(X_{\omega}\right)$, and all $n \in \mathbb{N}$ we have

$$
\operatorname{var}\left(\tilde{\mathcal{L}}_{\omega}^{n} f\right) \leq Q_{\omega}^{(n)} \operatorname{var}(f)+K_{\omega}^{(n)} \nu_{\omega}(|f|) .
$$

Definition 4.5. In view of our contracting potential assumption (CP2) and the submultiplicativity of $\left\|g_{\omega}^{(n)}\right\|_{\infty} / \inf \mathcal{L}_{\omega}^{n} \mathbb{1}_{\omega}$, we let $N_{*} \in \mathbb{N}$ be the minimum integer $n \geq 1$ such that

$$
-\infty<\int_{\Omega} \log Q_{\omega}^{(n)} d m(\omega)<0
$$

and we define the number

$$
\xi:=-\frac{1}{N_{*}} \int_{\Omega} \log Q_{\omega}^{\left(N_{*}\right)} d m(\omega) .
$$

In light of Corollary 4.4 we may now find an appropriate upper bound for the BV norm of the normalized transfer operator.

Lemma 4.6. There exists a measurable function $\omega \mapsto L_{\omega} \in(0, \infty)$ with $\log L_{\omega} \in L_{m}^{1}(\Omega)$ such that for all $f \in \mathrm{BV}(I)$ and each $1 \leq n \leq N_{*}$ we have

$$
\left\|\tilde{\mathcal{L}}_{\omega}^{n} f\right\|_{\mathrm{BV}} \leq L_{\omega}^{n}\left(\operatorname{var}(f)+\nu_{\omega}(|f|)\right)
$$

where

$$
L_{\omega}^{n}=L_{\omega} L_{\sigma(\omega)} \cdots L_{\sigma^{n-1}(\omega)} \geq 55^{n} .
$$


Proof. Corollary 4.4 and (3.10) give

$$
\begin{aligned}
\left\|\tilde{\mathcal{L}}_{\omega}^{n} f\right\|_{\mathrm{BV}} & =\operatorname{var}\left(\tilde{\mathcal{L}}_{\omega}^{n} f\right)+\left\|\tilde{\mathcal{L}}_{\omega}^{n} f\right\|_{\infty} \leq 2 \operatorname{var}\left(\tilde{\mathcal{L}}_{\omega}^{n} f\right)+\nu_{\sigma^{n}(\omega)}\left(\tilde{\mathcal{L}}_{\omega}^{n} f\right) \\
& \leq 2\left(Q_{\omega}^{(n)} \operatorname{var}(f)+K_{\omega}^{(n)} \nu_{\omega}(|f|)\right)+\nu_{\omega}(|f|) \\
& \leq 2 Q_{\omega}^{(n)} \operatorname{var}(f)+\left(2 K_{\omega}^{(n)}+1\right) \nu_{\omega}(|f|) .
\end{aligned}
$$

Now, set

$$
\tilde{L}_{\omega}^{(n)}:=\max \left\{55,2 Q_{\omega}^{(n)}, 2 K_{\omega}^{(n)}+1\right\}^{6} .
$$

Finally, setting

$$
L_{\omega}:=\max \left\{\tilde{L}_{\omega}^{(j)}: 1 \leq j \leq N_{*}\right\}
$$

and

$$
L_{\omega}^{n}:=\prod_{j=0}^{n-1} L_{\sigma^{j}(\omega)}
$$

for all $n \geq 1$ suffices. The log-integrability of $L_{\omega}^{n}$ follows from Proposition 4.3.

Definition 4.7. In light of Lemma 4.6 we now define the number

$$
\rho:=\frac{1}{N_{*}} \int_{\Omega} \log L_{\omega}^{N_{*}} d m(\omega) .
$$

Remark 4.8. We would like to note that the hypotheses (M5) and (M6) may be difficult to check for examples. In the sequel we will only need that these conditions hold for $n=N_{*}$. Thus, we will actually prove our results under the following weaker assumptions:

$\left(\mathrm{M} 5{ }^{\prime}\right) \min _{P \in \mathcal{P}_{\omega, N_{*}}} \log \inf _{J(P)} g_{\omega}^{M_{\omega}(J(P))} \in L_{m}^{1}(\Omega)$,

$\left(\mathrm{M} 6{ }^{\prime}\right) \max _{P \in \mathcal{P}_{\omega, N_{*}}} \log \left\|\mathcal{L}_{\omega}^{M_{\omega}(J(P))} \mathbb{1}_{\omega}\right\|_{\infty} \in L_{m}^{1}(\Omega)$.

Of course this means that we need only to consider (P1) and (P2) for $n=N_{*}$ as well.

The constants $K_{\omega}^{(n)}$ from Corollary 4.4 grow to infinity with $n$, making them difficult to use. We therefore now follow Buzzi [10, Lemma 2.1] to prove the main result of this section, a similar, but more useful Lasota-Yorke type inequality.

Proposition 4.9. For each $\varepsilon>0$ there exists a measurable, $m$-a.e. finite function $C_{\varepsilon}(\omega)>0$ such that for m-a.e. $\omega \in \Omega$, each $f \in \mathrm{BV}\left(X_{\sigma^{-n}(\omega)}\right)$, and all $n \in \mathbb{N}$ we have

$$
\operatorname{var}\left(\tilde{\mathcal{L}}_{\sigma^{-n}(\omega)}^{n} f\right) \leq C_{\varepsilon}(\omega) e^{-(\xi-\varepsilon) n} \operatorname{var}(f)+C_{\varepsilon}(\omega) \nu_{\sigma^{-n}(\omega)}(|f|) .
$$

Proof. We begin by noting that since $\sigma$ is ergodic and writing

$$
\sum_{k=0}^{n N_{*}-1} \psi \circ \sigma^{k}=\sum_{j=0}^{N_{*}-1} \sum_{k=0}^{n-1} \psi \circ \sigma^{k N_{*}+j},
$$

\footnotetext{
${ }^{6} 55$ is chosen because $1 / \log 55<1 / 4$, which will be useful in Section 7 .
} 
where $\psi: \Omega \rightarrow \mathbb{R}$, there must exist an integer $0 \leq r_{\omega}<N_{*}$ such that

$$
\lim _{n \rightarrow \infty} \frac{1}{n} \sum_{k=0}^{n-1} \log Q_{\sigma^{-r_{\omega}-k N_{*}(\omega)}}^{\left(N_{*}\right)} \leq \xi .
$$

Now for each $n \in \mathbb{N}$, we may write $n=d_{\omega}+s_{\omega} N_{*}+r_{\omega}$ with $s_{\omega} \geq 0,0 \leq d_{\omega}<N_{*}$. Note that $s_{\omega}$ and $d_{\omega}$ depend on $\omega$ through their dependence on $r_{\omega}$. For the remainder of the proof we will drop the dependence on $\omega$ from our notation and simply refer to $r, s$, and $d$ for $r_{\omega}, s_{\omega}$, and $d_{\omega}$ respectively. Thus, for $f \in \operatorname{BV}\left(X_{\sigma^{-n}(\omega)}\right)$, we can write

$$
\tilde{\mathcal{L}}_{\sigma^{-n}(\omega)}^{n} f=\tilde{\mathcal{L}}_{\sigma^{-r}(\omega)}^{r} \circ \tilde{\mathcal{L}}_{\sigma^{-n+d}(\omega)}^{s N_{*}} \circ \tilde{\mathcal{L}}_{\sigma^{-n}(\omega)}^{d}(f) .
$$

We begin by looking at the outer composition in (4.13). Consider some function $h \in$ $\operatorname{BV}\left(X_{\sigma^{-r}(\omega)}\right)$. Recursively applying Lemma 4.6 and (3.10) gives

$$
\begin{aligned}
\operatorname{var}\left(\tilde{\mathcal{L}}_{\sigma^{-r}(\omega)}^{r}(h)\right) & =\operatorname{var}\left(\tilde{\mathcal{L}}_{\sigma^{-1}(\omega)}\left(\tilde{\mathcal{L}}_{\sigma^{-r}(\omega)}^{r-1} h\right)\right) \\
& \leq L_{\sigma^{-1}(\omega)}\left(\operatorname{var}\left(\tilde{\mathcal{L}}_{\sigma^{-r}(\omega)}^{r-1} h\right)+\nu_{\sigma^{-1}(\omega)}\left(\left|\tilde{\mathcal{L}}_{\sigma^{-r}(\omega)}^{r-1} h\right|\right)\right) \\
& \leq L_{\sigma^{-1}(\omega)}\left(\operatorname{var}\left(\tilde{\mathcal{L}}_{\sigma^{-r}(\omega)}^{r-1} h\right)+\nu_{\sigma^{-r}(\omega)}(|h|)\right) \\
& \leq L_{\sigma^{-1}(\omega)} L_{\sigma^{-2}(\omega)}\left(\operatorname{var}\left(\tilde{\mathcal{L}}_{\sigma^{-r}(\omega)}^{r-2} h\right)+\nu_{\sigma^{-r}(\omega)}(|h|)\right)+L_{\sigma^{-1}(\omega)} \nu_{\sigma^{-r}(\omega)}(h) \\
& \leq \prod_{j=1}^{r} L_{\sigma^{-j}(\omega)} \operatorname{var}(h)+\nu_{\sigma^{-r}(\omega)}(|h|) \cdot \sum_{j=1}^{r} \prod_{k=1}^{j} L_{\sigma^{-k}(\omega)} .
\end{aligned}
$$

As $r<N_{*}$, we can write

$$
\operatorname{var}\left(\tilde{\mathcal{L}}_{\sigma^{-r}(\omega)}^{r}(h)\right) \leq C^{(1)}(\omega)\left(\operatorname{var}(h)+\nu_{\sigma^{-r}(\omega)}(|h|)\right),
$$

where

$$
C^{(1)}(\omega):=N_{*} \cdot \prod_{j=1}^{N_{*}} L_{\sigma^{-j}(\omega)} .
$$

To deal with the innermost composition in (4.13) we will use similar techniques as when dealing with the outer composition. However, we first remark that since $\log L_{\omega} \in L_{m}^{1}(\Omega)$, Birkhoff's Ergodic Theorem implies that

$$
\lim _{k \rightarrow \pm \infty} \frac{1}{|k|} \log L_{\sigma^{k}(\omega)}=0
$$

and thus, we must also have that for each $\delta>0$ there exists some measurable constant $C_{\delta}^{(2)}(\omega) \geq 1$ such that we have

$$
L_{\sigma^{k}(\omega)} \leq C_{\delta}^{(2)}(\omega) e^{\delta|k|}
$$

for each $k \in \mathbb{Z}$. Continuing as in (4.14) together with (4.15), we see

$$
\begin{aligned}
\operatorname{var}\left(\tilde{\mathcal{L}}_{\sigma^{-n}(\omega)}^{d}(f)\right) & \leq \prod_{j=n-d+1}^{n} L_{\sigma^{-j}(\omega)} \operatorname{var}(f)+\sum_{j=n-d+1}^{n} \prod_{k=n-d+1}^{j} L_{\sigma^{-k}(\omega)} \nu_{\sigma^{-n}(\omega)}(|f|) \\
& \leq\left(C_{\delta}^{(2)}(\omega)\right)^{d} \exp \left(\delta \sum_{j=n-d+1}^{n} j\right) \operatorname{var}(f)
\end{aligned}
$$




$$
\begin{aligned}
& +\sum_{j=n-d+1}^{n}\left(C_{\delta}^{(2)}(\omega)\right)^{j-n+d} \exp \left(\delta \sum_{k=n-d+1}^{j} k\right) \nu_{\sigma^{-n}(\omega)}(|f|) \\
\leq & d\left(C_{\delta}^{(2)}(\omega)\right)^{N_{*}} e^{n d \delta}\left(\operatorname{var}(f)+\nu_{\sigma^{-n}(\omega)}(|f|)\right) \\
\leq & C^{(2)}(\omega) e^{n \frac{\varepsilon}{2}}\left(\operatorname{var}(f)+\nu_{\sigma^{-n}(\omega)}(|f|)\right)
\end{aligned}
$$

where $\delta$ has been chosen to be smaller than $\varepsilon / 2 N_{*}$ and

$$
C^{(2)}(\omega):=N_{*}\left(C_{\delta}^{(2)}(\omega)\right)^{N_{*}}
$$

To deal with the middle composition of (4.13) we let $\tau=\sigma^{-n+d}(\omega)$ and consider some function $h \in \mathrm{BV}\left(X_{\tau}\right)$. Applying Corollary 4.4 and (3.10) repeatedly yields

$$
\begin{aligned}
\operatorname{var}\left(\tilde{\mathcal{L}}_{\tau}^{s N_{*}}(h)\right) & =\operatorname{var}\left(\tilde{\mathcal{L}}_{\sigma^{(s-1) N_{*}(\tau)}}^{N_{*}}\left(\tilde{\mathcal{L}}_{\tau}^{(s-1) N_{*}} h\right)\right) \\
& \leq Q_{\sigma^{(s-1) N_{*}(\tau)}}^{\left(N_{*}\right)} \operatorname{var}\left(\tilde{\mathcal{L}}_{\tau}^{(s-1) N_{*}} h\right)+K_{\sigma^{(s-1) N_{*}(\tau)}}^{\left(N_{*}\right)} \nu_{\tau}(|h|) \\
& \leq \prod_{j=1}^{s} Q_{\sigma^{(s-j) N_{*}(\tau)}}^{\left(N_{*}\right)} \operatorname{var}(h)+\sum_{j=1}^{s} \prod_{k=1}^{j}\left(Q_{\sigma^{(s-k) N_{*}(\tau)}}^{\left(N_{*}\right)} K_{\sigma^{(s-j) N_{*}}(\tau)}^{\left(N_{*}\right)}\right) \nu_{\tau}(|h|) .
\end{aligned}
$$

In light of the definitions of $N_{*}$ (4.6) and $\xi$ (4.7) and using (4.12), we can find a measurable constant $C^{(3)}(\omega)>0$ such that for any $j \geq 1$ we have

$$
\prod_{k=1}^{j} Q_{\sigma^{(s-k) N_{*}(\tau)}}^{\left(N_{*}\right)} \leq C^{(3)}(\omega) e^{-\left(\xi-\frac{\varepsilon}{2}\right) j N_{*}} .
$$

Furthermore, since $\log K_{\omega}^{\left(N_{*}\right)} \in L_{m}^{1}(\Omega)$ we can find a measurable constant $C^{(4)}(\omega) \geq C^{(3)}(\omega)$ such that for any $j \geq 1$ we have

$$
K_{\sigma^{(s-j) N_{*}(\tau)}}^{\left(N_{*}\right)} \leq C^{(4)}(\omega) e^{\frac{\varepsilon}{2} j N_{*}} .
$$

Inserting (4.18) and (4.19) into (4.17) gives

$$
\begin{aligned}
\operatorname{var}\left(\tilde{\mathcal{L}}_{\tau}^{s N_{*}}(h)\right) \leq & \prod_{j=1}^{s} Q_{\sigma^{(s-j) N_{*}(\tau)}}^{\left(N_{*}\right)} \operatorname{var}(h)+\sum_{j=1}^{s} \prod_{k=1}^{j} Q_{\sigma^{(s-k) N_{*}}(\tau)}^{\left(N_{*}\right)} K_{\sigma^{(s-j) N_{*}}(\tau)}^{\left(N_{*}\right)} \nu_{\tau}(|h|) \\
\leq & C^{(4)}(\omega) e^{-\left(\xi-\frac{\varepsilon}{2}\right) s N_{*}} \operatorname{var}(h) \\
& \quad+\sum_{j=1}^{s} C^{(4)}(\omega) e^{-\left(\xi-\frac{\varepsilon}{2}\right)(s-j) N_{*}} \cdot C^{(4)}(\omega) e^{\frac{\varepsilon}{2}(s-j) N_{*}} \nu_{\tau}(|h|) \\
\leq & C^{(4)}(\omega) e^{-\left(\xi-\frac{\varepsilon}{2}\right) s N_{*}} \operatorname{var}(h)+\frac{\left(C^{(4)}(\omega)\right)^{2}}{1-e^{-(\xi-\varepsilon) N_{*}}} \nu_{\tau}(|h|) \\
\leq & C^{(5)}(\omega) e^{-\left(\xi-\frac{\varepsilon}{2}\right) s N_{*}} \operatorname{var}(h)+C^{(5)}(\omega) \nu_{\tau}(|h|)
\end{aligned}
$$

where the measurable constant $C^{(5)}(\omega)$ is given by

$$
C^{(5)}(\omega):=\max \left\{C^{(4)}(\omega), \frac{\left(C^{(4)}(\omega)\right)^{2}}{1-e^{-(\xi-\varepsilon) N_{*}}}\right\} .
$$


For the remainder of the proof we will ignore the $\omega$ dependence of the constants $C^{(j)}(\omega)$ and simply write $C^{(j)}$ instead for $j=1, \ldots, 5$. Note that since $s N_{*}=n-d-r$ and since $0 \leq d, r \leq N_{*}$, we can write

$$
-\left(\xi-\frac{\varepsilon}{2}\right) s N_{*}+\frac{n \varepsilon}{2}=-(\xi-\varepsilon) n+\left(\xi-\frac{\varepsilon}{2}\right)(d+r) \leq-(\xi-\varepsilon) n+2\left(\xi-\frac{\varepsilon}{2}\right) N_{*}
$$

Finally, assembling our estimates (4.14), (4.16), and (4.20), in view of (4.21) we get

$$
\begin{aligned}
\operatorname{var} & \left(\tilde{\mathcal{L}}_{\sigma^{-n}(\omega)}^{n} f\right)=\operatorname{var}\left(\tilde{\mathcal{L}}_{\sigma^{-r}(\omega)}^{r} \circ \tilde{\mathcal{L}}_{\sigma^{-n+d}(\omega)}^{s N_{*}} \circ \tilde{\mathcal{L}}_{\sigma^{-n}(\omega)}^{d}(f)\right) \\
& \leq C^{(1)}\left(\operatorname{var}\left(\tilde{\mathcal{L}}_{\sigma^{-n+d}(\omega)}^{s N_{*}} \circ \tilde{\mathcal{L}}_{\sigma^{-n}(\omega)}^{d}(f)\right)+\nu_{\sigma^{-n}(\omega)}(|f|)\right) \\
& \leq C^{(1)}\left(C^{(5)} e^{-\left(\xi-\frac{\varepsilon}{2}\right) s N_{*}} \operatorname{var}\left(\tilde{\mathcal{L}}_{\sigma^{-n}(\omega)}^{d}(f)\right)+\left(C^{(5)}+1\right) \nu_{\sigma^{-n}(\omega)}(|f|)\right) \\
& \leq C^{(1)}\left(C^{(5)} e^{-\left(\xi-\frac{\varepsilon}{2}\right) s N_{*}} C^{(2)} e^{\frac{n \varepsilon}{2}}\left(\operatorname{var}(f)+\nu_{\sigma^{-n}(\omega)}(|f|)\right)+\left(C^{(5)}+1\right) \nu_{\sigma^{-n}(\omega)}(|f|)\right) \\
& \leq C^{(1)} C^{(5)} C^{(2)} e^{2(\xi-\varepsilon) N_{*}} e^{-(\xi-\varepsilon) n}\left(\operatorname{var}(f)+\left(C^{(5)}+1\right) \nu_{\sigma^{-n}(\omega)}(|f|)\right) \\
& \leq C_{\varepsilon}(\omega) e^{-(\xi-\varepsilon) n} \operatorname{var}(f)+C_{\varepsilon}(\omega) \nu_{\sigma^{-n}(\omega)}(|f|),
\end{aligned}
$$

where the measurable constant $C_{\varepsilon}(\omega)>0$ is taken so large that

$$
C_{\varepsilon}(\omega) \geq\left(C^{(1)} C^{(5)} C^{(2)}\right)\left(C^{(5)}+1\right) e^{2(\xi-\varepsilon) N_{*}},
$$

which completes the proof.

\section{Random Birkhoff Cones and Hilbert Metrics}

In this section we first recall the theory of convex cones first used by Birkhoff in [5], and then present the random cones on which our operator $\tilde{\mathcal{L}}_{\omega}$ will act as a contraction. The use of convex cones in dynamical systems was first investigated in [37, 20, 31]. We begin with a definition.

Definition 5.1. Given a vector space $\mathcal{V}$, we call a subset $\mathcal{C} \subseteq \mathcal{V}$ a convex cone if $\mathcal{C}$ satisfies the following:

(1) $\mathcal{C} \cap-\mathcal{C}=\emptyset$

(2) for all $\alpha>0, \alpha \mathrm{e}=\mathrm{C}$,

(3) $\mathrm{C}$ is convex,

(4) for all $f, h \in \mathcal{C}$ and all $\alpha_{n} \in \mathbb{R}$ with $\alpha_{n} \rightarrow \alpha$ as $n \rightarrow \infty$, if $h-\alpha_{n} f \in \mathcal{C}$ for each $n \in \mathbb{N}$, then $h-\alpha f \in \mathcal{C} \cup\{0\}$.

Lemma 5.2 (Lemma 2.1 [33]). The relation $\leq$ defined on $\mathcal{V}$ by

$$
f \leq h \text { if and only if } h-f \in \mathcal{C} \cup\{0\}
$$

is a partial order satisfying the following:

(i) $f \leq 0 \leq f \Longrightarrow f=0$,

(ii) $\lambda>0$ and $f \geq 0 \Longleftrightarrow \lambda f \geq 0$,

(iii) $f \leq h \Longleftrightarrow 0 \leq h-f$,

(iv) for all $\alpha_{n} \in \mathbb{R}$ with $\alpha_{n} \rightarrow \alpha, \alpha_{n} f \leq h \Longrightarrow \alpha f \leq h$,

(v) $f \geq 0$ and $h \geq 0 \Longrightarrow f+h \geq 0$. 
The Hilbert metric on $\mathcal{C}$ is given by the following definition.

Definition 5.3. Define a distance $\Theta(f, h)$ by

$$
\Theta(f, h):=\log \frac{\beta(f, h)}{\alpha(f, h)},
$$

where

$$
\alpha(f, h):=\sup \{a>0: a f \leq h\} \quad \text { and } \quad \beta(f, h):=\inf \{b>0: b f \geq h\} .
$$

Note that $\Theta$ is a pseudo-metric as two elements in the cone may be at an infinite distance from each other. Furthermore, $\Theta$ is a projective metric because any two proportional elements must be zero distance from each other. The next theorem, which is due to Birkhoff [5], shows that every positive linear operator that preserves the cone is a contraction provided that the diameter of the image is finite.

Theorem $5.4([5])$. Let $\mathcal{V}_{1}$ and $\mathcal{V}_{2}$ be vector spaces with convex cones $\mathcal{C}_{1} \subseteq \mathcal{V}_{1}$ and $\mathcal{C}_{2} \subseteq \mathcal{V}_{2}$ and a positive linear operator $\mathcal{L}: \mathcal{V}_{1} \rightarrow \mathcal{V}_{2}$ such that $\mathcal{L}\left(\mathcal{C}_{1}\right) \subseteq \mathcal{C}_{2}$. If $\Theta_{i}$ denotes the Hilbert metric on the cone $\mathcal{C}_{i}$ and if

$$
\Delta=\sup _{f, h \in \mathcal{C}_{1}} \Theta_{2}(\mathcal{L} f, \mathcal{L} h)
$$

then

$$
\Theta_{2}(\mathcal{L} f, \mathcal{L} h) \leq \tanh \left(\frac{\Delta}{4}\right) \Theta_{1}(f, h) .
$$

for all $f, h \in \mathcal{C}_{1}$.

Note that it is not clear whether $(\mathcal{C}, \Theta)$ is complete. The following lemma of [33] addresses this problem by linking the metric $\Theta$ with a suitable norm $\|\cdot\|$ on $\mathcal{V}$.

Lemma 5.5 ([33], Lemma 2.2). Let $\|\cdot\|$ be a norm on $\mathcal{V}$ such that for all $f, h \in \mathcal{V}$ if $-f \leq h \leq f$, then $\|h\| \leq\|f\|$, and let $\varrho: \mathcal{C} \rightarrow(0, \infty)$ be a homogeneous and order-preserving function, which means that for all $f, h \in \mathcal{C}$ with $f \leq h$ and all $\lambda>0$ we have

$$
\varrho(\lambda f)=\lambda \varrho(f) \quad \text { and } \quad \varrho(f) \leq \varrho(h) .
$$

Then, for all $f, h \in \mathcal{\varrho}(f)=\varrho(h)>0$ implies that

$$
\|f-h\| \leq\left(e^{\Theta(f, h)}-1\right) \min \{\|f\|,\|h\|\} .
$$

Remark 5.6. Note that the choice $\varrho(\cdot)=\|\cdot\|$ satisfies the hypothesis, however in the sequel we shall be interested in the choice of $\varrho=\nu_{\omega}$ in Section 8 .

Definition 5.7. For each $a>0$ and $\omega \in \Omega$ let

$$
\mathscr{C}_{\omega, a}:=\left\{f \in \operatorname{BV}\left(X_{\omega}\right): f \geq 0, \operatorname{var}(f) \leq a \nu_{\omega}(f)\right\} .
$$

To see that this cone is non-empty, we note that the function $f+c \in \mathscr{C}_{\omega, a}$ for $f \in \mathrm{BV}$ and $c \geq a^{-1} \operatorname{var}(f)-\inf _{X_{\omega}} f$. We also define the cone

$$
\mathscr{C}_{\omega,+}:=\left\{f \in \operatorname{BV}\left(X_{\omega}\right): f \geq 0\right\} .
$$

Let $\Theta_{\omega, a}$ and $\Theta_{\omega,+}$ denote the Hilbert metrics induced on the respective cones $\mathscr{C}_{\omega, a}$ and $\mathscr{C}_{\omega,+}$. The following lemma collects together the main properties of these metrics. 
Lemma 5.8 ([33], Lemmas 4.2, 4.3, 4.5). For $f, h \in \mathscr{C}_{\omega,+}$ the $\Theta_{\omega,+}$ distance between $f, h$ is given by

$$
\Theta_{\omega,+}(f, h)=\log \sup _{x, y \in X_{\omega}} \frac{f(y) h(x)}{f(x) h(y)}
$$

If $f, h \in \mathscr{C}_{\omega, a}$, then

$$
\Theta_{\omega,+}(f, h) \leq \Theta_{\omega, a}(f, h)
$$

and if $f \in \mathscr{C}_{\omega, a / 2}$ we then have

$$
\Theta_{\omega, a}(\mathbb{1}, f) \leq \log \frac{\|f\|_{\infty}+\frac{1}{2} \nu_{\omega}(f)}{\min \left\{\inf _{X_{\omega}} f, \frac{1}{2} \nu_{\omega}(f)\right\}} .
$$

As a consequence of Lemma 4.1 we have that

$$
\tilde{\mathcal{L}}_{\omega}\left(\mathscr{C}_{\omega,+}\right) \subseteq \mathscr{C}_{\sigma(\omega),+}
$$

and thus $\tilde{\mathcal{L}}_{\omega}$ is a weak contraction on $\mathscr{C}_{\omega,+}$. Our eventual goal is to show a similar statement for the cones $\mathscr{C}_{\omega, a}$. We begin that journey with the following lemma, a version of which first appeared in [32, Lemma 3.2].

Lemma 5.9. Given $a>0$, for each $\omega \in \Omega$ there exists a finite partition $\mathcal{U}_{\omega, a}$ of $X_{\omega}$ and a positive integer $t_{\omega, a}$ such that

$$
b_{\omega}:=\sup _{U \in \mathcal{U}_{\omega, a}}\left\|\frac{\mathcal{L}_{\omega}^{t_{\omega, a}} \mathbb{1}_{U}}{\mathcal{L}_{\omega}^{t_{\omega, a}} \mathbb{1}_{\omega}}\right\|_{\infty}<\frac{1}{2 a}
$$

and for all $f \in \mathscr{C}_{\omega, a}$ there exists $U_{f} \in \mathcal{U}_{\omega, a}$ such that inf $\left.f\right|_{U_{f}} \geq \frac{1}{2} \nu_{\omega}(f)$.

Proof. First, we let $t_{\omega, a} \in \mathbb{N}$ be given by

$$
t_{\omega, a}:=\min \left\{n_{0} \in \mathbb{N}: \frac{\left\|g_{\omega}^{(n)}\right\|_{\infty}}{\inf \mathcal{L}_{\omega}^{n} \mathbb{1}_{\omega}}<\frac{1}{2 a} \text { for all } n \geq n_{0}\right\} .
$$

Then for each $U \in z_{\omega}^{\left(t_{\omega, a}\right)}$ we have

$$
\left\|\frac{\mathcal{L}_{\omega}^{t_{\omega, a}} \mathbb{1}_{U}}{\mathcal{L}_{\omega}^{t_{\omega, a}} \mathbb{1}_{\omega}}\right\|_{\infty} \leq \frac{\left\|g_{\omega}^{\left(t_{\omega, a}\right)}\right\|_{\infty}}{\inf \mathcal{L}_{\omega}^{t_{\omega, a}} \mathbb{1}_{\omega}}<\frac{1}{2 a}
$$

If $\mathcal{Z}_{\omega}^{\left(t_{\omega, a}\right)}$ is finite then we may set $\mathcal{U}_{\omega, a}:=z_{\omega}^{\left(t_{\omega, a}\right)}$. If, on the other hand, $\mathcal{Z}_{\omega}^{\left(t_{\omega, a}\right)}$ is infinite, noting that Lemma 2.4 gives

$$
\mathcal{L}_{\omega}^{t_{\omega, a}} \mathbb{1}_{\omega}=\sum_{U \in \mathcal{Z}_{\omega}^{\left(t_{\omega}, a\right.}} \mathcal{L}_{\omega}^{t_{\omega, a}} \mathbb{1}_{U} \leq \sum_{U \in \mathcal{Z}_{\omega}^{\left(t_{\omega}, a\right)}}\left\|\mathcal{L}_{\omega}^{t_{\omega, a}} \mathbb{1}_{U}\right\|_{\infty} \leq S_{\omega}^{\left(t_{\omega, a}\right)}<\infty,
$$

we can choose intervals $U_{1}, \ldots, U_{\ell} \in Z_{\omega}^{\left(t_{\omega, a}\right)}$ such that

$$
\left\|\frac{\mathcal{L}_{\omega}^{t_{\omega, a}} \mathbb{1}_{X_{\omega} \backslash \cup_{j=1}^{\ell} U_{j}}}{\mathcal{L}_{\omega}^{t_{\omega, a}} \mathbb{1}_{\omega}}\right\|_{\infty}<\frac{1}{2 a} .
$$


Now the set $X_{\omega} \backslash \cup_{j=1}^{\ell} U_{j}$ must consist of at most $\ell+1$ many intervals (which are not necessarily elements of $Z_{\omega}^{\left(t_{\omega, a}\right)}$, though they are unions of elements of $\left.Z_{\omega}^{\left(t_{\omega, a}\right)}\right)$, say $U_{\ell+1}, \ldots, U_{L}$. Setting $\mathcal{U}_{\omega, a}=\left\{U_{j}: 1 \leq j \leq L\right\}$ finishes the first claim. Now fix $f \in \mathscr{C}_{\omega, a}$ and set

$$
\mathcal{A}=\left\{U \in \mathcal{U}_{\omega, a}: \text { there exists } x_{U} \in U \text { such that } f\left(x_{U}\right)<\frac{1}{2} \nu_{\omega}(f)\right\} .
$$

To prove the second part of Lemma 5.9 we will show that $\mathcal{A} \neq \mathcal{U}_{\omega, a}$. Towards contradiction, suppose that for each $U \in \mathcal{U}_{\omega, a}$ there exists $x_{U} \in U$ such that $f\left(x_{U}\right)<1 / 2 \nu_{\omega}(f)$. Now, for $n \geq t_{\omega, a}$ we have

$$
\mathcal{L}_{\omega}^{n}\left(f \mathbb{1}_{U}\right) \leq \mathcal{L}_{\omega}^{n}\left(\mathbb{1}_{U}\right)\left(f\left(x_{U}\right)+\operatorname{var}_{U}(f)\right)<\mathcal{L}_{\omega}^{n}\left(\mathbb{1}_{U}\right) \frac{\nu_{\omega}(f)}{2}+b_{\omega} \mathcal{L}_{\omega}^{n}\left(\mathbb{1}_{\omega}\right) \operatorname{var}_{U}(f) .
$$

Summing over all $U \in \mathcal{U}_{\omega, a}$ gives

$$
\mathcal{L}_{\omega}^{n} f<\mathcal{L}_{\omega}^{n_{\omega}} \mathbb{1}_{\omega} \cdot \frac{\nu_{\omega}(f)}{2}+b_{\omega} \mathcal{L}_{\omega}^{n_{\omega}} \mathbb{1}_{\omega} \cdot \operatorname{var}(f) .
$$

Now, integrating both sides with respect to $\nu_{\sigma^{n}(\omega)}$ and then dividing by $\lambda_{\omega}^{n}$ gives

$$
\frac{\nu_{\sigma^{n}(\omega)}\left(\mathcal{L}_{\omega}^{n} f\right)}{\lambda_{\omega}^{n}}<\frac{\nu_{\omega}(f)}{2}+b_{\omega} \operatorname{var}(f) \leq\left(\frac{1}{2}+a b_{\omega}\right) \nu_{\omega}(f)
$$

As the left hand side is equal to $\nu_{\omega}(f)$ and noting that $a b_{\omega}<1 / 2$, we have arrived at our desired contradiction, and thus, we are done.

As an immediate consequence of the previous lemma and its proof we may define the measurable function $N_{\omega, a}\left(\mathcal{U}_{\omega, a}\right): \Omega \rightarrow \mathbb{N}$ by

$$
N_{\omega, a}\left(\mathcal{U}_{\omega, a}\right):=\max \left\{M_{\omega}(U): U \in \mathcal{U}_{\omega, a}\right\} \geq t_{\omega, a} .
$$

\section{Cone Contraction on Good Fibers}

In this section we follow Buzzi's approach [10], and describe the good behavior across a large measure set of fibers. In particular, we will show that, for sufficiently many iterates $R_{*}$, the normalized transfer operator $\tilde{\mathcal{L}}_{\omega}^{R_{*}}$ uniformly contracts the cone $\mathscr{C}_{\omega, a}$ on "good" fibers $\omega$. However, first we must describe what exactly makes a fiber "good".

Recall that the numbers $\xi$ and $\rho$ are given by

$$
\xi:=-\frac{1}{N_{*}} \int_{\Omega} \log Q_{\omega}^{\left(N_{*}\right)} d m(\omega) \quad \text { and } \quad \rho:=\frac{1}{N_{*}} \int_{\Omega} \log L_{\omega}^{N_{*}} d m(\omega) .
$$

Note that (4.9), (4.10), and the ergodic theorem imply that

$$
\log 55 \leq \rho=\lim _{n \rightarrow \infty} \frac{1}{n N_{*}} \sum_{k=0}^{n-1} \log L_{\sigma^{k N *}(\omega)}^{N_{*}} .
$$

The following definition is adapted from [10, Definition 2.4].

Definition 6.1. We will say that $\omega$ is $\operatorname{good}$ with respect to the numbers $\varepsilon, a, B_{*}, R_{a}=$ $q_{a} N_{*}, C_{*} \geq 1$, and $0<\alpha_{*} \leq C_{*}$ if the following:

$$
B_{*} q_{a} e^{-\frac{\xi}{2} R_{a}} \leq \frac{1}{3}
$$


(G2) $\frac{1}{R_{a}} \sum_{k=0}^{R_{a} / N_{*}-1} \log L_{\sigma^{k N *}(\omega)}^{N_{*}} \in[\rho-\varepsilon, \rho+\varepsilon]$,

(G3) $R_{a} \geq N_{\omega, a}\left(\mathcal{U}_{\omega, a}\right)$,

(G4) $C_{*}^{-1} \leq \inf \mathcal{L}_{\omega}^{R_{a}} \mathbb{1}_{\omega} \leq \lambda_{\omega}^{R_{a}} \leq\left\|\mathcal{L}_{\omega}^{R_{a}} \mathbb{1}_{\omega}\right\|_{\infty} \leq C_{*}$,

(G5) inf $\mathcal{L}_{\omega}^{R_{a}} \mathbb{1}_{U} \geq \alpha_{*}$ for all $U \in \mathcal{U}_{\omega, a}$.

Now, we denote

$$
\varepsilon_{0}:=\min \left\{1, \frac{\xi}{2}\right\} .
$$

The following lemma describes the prevalence of the good fibers as well as how to find them.

Lemma 6.2. Given $\varepsilon<\varepsilon_{0}$ and $a>0$, there exists parameters $B_{*}, R_{a}, \alpha_{*}, C_{*}$ (all of which depend on $\varepsilon)$ such that there is a set $\Omega_{G} \subseteq \Omega$ of good $\omega$ with $m\left(\Omega_{G}\right) \geq 1-\varepsilon / 4$.

Proof. We begin by letting

$$
\Omega^{\prime}=\Omega^{\prime}\left(B_{*}\right):=\left\{\omega \in \Omega: C_{\varepsilon}(\omega) \leq B_{*}\right\},
$$

where $C_{\varepsilon}(\omega)>0$ is the $m$-a.e. finite measurable constant coming from Proposition 4.9. Choose $B_{*}$ sufficiently large such that $m\left(\Omega^{\prime}\right) \geq 1-\varepsilon / 8$. Then for any $\omega \in \Omega$ with $\sigma^{q N_{*}}(\omega) \in \Omega^{\prime}$ for any $q \in \mathbb{N}$, Proposition 4.9 gives

$$
\operatorname{var}\left(\tilde{\mathcal{L}}_{\omega}^{q N_{*}} f\right) \leq B_{*} e^{-(\xi-\varepsilon) q N_{*}} \operatorname{var}(f)+B_{*} \nu_{\omega}(f)
$$

for any $f \in \operatorname{BV}\left(X_{\omega}\right)$. Noting that $\varepsilon<\xi / 2$ by (6.2), we set $R_{0}=q_{0} N_{*}$ and choose $q_{0}$ sufficiently large such that

$$
B_{*} q_{0} e^{-(\xi-\varepsilon) R_{0}} \leq B_{*} q_{0} e^{-\frac{\xi}{2} R_{0}} \leq \frac{1}{3} .
$$

Now for $q_{1} \geq q_{0}$ and define the set

$$
\Omega^{\prime \prime}=\Omega^{\prime \prime}\left(q_{1}, C_{*}, \alpha_{*}\right):=\left\{\omega \in \Omega:(\mathrm{G} 2)-(\mathrm{G} 5) \text { hold for the value } R_{1}=q_{1} N_{*}\right\} .
$$

Now choose $q_{a} \geq q_{1}, C_{*}$, and $\alpha_{*}$, with $C_{*} \geq \alpha_{*}$, such that $m\left(\Omega^{\prime \prime}\left(q_{a}, C_{*}, \alpha_{*}\right)\right) \geq 1-\varepsilon / 8$. Set $R_{a}:=q_{a} N_{*}$. Set $\Omega_{G}:=\Omega^{\prime \prime} \cap \sigma^{-R_{a}}\left(\Omega^{\prime}\right)$. Then $\Omega_{G}$ is the set of all $\omega \in \Omega$ which are good with respect to the numbers $B_{*}, R_{a}, \alpha_{*}, C_{*}$ and $m\left(\Omega_{G}\right) \geq 1-\varepsilon / 4$, and so we are done.

In what follows, given a value $B_{*}$, we will consider cone parameters

$$
a \geq a_{0}:=6 B_{*}
$$

and we set

$$
q_{*}=q_{a_{0}} \quad \text { and } \quad R_{*}:=R_{a_{0}}=q_{*} N_{*} .
$$

Note that (G1) together with Proposition 4.9 implies that, for $\varepsilon<\varepsilon_{0}$ and $\omega \in \Omega_{G}$, we have

$$
\begin{aligned}
\operatorname{var}\left(\tilde{\mathcal{L}}_{\omega}^{R_{*}} f\right) & \leq B_{*} e^{-(\xi-\varepsilon) R_{*}} \operatorname{var}(f)+B_{*} \nu_{\omega}(f) \\
& \leq B_{*} q_{*} e^{-\frac{\xi}{2} R_{*}} \operatorname{var}(f)+B_{*} \nu_{\omega}(f) \\
& \leq \frac{1}{3} \operatorname{var}(f)+B_{*} \nu_{\omega}(f) .
\end{aligned}
$$


For each $\omega \in \Omega, a>0$, and some set $Y \subseteq \mathscr{C}_{\omega, a}$ we let

$$
\operatorname{diam}_{\omega, a}(Y):=\sup _{x, y \in Y} \Theta_{\omega, a}(x, y)
$$

and

$$
\operatorname{diam}_{\omega,+}(Y):=\sup _{x, y \in Y} \Theta_{\omega,+}(x, y)
$$

denote the diameter of $Y$ in the respective cones $\mathscr{C}_{\omega, a}$ and $\mathscr{C}_{\omega,+}$ with respect to the respective metrics $\Theta_{\omega, a}$ and $\Theta_{\omega,+}$. We come now to the final, and main, result of this section which shows that the normalized operator is a contraction on the fiber cones $\mathscr{C}_{\omega, a}$ and that the image has finite diameter.

Lemma 6.3. If $\omega$ is good with respect to the numbers $\varepsilon, a_{0}, B_{*}, R_{*}, \alpha_{*}, C_{*}$ then for each $a \geq a_{0}$ we have

$$
\tilde{\mathcal{L}}_{\omega}^{R_{*}}\left(\mathscr{C}_{\omega, a}\right) \subseteq \mathscr{C}_{\sigma_{*}(\omega), a / 2} \subseteq \mathscr{C}_{\sigma_{*}(\omega), a}
$$

and that

$$
\operatorname{diam}_{\sigma_{*}(\omega), a}\left(\tilde{\mathcal{L}}_{\omega}^{R_{*}}\left(\mathscr{C}_{\omega, a}\right)\right) \leq \Delta_{a}:=2 \log \frac{C_{*}(3+a)}{\alpha_{*}}<\infty .
$$

Proof. For $\omega$ good and $f \in \mathscr{C}_{\omega, a},(6.7),(6.5)$, and (3.10) give

$$
\begin{aligned}
\operatorname{var}\left(\tilde{\mathcal{L}}_{\omega}^{R_{*}} f\right) & \leq \frac{1}{3} \operatorname{var}(f)+B_{*} \nu_{\omega}(f) \leq\left(\frac{a}{3}+B_{*}\right) \nu_{\omega}(f) \\
& \leq\left(\frac{a}{3}+\frac{a}{6}\right) \nu_{\omega}(f)=\frac{a}{2} \nu_{\sigma^{R *}(\omega)}\left(\tilde{\mathcal{L}}_{\omega}^{R_{*}} f\right) .
\end{aligned}
$$

Hence we have

$$
\tilde{\mathcal{L}}_{\omega}^{R_{*}}\left(\mathscr{C}_{\omega, a}\right) \subseteq \mathscr{C}_{\sigma^{R *}(\omega), a / 2} \subseteq \mathscr{C}_{\sigma^{R *}(\omega), a}
$$

as desired. Now, towards finding the diameter of $\tilde{\mathcal{L}}_{\omega}^{R_{*}}\left(\mathscr{C}_{\omega, a}\right)$ in $\mathscr{C}_{\sigma^{R}(\omega), a}$ we note that Lemma 5.8 implies

$$
\Theta_{\sigma^{R_{*}}(\omega), a}\left(\tilde{\mathcal{L}}_{\omega}^{R_{*}} f, \mathbb{1}_{\sigma^{R_{*}(\omega)}}\right) \leq \log \frac{\left\|\tilde{\mathcal{L}}_{\omega}^{R_{*}} f\right\|_{\infty}+\frac{1}{2} \nu_{\sigma^{R_{*}(\omega)}}\left(\tilde{\mathcal{L}}_{\omega}^{R_{*}} f\right)}{\min \left\{\inf \tilde{\mathcal{L}}_{\omega}^{R_{*}} f, \frac{1}{2} \nu_{\sigma^{R_{*}(\omega)}}\left(\tilde{\mathcal{L}}_{\omega}^{R_{*}} f\right)\right\}} .
$$

Thus, to finish the proof we look for bounds for $\left\|\tilde{\mathcal{L}}_{\omega}^{R_{*}} f\right\|_{\infty}$ and inf $\tilde{\mathcal{L}}_{\omega}^{R_{*}} f$ in terms of $\nu_{\sigma^{*}(\omega)}\left(\tilde{\mathcal{L}}_{\omega}^{R_{*}} f\right)$. To that end, we note that since $\tilde{\mathcal{L}}_{\omega}^{R_{*}} f \in \mathscr{C}_{\sigma} R_{*}(\omega), a / 2$ we have

$$
\left\|\tilde{\mathcal{L}}_{\omega}^{R_{*}} f\right\|_{\infty} \leq \nu_{\sigma_{*} R^{(\omega)}}\left(\tilde{\mathcal{L}}_{\omega}^{R_{*}} f\right)+\operatorname{var}\left(\tilde{\mathcal{L}}_{\omega}^{R_{*}} f\right) \leq\left(1+\frac{a}{2}\right) \nu_{\sigma *(\omega)}\left(\tilde{\mathcal{L}}_{\omega}^{R_{*}} f\right) .
$$

Since $R_{*} \geq N_{\omega, a_{0}}\left(\mathcal{U}_{\omega, a_{0}}\right) \geq t_{\omega, a_{0}}$ we may apply Lemma 5.9 to find $U_{f} \in \mathcal{U}_{\omega, a_{0}}$, which, taken together with $(\mathrm{G} 4)-(\mathrm{G} 5)$, gives

$$
\inf \tilde{\mathcal{L}}_{\omega}^{R_{*}} f \geq \inf \tilde{\mathcal{L}}_{\omega}^{R_{*}}\left(f \mathbb{1}_{U_{f}}\right) \geq \frac{1}{2} \nu_{\omega}(f) \inf \tilde{\mathcal{L}}_{\omega}^{R_{*}} \mathbb{1}_{U_{f}} \geq \frac{1}{2} \nu_{\omega}(f) \frac{\alpha_{*}}{\lambda_{\omega}^{R_{*}}} \geq \frac{\alpha_{*}}{2 C_{*}} \nu_{\sigma^{R_{*}}(\omega)}\left(\tilde{\mathcal{L}}_{\omega}^{R_{*}} f\right)
$$

Inserting (6.9) and (6.10) into (6.8), simplifying, and applying the triangle inequality finishes the proof. 


\section{Dealing With Bad Fibers}

In this section we again follow Buzzi [10] to show that for fibers in the small measure set of "bad" fibers, $\Omega_{B}:=\Omega \backslash \Omega_{G}$, the cone $\mathscr{C}_{\omega, a}$ of positive functions is invariant after sufficiently many iterations for sufficiently large parameters $a>0$. We accomplish this by introducing the concept of coating intervals, which we then show make up a relatively small portion of an orbit.

Recall that $R_{*}$ is given by (6.6), and for each $\omega \in \Omega$ we let

$$
0 \leq y_{*}(\omega)<R_{*}
$$

be the smallest integer such that for either choice of sign + or - we have

$$
\begin{aligned}
& \lim _{m \rightarrow \infty} \frac{1}{m} \#\left\{0 \leq k<m: \sigma^{ \pm k R_{*}+y_{*}(\omega)}(\omega) \in \Omega_{G}\right\}>1-\varepsilon \\
& \lim _{m \rightarrow \infty} \frac{1}{m} \#\left\{0 \leq k<m: C_{\varepsilon}\left(\sigma^{ \pm k R_{*}+y_{*}(\omega)}(\omega)\right) \leq B_{*}\right\}>1-\varepsilon .
\end{aligned}
$$

This is possible, since by e.g. Lemma 33 [22] ergodicity of $\sigma$ yields the existence of a $\sigma^{R_{*}}$ invariant subset $E \subset \Omega$ and a divisor $d$ of $R_{*}$ such that the ergodic components of $m$ under $\sigma^{R_{*}}$ are the sets $\sigma^{-i}(E), i=0, \ldots, d-1$, and $m(E)=1 / d$. Thus, for $m$-a.e. $\omega \in \Omega$, both limits (7.2) and (7.3) exist by Birkhoff. When $\sigma^{y *(\omega)}(\omega) \in \sigma^{-i}(E)$, by Birkhoff the limit (7.2) equals $m\left(\sigma^{-i}(E) \cap \Omega_{G}\right) \cdot d$. Similarly, since $\sigma^{-R_{*}}\left(\Omega^{\prime}\right) \subset \Omega_{G}$ (proof of Lemma 6.2), and (7.2) is a stronger condition by the definition of $\Omega_{G}$, the limit $(7.3)$ is not less than $m\left(\sigma^{-i}(E) \cap \Omega_{G}\right) \cdot d$. Since $m\left(\Omega_{G}\right)>1-\varepsilon / 4$ and since $\Omega_{G}$ is fixed, we may choose $y_{*}(\omega)$ so that the limits (7.2) and (7.3) are not less than $1-(\varepsilon / 4) \cdot d$ (this worst case is achieved when the mass of $\Omega_{G}$ is distributed equally amongst the $\left.\sigma^{-i}(E), i=0, \ldots, d-1\right)$.

Clearly, $y_{*}: \Omega \rightarrow \mathbb{N}$ is a measurable function such that

$$
\begin{aligned}
& y_{*}\left(\sigma^{y_{*}(\omega)}(\omega)\right)=0 \\
& y_{*}\left(\sigma^{R_{*}}(\omega)\right)=y_{*}(\omega)
\end{aligned}
$$

In particular, (7.4) and (7.5) together imply that

$$
y_{*}\left(\sigma^{y_{*}(\omega)+k R_{*}}(\omega)\right)=0
$$

for all $k \in \mathbb{N}$. Let

$$
\Gamma(\omega):=\prod_{k=0}^{q_{*}-1} L_{\sigma^{k N_{*}(\omega)}}^{N_{*}}
$$

and for each $\omega \in \Omega$ we define the coating length $\ell(\omega)$ as follows:

- if $\omega \in \Omega_{G}$ then set $\ell(\omega):=1$,

- if $\omega \in \Omega_{B}$ then

$$
\ell(\omega):=\min \left\{n \in \mathbb{N}: \frac{1}{n} \sum_{0 \leq k<n}\left(\mathbb{1}_{\Omega_{B}} \log \Gamma\right)\left(\sigma^{k R_{*}}(\omega)\right) \leq \log \varepsilon^{\frac{1}{2}} \rho R_{*}\right\} .
$$

If the minimum is not attained we set $\ell(\omega)=\infty$.

Since $L_{\omega}^{N_{*}} \geq 55^{N_{*}}$ by Lemma 4.6, we must have that

$$
\Gamma(\omega) \geq 55^{R_{*}}
$$


for all $\omega \in \Omega$. It follows from Lemma 4.6 that for $m$-a.e. $\omega \in \Omega$ we have

$$
\operatorname{var}\left(\tilde{\mathcal{L}}_{\omega}^{R_{*}} f\right) \leq L_{\omega}^{R_{*}}\left(\operatorname{var}(f)+\nu_{\omega}(f)\right)=\Gamma(\omega)\left(\operatorname{var}(f)+\nu_{\omega}(f)\right) .
$$

Furthermore, if $\omega \in \Omega_{G}$ it follows from (G2) that

$$
R_{*}(\rho-\varepsilon) \leq \log \Gamma(\omega) \leq R_{*}(\rho+\varepsilon) .
$$

Now let

$$
\varepsilon<\varepsilon_{1}:=\min \left\{\left(\frac{\rho}{2+\rho}\right)^{2},\left(\frac{\log 55}{\rho}\right)^{2}\right\} .
$$

The following proposition collects together some of the key properties of the coating length $\ell(\omega)$.

Proposition 7.1. Concerning the number $\ell(\omega)$, we have the following.

(i) For $m$-a.e. $\omega \in \Omega$ such that $y_{*}(\omega)=0$ we have $\ell(\omega)<\infty$,

(ii) If $\omega \in \Omega_{B}$ then $\ell(\omega) \geq 2$.

Proof. To see (i) we first note that

$$
\frac{1}{n} \sum_{0<k \leq n}\left(\mathbb{1}_{\Omega_{B}} \log \Gamma\right)\left(\sigma^{k R_{*}}(\omega)\right)=\frac{1}{n} \sum_{0<k \leq n} \log \Gamma\left(\sigma^{k R_{*}}(\omega)\right)-\frac{1}{n} \sum_{0<k \leq n}\left(\mathbb{1}_{\Omega_{G}} \log \Gamma\right)\left(\sigma^{k R_{*}}(\omega)\right) .
$$

Towards estimating each of the terms in the right-hand side of $(7.13)$, we recall that $R_{*}=$ $q_{a_{0}} N_{*}$ and use (7.7) and (6.1) to see that

$$
\frac{1}{n} \sum_{0<k \leq n} \log \Gamma\left(\sigma^{k R_{*}}(\omega)\right)=\frac{1}{n} \sum_{k=0}^{n-1} \sum_{j=0}^{q_{a_{0}}-1} \log L_{\sigma^{j N_{*}+k R_{*}}(\omega)}^{N_{*}} \leq(\rho+\varepsilon) R_{*}
$$

for all $n \in \mathbb{N}$ sufficiently large, while (7.2) (with $y_{*}(\omega)=0$ ) together with (7.11) ensure that

$$
\frac{1}{n} \sum_{0<k \leq n}\left(\mathbb{1}_{\Omega_{G}} \log \Gamma\right)\left(\sigma^{k R_{*}}(\omega)\right) \geq(1-\varepsilon)\left((\rho-\varepsilon) R_{*}\right)
$$

for all $n \in \mathbb{N}$ sufficiently large. Now, combining (7.14) and (7.15) together with (7.13) and then using (7.12), for $n \in \mathbb{N}$ sufficiently large we have

$$
\begin{aligned}
\frac{1}{n} \sum_{0<k \leq n}\left(\mathbb{1}_{\Omega_{B}} \log \Gamma\right)\left(\sigma^{k R_{*}}(\omega)\right) & \leq(\rho+\varepsilon) R_{*}-(1-\varepsilon)\left((\rho-\varepsilon) R_{*}\right) \\
& \leq \varepsilon(2+\rho) R_{*} \\
& \leq \varepsilon^{\frac{1}{2}} \rho R_{*} .
\end{aligned}
$$

In light of the definition of $\ell(\omega),(7.8)$, we see that $\ell(\omega)<\infty$.

Now to see (ii) we suppose that $\omega \in \Omega_{B}$ and derive a contradiction if $\ell(\omega)=1$. Indeed, in light of $(7.9)$, we see that $\ell(\omega)=1$ implies that

$$
R_{*} \log 55 \leq \log \Gamma(\omega) \leq \varepsilon^{\frac{1}{2}} \rho R_{*},
$$

and thus that

$$
\varepsilon \geq\left(\frac{\log 55}{\rho}\right)^{2}
$$


which contradicts the fact that we have chosen $\varepsilon<\varepsilon_{1}$.

Remark 7.2. Given $\omega_{0} \in \Omega$, for each $j \geq 0$ let $\omega_{j+1}=\sigma^{\ell\left(\omega_{j}\right) R_{*}}\left(\omega_{j}\right)$. As a consequence of Proposition 7.1 item (i) and (7.5), we see that for $m$-a.e. $\omega_{0} \in \Omega$ with $y_{*}\left(\omega_{0}\right)=0$, we must have that $\ell\left(\omega_{j}\right)<\infty$ for all $j \geq 0$.

Definition 7.3. We will call a (finite) sequence $\omega, \sigma(\omega), \ldots, \sigma^{\ell(\omega) R_{*}-1}(\omega)$ of $\ell(\omega) R_{*}$ many fibers a good block (originating at $\omega$ ) if $\omega \in \Omega_{G}$ (which implies that $\ell(\omega)=1$ ). If, on the other hand, $\omega \in \Omega_{B}$ we call such a sequence a bad block (originating at $\omega$ ).

For each $\omega \in \Omega$ we define the coating intervals along the orbit starting at $\omega$ to be bad blocks of the form $\sigma^{a_{j} R_{*}}(\omega), \sigma^{a_{j} R_{*}+1}(\omega), \ldots, \sigma^{b_{j} R_{*}-1}(\omega)$ where $a_{j}, b_{j}$ are given by

$$
a_{j}:=\min \left\{k \geq b_{j-1}: \sigma^{k R_{*}}(\omega) \in \Omega_{B}\right\} \quad \text { and } \quad b_{j}=a_{j}+\ell\left(\sigma^{a_{j} R_{*}}(\omega)\right)
$$

for $j \geq 1$ and setting $b_{0}:=-1$.

Choosing $\varepsilon<\varepsilon_{1}$, we may define the number

$$
\gamma=\gamma(\varepsilon):=\frac{\varepsilon^{\frac{1}{2}} \rho R_{*}}{R_{*} \log 55}<1 .
$$

Note that since $1 / \log 55<1 / 4$ we can find $\varepsilon_{2}<\varepsilon_{1}$ sufficiently small such that $\gamma<1 / 4$. Now we set

$$
\varepsilon_{3}:=\min \left\{\varepsilon_{2},\left(\frac{\xi}{8 \rho}\right)^{2}\right\} .
$$

Observation 7.4. By our choice of $\varepsilon<\varepsilon_{3}$ we must have that

$$
-\frac{\xi}{2}>2 \varepsilon^{\frac{1}{2}} \rho-(\xi-\varepsilon)(1-\gamma)>\varepsilon^{\frac{1}{2}} \rho-(\xi-\varepsilon)(1-\gamma) .
$$

To see this we note that $\varepsilon<\varepsilon_{3}$ implies that

$$
2 \varepsilon^{\frac{1}{2}} \rho<\frac{\xi}{4} \quad \text { and } \quad \gamma<\frac{1}{4}
$$

which in turn implies that

$$
2 \varepsilon^{\frac{1}{2}} \rho-(\xi-\varepsilon)(1-\gamma)<\frac{\xi}{4}+\xi(\gamma-1)<\frac{\xi}{4}-\frac{3 \xi}{4}=-\frac{\xi}{2}
$$

as desired.

The next lemma shows that the normalized operator is weakly contracting (i.e. nonexpanding) on the fiber cones $\mathscr{C}_{\omega, a}$ for sufficiently large values of $a>a_{0}$.

Lemma 7.5. For every $\varepsilon<\varepsilon_{3}$ and all $\omega \in \Omega$ with $\ell(\omega)<\infty$ we have that

$$
\tilde{\mathcal{L}}_{\omega}^{\ell(\omega) R_{*}}\left(\mathscr{C}_{\omega, a_{*}}\right) \subseteq \mathscr{C}_{\sigma^{\ell(\omega) R_{*}}(\omega), a_{*}}
$$

where

$$
a_{*}=a_{*}(\varepsilon):=a_{0} e^{\varepsilon^{\frac{1}{2}} \rho R_{*}}=6 B_{*} e^{\varepsilon^{\frac{1}{2}} \rho R_{*}} .
$$


Proof. Lemma 6.3 covers the case of $\omega \in \Omega_{G}$, so suppose $\omega \in \Omega_{B}$. Throughout the proof we will denote $\ell(\omega)=\ell \geq 2$. Using (6.4) on good fibers and (7.10) on bad fibers, for any $p \geq 1$ and $f \in \mathscr{C}_{\omega,+}$ we have

$$
\operatorname{var}\left(\tilde{\mathcal{L}}_{\omega}^{p R_{*}} f\right) \leq\left(\prod_{j=0}^{p-1} \Phi_{\sigma^{j R_{*}(\omega)}}^{\left(R_{*}\right)}\right) \operatorname{var}(f)+\sum_{j=0}^{p-1}\left(D_{\sigma^{j R *}(\omega)}^{\left(R_{*}\right)} \cdot \prod_{k=j+1}^{p-1} \Phi_{\sigma^{k R_{*}(\omega)}}^{\left(R_{*}\right)}\right) \nu_{\omega}(f),
$$

where

$$
\Phi_{\tau}^{\left(R_{*}\right)}= \begin{cases}B_{*} e^{-(\xi-\varepsilon) R_{*}} & \text { for } \tau \in \Omega_{G} \\ \Gamma(\tau) & \text { for } \tau \in \Omega_{B}\end{cases}
$$

and

$$
D_{\tau}^{\left(R_{*}\right)}= \begin{cases}B_{*} & \text { for } \tau \in \Omega_{G} \\ \Gamma(\tau) & \text { for } \tau \in \Omega_{B}\end{cases}
$$

For any $0 \leq j<\ell$ we can write

$$
\sum_{0 \leq k<\ell}\left(\mathbb{1}_{\Omega_{B}} \log \Gamma\right)\left(\sigma^{k R_{*}}(\omega)\right)=\sum_{0 \leq k<j}\left(\mathbb{1}_{\Omega_{B}} \log \Gamma\right)\left(\sigma^{k R_{*}}(\omega)\right)+\sum_{j \leq k<\ell}\left(\mathbb{1}_{\Omega_{B}} \log \Gamma\right)\left(\sigma^{k R_{*}}(\omega)\right) .
$$

The definition of $\ell(\omega),(7.8)$, then implies that

$$
\frac{1}{j} \sum_{0 \leq k<j}\left(\mathbb{1}_{\Omega_{B}} \log \Gamma\right)\left(\sigma^{k R_{*}}(\omega)\right)>\varepsilon^{\frac{1}{2}} \rho R_{*}
$$

and consequently that

$$
\frac{1}{\ell-j} \sum_{j \leq k<\ell}\left(\mathbb{1}_{\Omega_{B}} \log \Gamma\right)\left(\sigma^{k R_{*}}(\omega)\right) \leq \varepsilon^{\frac{1}{2}} \rho R_{*} .
$$

Now, using (7.9), (7.24), and (7.18) we see that the proportion of bad blocks is given by

$$
\begin{aligned}
\frac{1}{\ell-j} \#\left\{j \leq k<\ell: \sigma^{k R_{*}}(\omega) \in \Omega_{B}\right\} & =\frac{1}{\ell-j} \sum_{j \leq k<\ell}\left(\mathbb{1}_{\Omega_{B}}\right)\left(\sigma^{k R_{*}}(\omega)\right) \\
& \leq \frac{1}{(\ell-j) R_{*} \log 55} \sum_{j \leq k<\ell}\left(\mathbb{1}_{\Omega_{B}} \log \Gamma\right)\left(\sigma^{k R_{*}}(\omega)\right) \leq \gamma .
\end{aligned}
$$

In view of (7.22), using (7.24), (7.25), for any $0 \leq j<\ell$ we have

$$
\begin{aligned}
\prod_{k=j}^{\ell-1} \Phi_{\sigma^{k R_{*}(\omega)}}^{\left(R_{*}\right)} & =\prod_{\substack{j \leq k<\ell \\
\sigma^{k R_{*}}(\omega) \in \Omega_{G}}} B_{*} e^{-(\xi-\varepsilon) R_{*}} \cdot \prod_{\substack{j \leq k<\ell \\
\sigma^{k R_{*}}(\omega) \in \Omega_{B}}} \Gamma\left(\sigma^{k R_{*}}(\omega)\right) \\
& \leq\left(B_{*} e^{-(\xi-\varepsilon) R_{*}}\right)^{(1-\gamma)(\ell-j)} \cdot \exp \left(\left(\varepsilon^{\frac{1}{2}} \rho R_{*}\right)(\ell-j)\right) \\
& =\left(B_{*}^{1-\gamma} \exp \left(\left(\varepsilon^{\frac{1}{2}} \rho-(\xi-\varepsilon)(1-\gamma)\right) R_{*}\right)\right)^{\ell-j} \\
& <\left(B_{*} \exp \left(\left(\varepsilon^{\frac{1}{2}} \rho-(\xi-\varepsilon)(1-\gamma)\right) R_{*}\right)\right)^{\ell-j} .
\end{aligned}
$$


Now, since $B_{*}, \Gamma(\omega) \geq 1$ for all $\omega \in \Omega$, using (7.23) and (7.24), we have that for $0 \leq j<\ell$

$$
D_{\sigma^{j R_{*}}(\omega)}^{\left(R_{*}\right)} \leq B_{*} \Gamma\left(\sigma^{j R_{*}}(\omega)\right) \leq B_{*} \cdot \prod_{\substack{j \leq k<\ell \\ \sigma^{k R_{*}}(\omega) \in \Omega_{B}}} \Gamma\left(\sigma^{k R_{*}}(\omega)\right) \leq B_{*}\left(e^{\frac{1}{\varepsilon} \rho R_{*}}\right)^{(\ell-j)} .
$$

Thus, inserting (7.26) and (7.27) into (7.21) we see that

$$
\begin{aligned}
\operatorname{var}\left(\tilde{\mathcal{L}}_{\omega}^{\ell R_{*}} f\right) \leq & \left(\prod_{j=0}^{\ell-1} \Phi_{\sigma^{j R_{*}}(\omega)}^{\left(R_{*}\right)}\right) \operatorname{var}(f)+\sum_{j=0}^{\ell-1}\left(D_{\sigma^{j R_{*}}(\omega)}^{\left(R_{*}\right)} \cdot \prod_{k=j+1}^{\ell-1} \Phi_{\sigma^{k R_{*}}(\omega)}^{\left(R_{*}\right)}\right) \nu_{\omega}(f) \\
\leq & \left.B_{*} \exp \left(\left(\varepsilon^{\frac{1}{2}} \rho-(\xi-\varepsilon)(1-\gamma)\right) R_{*}\right)\right)^{\ell} \operatorname{var}(f) \\
& \quad+\nu_{\omega}(f) \sum_{j=0}^{\ell-1} B_{*}\left(e^{\varepsilon^{\frac{1}{2}} \rho R_{*}}\right)^{(\ell-j)} \cdot\left(B_{*} \exp \left(\left(\varepsilon^{\frac{1}{2}} \rho-(\xi-\varepsilon)(1-\gamma)\right) R_{*}\right)\right)^{\ell-j-1} \\
= & \left.B_{*} \exp \left(\left(\varepsilon^{\frac{1}{2}} \rho-(\xi-\varepsilon)(1-\gamma)\right) R_{*}\right)\right)^{\ell} \operatorname{var}(f) \\
& \quad+B_{*} e^{\frac{1}{2} \rho R_{*}} \cdot \nu_{\omega}(f) \sum_{j=0}^{\ell-1}\left(B_{*} \exp \left(\left(2 \varepsilon^{\frac{1}{2}} \rho-(\xi-\varepsilon)(1-\gamma)\right) R_{*}\right)\right)^{\ell-j-1}
\end{aligned}
$$

Therefore, using (7.19) in conjunction with (G1), we have that

$$
\begin{aligned}
\operatorname{var}\left(\mathcal{L}_{\omega}^{\ell R_{*}} f\right) & \leq\left(B_{*} e^{-\frac{\xi}{2} R_{*}}\right)^{\ell} \operatorname{var}(f)+B_{*} e^{\varepsilon^{\frac{1}{2}} \rho R_{*}} \cdot \nu_{\omega}(f) \sum_{j=0}^{\ell-1}\left(B_{*} e^{-\frac{\xi}{2} R_{*}}\right)^{\ell-j-1} \\
& \leq\left(\frac{1}{3}\right)^{\ell} \operatorname{var}(f)+B_{*} e^{\frac{1}{2} \rho R_{*}} \cdot \nu_{\omega}(f) \sum_{j=0}^{\ell-1}\left(\frac{1}{3}\right)^{\ell-j-1}
\end{aligned}
$$

and so we must have that

$$
\operatorname{var}\left(\tilde{\mathcal{L}}_{\omega}^{\ell R_{*}} f\right) \leq \frac{1}{3} \operatorname{var}(f)+2 B_{*} e^{\varepsilon^{\frac{1}{2}} \rho R_{*}} \nu_{\omega}(f) .^{7}
$$

Thus, for any $f \in \mathscr{C}_{\omega, a_{*}}$ we have that

$$
\operatorname{var}\left(\mathcal{L}_{\omega}^{\ell R_{*}} f\right) \leq \frac{a_{*}}{3} \nu_{\omega}(f)+\frac{a_{*}}{3} \nu_{\omega}(f)
$$

and consequently we have

$$
\tilde{\mathcal{L}}_{\omega}^{\ell R_{*}}\left(\mathscr{C}_{\omega, a_{*}}\right) \subseteq \mathscr{C}_{\sigma^{\ell R_{*}(\omega), 2 a_{*} / 3} \subseteq} \subseteq \mathscr{C}_{\sigma^{\ell R_{*}}(\omega), a_{*}}
$$

as desired.

The next lemma shows that the total length of the bad blocks take up only a small proportion of an orbit, however before stating the result we establish the following notation. For each $n \in \mathbb{N}$ we let $K_{n} \geq 0$ be the integer such that

$$
n=K_{n} R_{*}+\zeta(n)
$$

\footnotetext{
${ }^{7}$ Our final estimate of Lemma 7.5 differs from Buzzi's [10, Lemma 3.4] because of a term in the sum that was overlooked. The problem can be corrected by carefully choosing $R_{*}=R_{a_{0}}$ and then by defining $a_{*} \geq a_{0}$ in terms of $R_{*}$ as we have done here.
} 
where $0 \leq \zeta(n)<R_{*}$ is a remainder term. Given $\omega_{0} \in \Omega$, let

$$
\omega_{j}=\sigma^{\ell\left(\omega_{j-1}\right) R_{*}}\left(\omega_{j-1}\right)
$$

for each $j \geq 1$. Then for each $n \in \mathbb{N}$ we can break the $n$-length $\sigma$-orbit of $\omega_{0}$ in $\Omega$ into $k_{\omega_{0}}(n)$ blocks of length $\ell\left(\omega_{j}\right) R_{*}$ (for $0 \leq j \leq k_{\omega_{0}}(n)$ ) plus some remaining block of length $r_{\omega_{0}}(n) R_{*}$ where $0 \leq r_{\omega_{0}}(n)<\ell\left(\omega_{k_{\omega_{0}}(n)+1}\right)$ plus a remainder segment of length $\zeta(n)$, i.e. we can write

$$
n=\sum_{0 \leq j \leq k_{\omega_{0}}(n)} \ell\left(\omega_{j}\right) R_{*}+r_{\omega_{0}}(n) R_{*}+\zeta(n) ;
$$

see Figure 1. We also note that (7.28) and (7.30) imply that

$$
K_{n}=\sum_{0 \leq j \leq k_{\omega_{0}}(n)} \ell\left(\omega_{j}\right)+r_{\omega_{0}}(n) \text {. }
$$

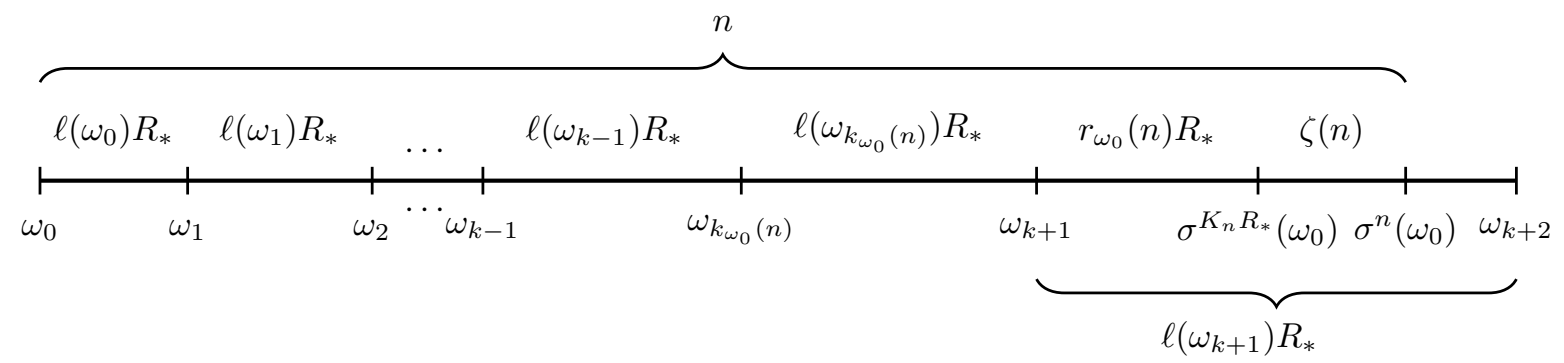

Figure 1. The decomposition of $n=\sum_{0 \leq j \leq k_{\omega_{0}}(n)} \ell\left(\omega_{j}\right) R_{*}+r_{\omega_{0}}(n) R_{*}+\zeta(n)$ and the fibers $\omega_{j}$.

Lemma 7.6. There exists a measurable function $N_{0}: \Omega \rightarrow \mathbb{N}$ such that for all $n \geq N_{0}\left(\omega_{0}\right)$ and for $m$-a.e. $\omega_{0} \in \Omega$ with $y_{*}\left(\omega_{0}\right)=0$ we have

$$
E_{\omega_{0}}(n):=\sum_{\substack{0 \leq j \leq k_{\omega_{0}}(n) \\ \omega_{j} \in \Omega_{B}}} \ell\left(\omega_{j}\right)+r_{\omega_{0}}(n)<H \cdot \varepsilon K_{n} \leq \frac{H}{R_{*}} \varepsilon n
$$

where

$$
H=H_{\varepsilon}:=\frac{2\left((2+\rho) R_{*}\right)}{\varepsilon^{\frac{1}{2}} \rho R_{*}},
$$

and where $K_{n}$ is as in (7.28), $\omega_{j}$ is as in (7.29), and $k_{\omega_{0}}(n)$ and $r_{\omega_{0}}(n)$ are as in (7.30).

Proof. Using (7.8), we see that for any $\omega \in \Omega_{B}$ and $2 \leq m \leq \ell(\omega)$ then $m-1 \geq m / 2$, and thus

$$
\sum_{0 \leq j<m}\left(\mathbb{1}_{\Omega_{B}} \log \Gamma\right)\left(\sigma^{j R_{*}}(\omega)\right)>\left(\varepsilon^{\frac{1}{2}} \rho R_{*}\right)(m-1) \geq \frac{m}{2}\left(\varepsilon^{\frac{1}{2}} \rho R_{*}\right) .
$$

Now, in view of Remark 7.2, we suppose that $\omega_{0} \in \Omega$ with $y_{*}\left(\omega_{0}\right)=0$ and $\ell\left(\omega_{j}\right)<\infty$ for each $j \geq 0$. Writing $n=K_{n} R_{*}+\zeta(n)$ and using (7.31) and (7.33) we have

$$
\sum_{0 \leq j<K_{n}}\left(\mathbb{1}_{\Omega_{B}} \log \Gamma\right)\left(\sigma^{j R_{*}}\left(\omega_{0}\right)\right)=\sum_{\substack{0 \leq j \leq k_{\omega_{0}}(n) \\ \omega_{j} \in \Omega_{B}}} \log \Gamma\left(\omega_{j}\right)+\sum_{\substack{0 \leq j<r_{\omega_{0}}(n) \\ \sigma^{j R *}\left(\omega_{k_{\omega_{0}}(n)}\right) \in \Omega_{B}}} \log \Gamma\left(\sigma^{j R_{*}}\left(\omega_{k_{\omega_{0}}(n)}\right)\right)
$$




$$
\begin{aligned}
& >\sum_{\substack{0 \leq j \leq k_{\omega_{0}}(n) \\
\omega_{j} \in \Omega_{B}}}\left(\frac{\ell\left(\omega_{j}\right)}{2}\left(\varepsilon^{\frac{1}{2}} \rho R_{*}\right)\right)+\frac{r_{\omega_{0}}(n)}{2}\left(\varepsilon^{\frac{1}{2}} \rho R_{*}\right) \\
& =\frac{1}{2}\left(\varepsilon^{\frac{1}{2}} \rho R_{*}\right)\left(\sum_{\substack{0 \leq j \leq k_{\omega_{0}}(n) \\
\omega_{j} \in \Omega_{B}}} \ell\left(\omega_{j}\right)+r_{\omega_{0}}(n)\right) \\
& =\frac{1}{2}\left(\varepsilon^{\frac{1}{2}} \rho R_{*}\right) E_{\omega_{0}}(n) .
\end{aligned}
$$

Now using (7.34) and (7.16) for all $n$ sufficiently large, say $n \geq N_{0}\left(\omega_{0}\right)$, we can write

$$
\frac{1}{2}\left(\varepsilon^{\frac{1}{2}} \rho R_{*}\right) E_{\omega_{0}}(n)<\sum_{0 \leq j<K_{n}}\left(\mathbb{1}_{\Omega_{B}} \log \Gamma\right)\left(\sigma^{j R_{*}}\left(\omega_{0}\right)\right) \leq \varepsilon\left((2+\rho) R_{*}\right) K_{n}
$$

and thus we have

$$
E_{\omega_{0}}(n)<\frac{2\left((2+\rho) R_{*}\right)}{+\varepsilon^{\frac{1}{2}} \rho R_{*}} \varepsilon K_{n} \leq \frac{2\left((2+\rho) R_{*}\right)}{\left(\varepsilon^{\frac{1}{2}} \rho R_{*}\right) R_{*}} \varepsilon n
$$

as desired.

To end this section we note that $\varepsilon \cdot H_{\varepsilon} \rightarrow 0$ as $\varepsilon \rightarrow 0$. Thus, we let

$$
\varepsilon_{4}:=\min \left\{\varepsilon_{3}, \sup \left\{\varepsilon>0: \varepsilon \cdot H_{\varepsilon}<\frac{1}{4}\right\}\right\} \text {. }
$$

\section{InVARIANt FAmily of Measures}

In this section we adapt the methods of [33] and [10] to establish the existence of invariant measures on the fibers $X_{\omega}$ as well as some of their properties. We now present the main technical result from which all the rest of our convergence results follow. Set

$$
\Delta:=\Delta_{a_{*}}=2 \log \frac{C_{*}\left(3+a_{*}\right)}{\alpha_{*}}
$$

to be the diameter value defined in Lemma 6.3 for the cone parameter $a_{*}$ defined in Lemma 7.5, and set

$$
\varepsilon_{5}:=\min \left\{\varepsilon_{4}, \frac{\xi}{2(4+3 \xi)}\right\} .
$$

Lemma 8.1. Let $\varepsilon<\varepsilon_{5}$ and $V: \Omega \rightarrow(0, \infty)$ not necessarily measurable. Then there exists $\vartheta \in(0,1)$ and a (not necessarily measurable) function $N_{3}: \Omega \rightarrow \mathbb{N}$ such that for $m$-a.e. $\omega \in \Omega$, all $n \geq N_{3}(\omega)$, all $l \geq 0$, and all $|p| \leq n$ we have

$$
\Theta_{\sigma^{n+p}(\omega),+}\left(\tilde{\mathcal{L}}_{\sigma^{p}(\omega)}^{n} f, \tilde{\mathcal{L}}_{\sigma^{p-l}(\omega)}^{n+l} h\right) \leq \Delta \vartheta^{n}
$$

for all $f \in \mathscr{C}_{\sigma^{p}(\omega),+}$ with $\nu_{\sigma^{p}(\omega)}(f)=1$ and $\operatorname{var}(f) \leq e^{\varepsilon n} V(\omega)$, and all $h \in \mathscr{C}_{\sigma^{p-l}(\omega),+}$ with $\nu_{\sigma^{p-l}(\omega)}(h)=1$ and $\operatorname{var}(h) \leq e^{\varepsilon(n+l)} V(\omega)$. Furthermore, $\Delta$ and $\vartheta$ do not depend on $V$.

Proof. We begin by noting that by (5.2) for each $l \geq 0$ we have that $\tilde{\mathcal{L}}_{\sigma^{p-l}(\omega)}^{l} h \in \mathscr{C}_{\sigma^{p}(\omega),+}$ for each $h \in \mathscr{C}_{\sigma^{p-l}(\omega),+}$, and let

$$
h_{l}=\tilde{\mathcal{L}}_{\sigma^{p-l}(\omega)}^{l} h .
$$


Set $y_{*}=y_{*}\left(\sigma^{p}(\omega)\right)$ (defined in Section 7) and let $d_{*}=d_{*}\left(\sigma^{p}(\omega)\right) \geq 0$ be the smallest integer that satisfies

$$
\begin{aligned}
& y_{*}+d_{*} R_{*} \geq \frac{\varepsilon n+\log V(\omega)}{\xi-\varepsilon}, \\
& C_{\varepsilon}\left(\sigma^{p+y_{*}+d_{*} R_{*}}(\omega)\right) \leq B_{*} .
\end{aligned}
$$

where $\xi$ was defined in (4.7). Choose

$$
N_{1}(\omega) \geq \frac{\log V(\omega)}{\varepsilon}
$$

and let $n \geq N_{1}(\omega)$. Now using (8.5) to write

$$
\frac{4 \varepsilon n}{\xi}=\frac{\varepsilon n+\varepsilon n}{\xi / 2} \geq \frac{\varepsilon n+\log V(\omega)}{\xi / 2}
$$

and then using (6.2) and (7.1), we see that (8.3) is satisfied for any $d_{*} R_{*} \geq 4 \varepsilon n / \xi$. Using (7.3), the construction of $y_{*}$, and the ergodic decomposition of $\sigma^{R_{*}}$ following (7.3), we have for $m$-a.e. $\omega \in \Omega$ there is an infinite, increasing sequence of integers $d_{j} \geq 0$ satisfying (8.4). Furthermore, (7.3) implies that

$$
\lim _{n \rightarrow \infty} \frac{1}{n / R_{*}} \#\left\{0 \leq k<\frac{n}{R_{*}}: C_{\varepsilon}\left(\sigma^{ \pm k R_{*}+y_{*}(\omega)}(\omega)\right)>B_{*}\right\}<\varepsilon,
$$

and thus for $n \in \mathbb{N}$ sufficiently large (depending measurably on $\omega$ ), say $n \geq N_{2}(\omega) \geq N_{1}(\omega)$, we have that

$$
\#\left\{0 \leq k<\frac{n}{R_{*}}: C_{\varepsilon}\left(\sigma^{ \pm k R_{*}+y_{*}(\omega)}(\omega)\right)>B_{*}\right\}<\frac{\varepsilon n}{R_{*}} .
$$

Thus, combining (8.6) and (8.7), we see that the smallest integer $d_{*}$ satisfying (8.3) and (8.4) also satisfies

$$
d_{*} R_{*} \leq \frac{4 \varepsilon n}{\xi}+\varepsilon n=\left(\frac{4+\xi}{\xi}\right) \varepsilon n
$$

Let

$$
\hat{y}_{*}=y_{*}+d_{*} R_{*} .
$$

Now, we wish to examine the iteration of our operator cocycle along a collection $\Sigma R_{*}$ of

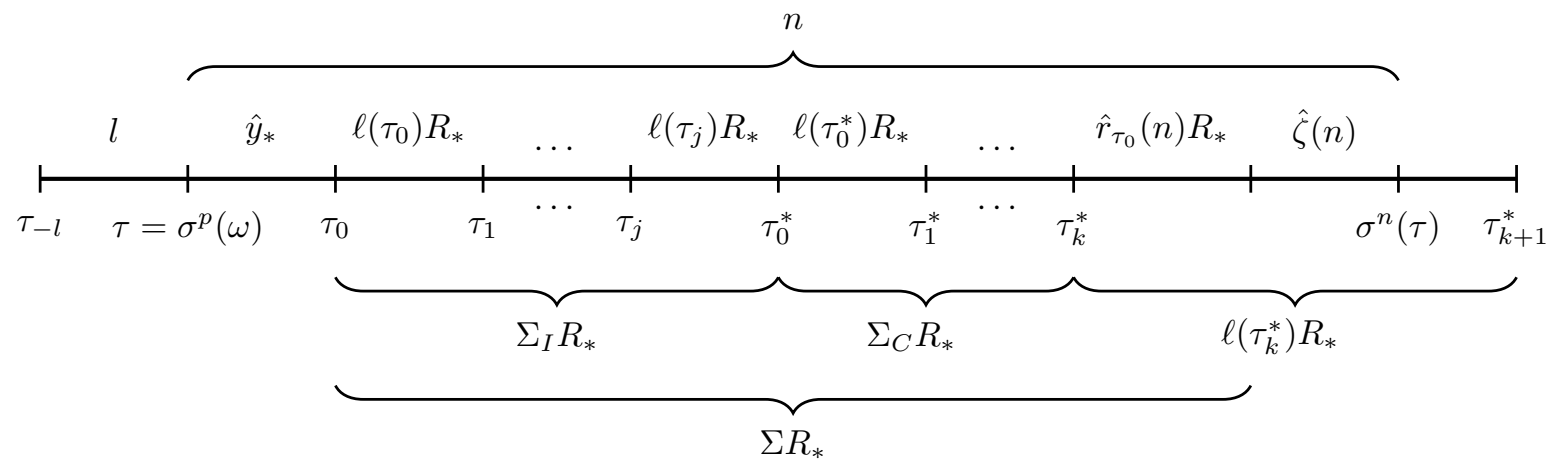

Figure 2. The fibers $\tau_{i}, \tau_{i}^{*}$ and the decomposition of $n=\hat{y}_{*}+\Sigma R_{*}+\hat{\zeta}(n)$. 
blocks, each of length $\ell(\omega) R_{*}$, so that the images of $\tilde{\mathcal{L}}_{\omega}^{\ell(\omega) R_{*}}$ are contained in $\mathscr{C}_{\sigma^{\ell(\omega) R_{*}}(\omega), a_{*}}$ as in Lemma 7.5. This collection $\Sigma R_{*}{ }^{8}$ will be comprised of an initial segment, $\Sigma_{I} R_{*}$, of bad blocks followed by a segment $\Sigma_{C} R_{*}$ of blocks, beginning with a good block, over which we obtain cone contraction with a finite diameter image, and finally a remainder segment, of length $\hat{r}_{\tau_{0}}(n) R_{*}$, which is not long enough to ensure cone contraction; see Figure 2.

We begin by establishing some simplifying notation. To that end, set $\tau=\sigma^{p}(\omega), \tau_{-l}=$ $\sigma^{p-l}(\omega)$, and $\tau_{0}=\sigma^{p+\hat{y}_{*}}(\omega)$; see Figure 2. Note that in light of (7.6) and (8.9) we have that

$$
y_{*}\left(\tau_{0}\right)=y_{*}\left(\sigma^{p+\hat{y}_{*}}(\omega)\right)=0 .
$$

Now, by our choice of $d_{*}$, we have that if $f \in \mathscr{C}_{\tau,+}$ with $\nu_{\tau}(f)=1$ and $\operatorname{var}(f) \leq e^{\varepsilon n} V(\omega)$, then

$$
\tilde{\mathcal{L}}_{\tau}^{\hat{y}_{*}} f \in \mathscr{C}_{\tau_{0}, a_{*}} .
$$

Indeed, applying Proposition 4.9 and (8.3) and (8.4) from above, we have

$$
\begin{aligned}
\operatorname{var}\left(\tilde{\mathcal{L}}_{\tau}^{\hat{y}_{*}} f\right) & \leq C_{\varepsilon}\left(\sigma^{\hat{y}_{*}}(\tau)\right) e^{-(\xi-\varepsilon) \hat{y}_{*}} \operatorname{var}(f)+C_{\varepsilon}\left(\sigma^{\hat{y}_{*}}(\tau)\right) \nu_{\tau}(f) \\
& \leq B_{*} e^{-(\xi-\varepsilon) \hat{y}_{*}} \operatorname{var}(f)+B_{*} \nu_{\tau}(f) \\
& \leq B_{*} \frac{\operatorname{var}(f)}{e^{\varepsilon n} V(\omega)}+B_{*} \leq 2 B_{*} \leq \frac{a_{*}}{3}
\end{aligned}
$$

where we recall that $a_{*}>6 B_{*}$ is defined in (7.20). A similar calculation yields that if $h \in \mathscr{C}_{\tau_{-l},+}$ with $\nu_{\tau_{-l}}(h)=1$ and $\operatorname{var}(h) \leq e^{\varepsilon(n+l)} V(\omega)$, then $\tilde{\mathcal{L}}_{\tau_{-l}}^{l+\hat{y}_{*}} h \in \mathscr{C}_{\tau_{0}, a_{*}}$.

Next, we wish to find the first good block; we denote the origin of this first good block by $\tau_{0}^{*}$. If $\tau_{0} \in \Omega_{G}$, then we are done, and we set $\tau_{0}=\tau_{0}^{*}$. However, it may be the case that $\tau_{0} \in \Omega_{B}$ and there may even be several bad blocks (originating at $\tau_{0}$ ) in a row before we encounter the first good block.

Now, if $\tau_{0} \in \Omega_{B}$, for each $i \geq 1$ while $\sigma^{\ell\left(\tau_{i-1}\right) R_{*}}\left(\tau_{i-1}\right) \in \Omega_{B}$ set $\tau_{i}=\sigma^{\ell\left(\tau_{i-1}\right) R_{*}}\left(\tau_{i-1}\right)$. Note that since $y_{*}\left(\tau_{0}\right)=0$, by Remark 7.2, we must have that $\ell\left(\tau_{i}\right)<\infty$ for each $i \geq 0$. Let $j \geq 1$ be the largest integer such that $\tau_{i} \in \Omega_{B}$ for each $1 \leq i \leq j$, and let

$$
\Sigma_{I}:=\sum_{i=0}^{j} \ell\left(\tau_{i}\right)
$$

be the total length of the initial coating intervals starting at $\tau_{0}$. Hence $\Sigma_{I} R_{*}$ is the total length of all the consecutive initial bad blocks; see Figure 2. Such an integer $j$ must exist for $m$-a.e. $\omega \in \Omega$ as Lemma 7.6 ensures that, for all $n \geq N_{1}(\omega) \geq N_{0}(\omega)$, we have

$$
\Sigma_{I} \leq E_{\tau_{0}}\left(n-\hat{y}_{*}\right) \leq E_{\tau_{0}}(n)<\frac{H}{R_{*}} \varepsilon n,
$$

and thus an initial segment of consecutive bad blocks can only take up a small portion of an orbit of length $n$. Therefore, we set $\tau_{0}^{*}=\sigma^{\ell\left(\tau_{j}\right) R_{*}}\left(\tau_{j}\right)$ to be the origin of the first good block after $\tau$, and for each $i \geq 1$ let $\tau_{i}^{*}=\sigma^{\ell\left(\tau_{i-1}^{*}\right) R_{*}}\left(\tau_{i-1}^{*}\right)$.

As there are only finitely many blocks (good and bad) that will occur within an orbit of length $n$, let $k \geq 1$ be the integer such that

$$
\hat{y}_{*}+\Sigma_{I} R_{*}+\sum_{i=0}^{k-1} \ell\left(\tau_{i}^{*}\right) R_{*} \leq n<\hat{y}_{*}+\Sigma_{I} R_{*}+\sum_{i=0}^{k} \ell\left(\tau_{i}^{*}\right) R_{*},
$$

\footnotetext{
${ }^{8}$ Here the number $\Sigma$ is playing the same role as the number $K_{n-\hat{y}_{*}}$ from Lemma 7.6.
} 
and let

$$
\Sigma_{C}:=\sum_{i=0}^{k-1} \ell\left(\tau_{i}^{*}\right) \quad \text { and } \quad \hat{r}_{\tau_{0}}(n):=r_{\tau_{0}}\left(n-\hat{y}_{*}\right)
$$

where $r_{\tau_{0}}\left(n-\hat{y}_{*}\right)$ is the number defined in (7.30). In other words, $\Sigma_{C} R_{*}$ is the total length of the consecutive good and bad blocks beginning with the first good block originating from $\tau_{0}^{*}$. Finally setting

$$
\Sigma=\Sigma_{I}+\Sigma_{C}+\hat{r}_{\tau_{0}}(n)^{9} \quad \text { and } \quad \hat{\zeta}(n):=n-\hat{y}_{*}-\Sigma R_{*},
$$

we have the right decomposition of our orbit length $n$ into blocks which do not expand distances in the fiber cones $\mathscr{C}_{\omega, a_{*}}$ and $\mathscr{C}_{\omega,+}$. Now let

$$
n \geq N_{3}(\omega):=\max \left\{N_{2}(\omega), \frac{R_{*}}{\varepsilon}\right\} .
$$

Since $y_{*}, \hat{\zeta}(n) \leq R_{*}$, by (8.13), (8.8), and our choice of $\varepsilon<\varepsilon_{5}$ (8.1), we must have that

$$
\begin{aligned}
\Sigma R_{*} & =n-\hat{y}_{*}-\hat{\zeta}(n)=n-y_{*}-d_{*} R_{*}-\hat{\zeta}(n) \\
& \geq n-\left(\frac{4+\xi}{\xi}\right) \varepsilon n-2 R_{*} \geq n-\left(\frac{4+\xi}{\xi}\right) \varepsilon n-2 \varepsilon n \\
& \geq n\left(1-\varepsilon\left(\frac{4+3 \xi}{\xi}\right)\right)>\frac{n}{2} .
\end{aligned}
$$

Now we note that since $\tilde{\mathcal{L}}_{\tau_{k}^{*}}^{\hat{\zeta}(n)}\left(\mathscr{C}_{\tau_{k}^{*},+}\right) \subseteq \mathscr{C}_{\sigma^{n+p}(\omega),+}$ we have that $\tilde{\mathcal{L}}_{\tau_{k}^{*}}^{\hat{\zeta}(n)}$ is a weak contraction, and hence, we have

$$
\Theta_{\sigma^{n+p}(\omega),+}\left(\tilde{\mathcal{L}}_{\tau_{k}^{*}}^{\hat{\zeta}(n)} f^{\prime}, \tilde{\mathcal{L}}_{\tau_{k}^{*}}^{\hat{\zeta}(n)} h^{\prime}\right) \leq \Theta_{\tau_{k}^{*},+}\left(f^{\prime}, h^{\prime}\right), \quad f^{\prime}, h^{\prime} \in \Theta_{\tau_{k}^{*},+} .
$$

Recall that $E_{\tau_{0}}\left(n-\hat{y}_{*}\right)$, defined in Lemma 7.6, is the total length of the coating intervals (bad blocks) of the $n-\hat{y}_{*}$ length orbit starting at $\tau_{0}$, i.e.

$$
E_{\tau_{0}}\left(n-\hat{y}_{*}\right)=\Sigma_{I}+\sum_{\substack{0 \leq j<k \\ \tau_{j}^{*} \in \Omega_{B}}} \ell\left(\tau_{j}^{*}\right)+r_{\tau_{0}}\left(n-\hat{y}_{*}\right) .
$$

Lemma 7.6 then gives that

$$
E_{\tau_{0}}\left(n-\hat{y}_{*}\right)<H \varepsilon \Sigma .
$$

We are now poised to calculate (8.2), but first we note that we can write

$$
\begin{aligned}
n & =\hat{y}_{*}+\Sigma R_{*}+\hat{\zeta}(n) \\
& =\hat{y}_{*}+\Sigma_{I} R_{*}+\Sigma_{C} R_{*}+\hat{\zeta}(n) \\
& =\hat{y}_{*}+\Sigma_{I} R_{*}+R_{*}+\left(\Sigma_{C}-1\right) R_{*}+\hat{\zeta}(n)
\end{aligned}
$$

and that the number of good blocks contained in the orbit of length $n-\hat{y}_{*}$ is given by

$$
\Sigma_{G}:=\#\left\{0 \leq j \leq k: \tau_{j}^{*} \in \Omega_{G}\right\}=\Sigma-E_{\tau_{0}}\left(n-\hat{y}_{*}\right) \leq \Sigma_{C} .
$$

\footnotetext{
${ }^{9}$ Note that if $\tau_{0} \in \Omega_{G}$, in which case we have $\tau_{0}=\tau_{0}^{*}$, then $\Sigma_{I}=0$ and $\Sigma=\Sigma_{+} \hat{r}_{\tau_{0}}(n)$.
} 
Now, using (8.17) we combine (in order) (8.15), (5.1), and Lemma 6.3 (repeatedly) in conjunction with the fact that $\tau_{0}^{*} \in \Omega_{G}$ to see that

$$
\begin{aligned}
& \Theta_{\sigma^{n+p}(\omega),+}\left(\tilde{\mathcal{L}}_{\tau}^{n}(f), \tilde{\mathcal{L}}_{\tau_{-l}}^{n+l}(h)\right) \\
& \quad=\Theta_{\sigma^{n+p}(\omega),+}\left(\tilde{\mathcal{L}}_{\tau_{k}^{*}}^{\hat{\zeta}(n)} \circ \tilde{\mathcal{L}}_{\tau_{0}}^{\Sigma R_{*}} \circ \tilde{\mathcal{L}}_{\tau}^{\hat{y}_{*}}(f), \tilde{\mathcal{L}}_{\tau_{k}^{*}}^{\hat{\zeta}(n)} \circ \tilde{\mathcal{L}}_{\tau_{0}}^{\Sigma R_{*}} \circ \tilde{\mathcal{L}}_{\tau}^{\hat{y}_{*}} \circ \tilde{\mathcal{L}}_{\tau_{-l}}^{l}(h)\right) \\
& \quad \leq \Theta_{\tau_{k}^{*},+}\left(\tilde{\mathcal{L}}_{\tau_{0}}^{\Sigma R_{*}} \circ \tilde{\mathcal{L}}_{\tau}^{\hat{y}_{*}}(f), \tilde{\mathcal{L}}_{\tau_{0}}^{\Sigma R_{*}} \circ \tilde{\mathcal{L}}_{\tau}^{\hat{y}_{*}}\left(h_{l}\right)\right) \\
& \quad \leq \Theta_{\tau_{k}^{*}, a_{*}}\left(\tilde{\mathcal{L}}_{\tau_{0}}^{\Sigma R_{*}} \circ \tilde{\mathcal{L}}_{\tau}^{\hat{y}_{*}}(f), \tilde{\mathcal{L}}_{\tau_{0}}^{\Sigma R_{*}} \circ \tilde{\mathcal{L}}_{\tau}^{\hat{y}_{*}}\left(h_{l}\right)\right) \\
& \quad=\Theta_{\tau_{k}^{*}, a_{*}}\left(\tilde{\mathcal{L}}_{\tau_{1}^{*}}^{\left(\Sigma_{C}-1\right) R_{*}} \circ \tilde{\mathcal{L}}_{\tau_{0}^{*}}^{R_{*}} \circ \tilde{\mathcal{L}}_{\tau_{0}}^{\Sigma_{I} R_{*}} \circ \tilde{\mathcal{L}}_{\tau}^{\hat{y}_{*}}(f), \tilde{\mathcal{L}}_{\tau_{1}^{*}}^{\left(\Sigma_{C}-1\right) R_{*}} \circ \tilde{\mathcal{L}}_{\tau_{0}^{*}}^{R_{*}} \circ \tilde{\mathcal{L}}_{\tau_{0}}^{\Sigma_{I} R_{*}} \circ \tilde{\mathcal{L}}_{\tau}^{\hat{y}_{*}}\left(h_{l}\right)\right) \\
& \quad \leq\left(\tanh \left(\frac{\Delta}{4}\right)\right)^{\Sigma_{G}-1} \Theta_{\tau_{1}^{*}, a_{*}}\left(\tilde{\mathcal{L}}_{\tau_{0}^{*}}^{R_{*}} \circ \tilde{\mathcal{L}}_{\tau_{0}}^{\Sigma_{I} R_{*}} \circ \tilde{\mathcal{L}}_{\tau}^{\hat{y}_{*}}(f), \tilde{\mathcal{L}}_{\tau_{0}^{*}}^{R_{*}} \circ \tilde{\mathcal{L}}_{\tau_{0}}^{\Sigma_{I} R_{*}} \circ \tilde{\mathcal{L}}_{\tau}^{\hat{y}_{*}}\left(h_{l}\right)\right) .
\end{aligned}
$$

Now since $\tau_{0}^{*} \in \Omega_{G}$ and since $\tilde{\mathcal{L}}_{\tau_{0}}^{\Sigma_{I} R_{*}} \circ \tilde{\mathcal{L}}_{\tau}^{\hat{y}_{*}}$ is a weak contraction (and thus non-expansive with respect to $\Theta_{\tau_{1}^{*}, a_{*}}$ ), in light of (8.11), applying Lemmas 6.3 and 7.5 gives that

$$
\Theta_{\tau_{1}^{*}, a_{*}}\left(\tilde{\mathcal{L}}_{\tau_{0}^{*}}^{R_{*}} \circ \tilde{\mathcal{L}}_{\tau_{0}}^{\Sigma_{I} R_{*}} \circ \tilde{\mathcal{L}}_{\tau}^{\hat{y}_{*}}(f), \tilde{\mathcal{L}}_{\tau_{0}^{*}}^{R_{*}} \circ \tilde{\mathcal{L}}_{\tau_{0}}^{\Sigma_{I} R_{*}} \circ \tilde{\mathcal{L}}_{\tau}^{\hat{y}_{*}}\left(h_{l}\right)\right) \leq \Delta .
$$

Using (8.18), the fact that $E_{\tau_{0}}\left(n-\hat{y}_{*}\right) \geq 1,(8.16)$, and (8.14), we see that

$$
\begin{aligned}
\Sigma_{G}-1 & =\Sigma-\left(E_{\tau_{0}}\left(n-\hat{y}_{*}\right)+1\right) \\
& \geq \Sigma-2 E_{\tau_{0}}\left(n-\hat{y}_{*}\right) \\
& \geq \Sigma-2 H \varepsilon \Sigma \\
& =\Sigma(1-2 H \varepsilon) \\
& \geq \frac{(1-2 H \varepsilon) n}{2 R_{*}} .
\end{aligned}
$$

Note that $1-2 H \varepsilon>0$ by our choice of $\varepsilon<\varepsilon_{5} \leq \varepsilon_{4}$ (7.35). Finally, inserting (8.20) and (8.21) into (8.19) gives

$$
\Theta_{\sigma^{n+p}(\omega),+}\left(\tilde{\mathcal{L}}_{\tau}^{n}(f), \tilde{\mathcal{L}}_{\tau_{-l}}^{n+l}(h)\right) \leq \Delta \vartheta^{n}
$$

where

which completes the proof.

$$
\vartheta:=\left(\tanh \left(\frac{\Delta}{4}\right)\right)^{\frac{(1-2 H \varepsilon)}{2 R_{*}}}<1
$$

Remark 8.2. Note that if $V: \Omega \rightarrow(0, \infty)$ is measurable then so is $d_{*}: \Omega \rightarrow \mathbb{N}$. Thus we may choose $N_{1}, N_{2}: \Omega \rightarrow \mathbb{N}$ measurable, which implies that $N_{3}: \Omega \rightarrow \mathbb{N}$ is also measurable. Also note that our choice of $N_{3}$ depends on $V$.

The following corollary establishes the existence of a family of fiberwise invariant (with respect to the normalized operator $\tilde{\mathcal{L}}_{\omega}$ ) densities (with respect to $\nu_{\omega}$ ).

Corollary 8.3. There exists a family of functions $\left(q_{\omega}\right)_{\omega \in \Omega}$ with $q_{\omega} \in \mathrm{BV}\left(X_{\omega}\right)$ and $\nu_{\omega}\left(q_{\omega}\right)=1$ for $m$-a.e. $\omega \in \Omega$ such that

$$
\mathcal{L}_{\omega} q_{\omega}=\lambda_{\omega} q_{\sigma(\omega)}
$$


Proof. Let $\varepsilon<\varepsilon_{5}$, and let $f \in \mathrm{BV}(I)$ with $f \geq 0$ such that $\nu_{\omega}\left(f_{\omega}\right)=1$ for $m$-a.e. $\omega \in \Omega$. Take $V(\omega)=\operatorname{var}(f)$. Then $f_{\omega} \in \mathscr{C}_{\omega,+}$ for each $\omega \in \Omega$. Setting

$$
f_{\omega, n}:=\tilde{\mathcal{L}}_{\sigma^{-n}(\omega)}^{n} f_{\sigma^{-n}(\omega)}
$$

for each $n \geq 0$, we note that Lemma 8.1 (with $p=-n$ ) and Lemma 5.5 (with $\varrho=\nu_{\sigma^{p+n}}(\omega)$ and $\left.\|\cdot\|=\|\cdot\|_{\infty}\right)$ together imply that the sequence $\left(f_{\omega, n}\right)_{n \geq 0}$ is Cauchy in $\mathscr{C}_{\omega,+}$. Indeed, we have

$$
\left\|f_{\omega, n}-f_{\omega, n+l}\right\|_{\infty} \leq\left\|f_{\omega, n}\right\|_{\infty}\left(e^{\Delta \vartheta^{n}}-1\right)
$$

for each $n \geq N_{3}(\omega)$ and $l \geq 0$. Thus, there must exist some $q_{\omega, f} \in \mathscr{C}_{\omega,+} \subseteq \operatorname{BV}\left(X_{\omega}\right)$ such that

$$
\lim _{n \rightarrow \infty} f_{\omega, n}=q_{\omega, f}
$$

Since $\nu_{\omega}\left(f_{\omega}\right)=1$ for $m$-a.e. $\omega \in \Omega$ we must also have that $\nu_{\omega}\left(q_{\omega, f}\right)=1$ for $m$-a.e. $\omega \in \Omega$. ${ }^{10}$

Now, to see that the limit in (8.22) does not depend on our choice of $f$, we suppose that $h \in \mathrm{BV}(I)$ with $\nu_{\omega}\left(h_{\omega}\right)=1$ for $m$-a.e. $\omega \in \Omega$ and $V(\omega)=\operatorname{var}(h)$. Then, as before we obtain

$$
q_{\omega, h}=\lim _{n \rightarrow \infty} h_{\omega, n}
$$

Using Lemma 8.1 again, we get

$$
\Theta_{\omega,+}\left(q_{\omega, f}, q_{\omega, h}\right) \leq \Theta_{\omega,+}\left(q_{\omega, f}, f_{\omega, n}\right)+\Theta_{\omega,+}\left(f_{\omega, n}, h_{\omega, n}\right)+\Theta_{\omega,+}\left(q_{\omega, h}, h_{\omega, n}\right) \leq 3 \Delta \vartheta^{n}
$$

for each $n \geq N_{3}(\omega)$. Inserting (8.23) into Lemma 5.5 with $\varrho=\nu_{\sigma^{p+n}(\omega)}$ and $\|\cdot\|=\|\cdot\|_{\infty}$ then gives

$$
\begin{aligned}
\left\|q_{\omega, f}-q_{\omega, h}\right\|_{\infty} & \leq\left(e^{\Theta_{\omega,+}\left(q_{\omega, f}, q_{\omega, h}\right)}-1\right)\left\|q_{\omega, f}\right\|_{\infty} \\
& \leq\left(e^{3 \Delta \vartheta^{n}}-1\right)\left\|q_{\omega, f}\right\|_{\infty},
\end{aligned}
$$

which goes to zero exponentially fast. But this of course implies that $q_{\omega, f}=q_{\omega, h}$ for $m$-a.e. $\omega \in \Omega$, and thus we denote their common value by $q_{\omega}$. In particular, (8.22) gives that

$$
\tilde{\mathcal{L}}_{\omega} q_{\omega}=\tilde{\mathcal{L}}_{\omega}\left(\lim _{n \rightarrow \infty} \tilde{\mathcal{L}}_{\sigma^{-n}(\omega)}^{n} \mathbb{1}_{\sigma^{-n}(\omega)}\right)=\lim _{n \rightarrow \infty} \tilde{\mathcal{L}}_{\sigma^{-n}(\omega)}^{n+1} \mathbb{1}_{\sigma^{-n}(\omega)}=q_{\sigma(\omega)} .
$$

Remark 8.4. The proof of the previous Lemma shows that $q_{\omega} \in \mathrm{BV}\left(X_{\omega}\right)$ for each $\omega \in \Omega$. Furthermore, we note that it follows from Proposition 4.9 and (8.24) that

$$
\operatorname{var}\left(q_{\omega}\right) \leq C_{\varepsilon}(\omega)
$$

Indeed, for each $n \in \mathbb{N}$, Proposition 4.9 gives

$$
\operatorname{var}\left(\tilde{\mathcal{L}}_{\sigma^{-n}(\omega)}^{n} \mathbb{1}_{\sigma^{-n}(\omega)}\right) \leq C_{\varepsilon}(\omega) e^{-(\xi-\varepsilon) n} \operatorname{var}\left(\mathbb{1}_{\sigma^{-n}(\omega)}\right)+C_{\varepsilon}(\omega),
$$

and in light of (8.24), we must in fact have (8.25).

Using (8.25) we get that

$$
\left\|q_{\omega}\right\|_{\mathrm{BV}}=\left\|q_{\omega}\right\|_{\infty}+\operatorname{var}\left(q_{\omega}\right) \leq 2 \operatorname{var}\left(q_{\omega}\right)+\nu_{\omega}\left(q_{\omega}\right) \leq 2 C_{\varepsilon}(\omega)+1 .
$$

The following lemma shows that the BV norm of the invariant density $q_{\omega}$ does not grow too much along a $\sigma$-orbit of fibers by providing a measurable upper bound.

\footnotetext{
${ }^{10}$ Indeed, one can see this by considering the continuous linear functional $\Lambda_{\omega}$ which is equivalent to $\nu_{\omega}$ on $\mathrm{BV}(I)$. As $f_{\omega, n} \rightarrow q_{\omega, f}$ we must have $1=\Lambda_{\omega}\left(f_{\omega, n}\right) \rightarrow \Lambda\left(q_{\omega, f}\right)=\nu_{\omega}\left(q_{\omega, f}\right)=1$.
} 
Lemma 8.5. For all $\delta>0$ there exists a measurable random constant $C(\omega, \delta)>0$ such that for all $k \in \mathbb{Z}$ and $m$-a.e. $\omega \in \Omega$ we have

$$
\left\|q_{\sigma^{k}(\omega)}\right\|_{\mathrm{BV}}=\left\|q_{\sigma^{k}(\omega)}\right\|_{\infty}+\operatorname{var}\left(q_{\sigma^{k}(\omega)}\right) \leq C(\omega, \delta) e^{\delta|k|} .
$$

Proof. Let $\varepsilon<\varepsilon_{5}$ and let $0<\delta<2\left(\rho_{0}+1\right)$ where

$$
\rho_{0}:=\int_{\Omega} \log L_{\omega} d m(\omega) .
$$

By the ergodic theorem for $m$-a.e. $\omega \in \Omega$ there exists a measurable $n_{\delta}(\omega) \in \mathbb{N}$ such that for all $n \geq n_{\delta}(\omega)$ and each choice of sign + or - , we have

$$
\sum_{j=0}^{n-1} \log L_{\sigma^{ \pm j}(\omega)} \leq\left(\rho_{0}+1\right) n .
$$

Now let $n_{*} \in \mathbb{N}$ so large that

$$
m\left(\left\{\omega \in \Omega: n_{\delta}(\omega)>n_{*}\right\}\right)<\frac{\delta}{32\left(\rho_{0}+1\right)} .
$$

By (8.25) we have that $\operatorname{var}\left(q_{\omega}\right) \leq C_{\varepsilon}(\omega)<\infty$ for $m$-a.e. $\omega \in \Omega$, and so for $A_{\delta} \geq 1$ sufficiently large we have

$$
m\left(\left\{\omega \in \Omega: C_{\varepsilon}(\omega)>A_{\delta}\right\}\right)<\frac{\delta}{32\left(\rho_{0}+1\right)} .
$$

Thus the ergodic theorem gives that for $m$-a.e. $\omega \in \Omega$ we have

$$
\lim _{n \rightarrow \pm \infty} \frac{1}{|n|} \#\left\{0 \leq j<|n|: C_{\varepsilon}\left(\sigma^{ \pm j}(\omega)\right)>A_{\delta} \text { or } n_{\delta}\left(\sigma^{ \pm j}(\omega)\right)>n_{*}\right\}<\frac{\delta}{8\left(\rho_{0}+1\right)}<1 .
$$

Then for $m$-a.e. $\omega \in \Omega$ and all large $2|k|$ (depending on $\omega$ ) there exists an integer

$$
p \in\left(k-\frac{\delta|k|}{2\left(\rho_{0}+1\right)}, k-\frac{\delta|k|}{4\left(\rho_{0}+1\right)}\right)
$$

such that

$$
\operatorname{var}\left(q_{\sigma^{p}(\omega)}\right) \leq C_{\varepsilon}\left(\sigma^{p}(\omega)\right) \leq A_{\delta} \quad \text { and } \quad n_{\delta}\left(\sigma^{p}(\omega)\right) \leq n_{*}
$$

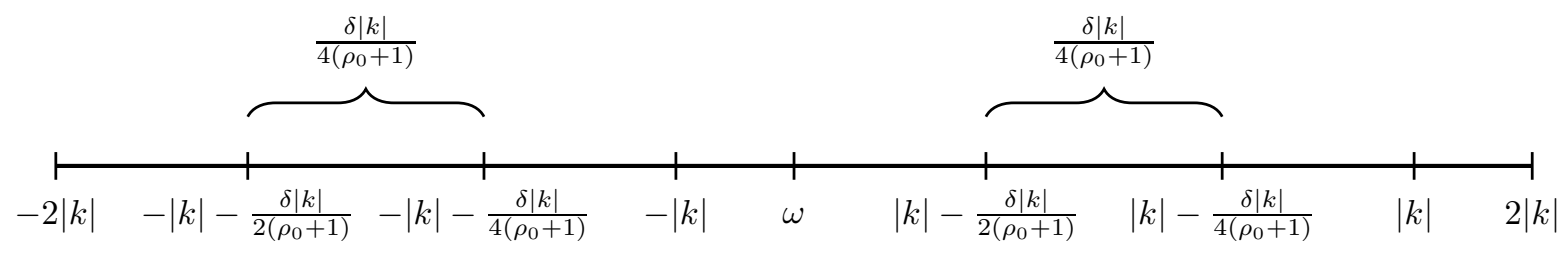

FIGURE 3. The $2|k|$ length forward and backward $\sigma$-orbits originating at $\omega$.

Without loss of generality we assume that

$$
|k| \geq \frac{4 n_{*}\left(\rho_{0}+1\right)}{\delta} .
$$

Now we note that since $k>p$, we have that

$$
0<k-p<|k|
$$


for each $k \in \mathbb{Z}$ sufficiently large. Furthermore, using (8.29) and (8.31) we have that

$$
n_{*} \leq \frac{\delta|k|}{4\left(\rho_{0}+1\right)}<k-p<\frac{\delta|k|}{2\left(\rho_{0}+1\right)}<|k| \frac{\delta}{2} .
$$

Now using (8.33) and the fact that $x<e^{x}$ for $x>0$ gives that

$$
A_{\delta}+k-p<A_{\delta}+|k| \frac{\delta}{2}<A_{\delta} \cdot|k| \frac{\delta}{2}<A_{\delta} e^{|k| \frac{\delta}{2}} .
$$

Using, in order, (8.24) and (4.8) (with $n=1$ ) repeatedly followed by (8.30) and (8.34), we see that, for all large $|k|$, say $|k| \geq k_{\delta}(\omega) \geq 1$ with $k_{\delta}: \Omega \rightarrow \mathbb{N}$ measurable and satisfying (8.29) and (8.31), (8.27) gives that

$$
\begin{aligned}
\left\|q_{\sigma^{k}(\omega)}\right\|_{\mathrm{BV}} & =\left\|\tilde{\mathcal{L}}_{\sigma^{p}(\omega)}^{k-p} q_{\sigma^{p}(\omega)}\right\|_{\mathrm{BV}} \\
& =\operatorname{var}\left(\tilde{\mathcal{L}}_{\sigma^{k-1}} \circ \cdots \circ \tilde{\mathcal{L}}_{\sigma^{p}(\omega)}\left(q_{\sigma^{p}(\omega)}\right)\right)+\left\|\tilde{\mathcal{L}}_{\sigma^{k-1}} \circ \cdots \circ \tilde{\mathcal{L}}_{\sigma^{p}(\omega)}\left(q_{\sigma^{p}(\omega)}\right)\right\|_{\infty} \\
& \leq L_{\sigma^{k-1}(\omega)}\left(\operatorname{var}\left(\tilde{\mathcal{L}}_{\sigma^{k-2}} \circ \cdots \circ \tilde{\mathcal{L}}_{\sigma^{p}(\omega)}\left(q_{\sigma^{p}(\omega)}\right)\right)+1\right) \\
& \leq \prod_{j=0}^{k-p-1} L_{\sigma^{p+j}(\omega)} \operatorname{var}\left(q_{\sigma^{p}(\omega)}\right)+\sum_{j=0}^{k-p-1} \prod_{i=j}^{k-p-1} L_{\sigma^{p+i}(\omega)} \\
& \leq\left(A_{\delta}+k-p\right) \prod_{j=0}^{k-p-1} L_{\sigma^{p+j}(\omega)} \\
& <A_{\delta} e^{|k| \frac{\delta}{2}} \prod_{j=0}^{k-p-1} L_{\sigma^{p+j}(\omega)} \leq A_{\delta} e^{|k| \frac{\delta}{2}} e^{(k-p)\left(\rho_{0}+1\right)} \leq A_{\delta} e^{|k| \frac{\delta}{2}} e^{|k| \frac{\delta}{2}}=A_{\delta} e^{|k| \delta} .
\end{aligned}
$$

To obtain the inequality (8.36) for all $k \in \mathbb{Z}$, and not just $|k| \geq k_{\delta}(\omega)$, we use (8.26) to define the measurable constant

$$
C(\omega, \delta):=\max _{|k|<k_{\delta}(\omega)}\left\{2 C_{\varepsilon}\left(\sigma^{k}(\omega)\right)+1\right\} .
$$

Thus combining with (8.36), we have

$$
\left\|q_{\sigma^{k}(\omega)}\right\|_{\mathrm{BV}} \leq C(\omega, \delta) e^{|k| \delta}
$$

for all $k \in \mathbb{Z}$, which finishes the proof.

Definition 8.6. Given $f \in \prod_{\omega \in \Omega} \mathrm{BV}(I), \omega \in \Omega$, and $n \in \mathbb{Z}$ we will say that $(\mathcal{D} 1)$, respectively (D2), hold for the triple $(f, \omega, n)$ if for each $\varepsilon>0$ there exists $V_{f, \varepsilon}: \Omega \rightarrow(0, \infty)$ such that the following respective conditions hold:

$(\mathcal{D} 1) \operatorname{var}\left(f_{\sigma^{n}(\omega)}\right) \leq V_{f, \varepsilon}(\omega) e^{\varepsilon|n|}$,

(D2) $\nu_{\sigma^{n}(\omega)}\left(\left|f_{\sigma^{n}(\omega)}\right|\right) \geq V_{f, \varepsilon}^{-1}(\omega) e^{-\varepsilon|n|}$.

We let $\mathcal{D}$ denote the collections of functions $f \in \prod_{\omega \in \Omega} \mathrm{BV}(I)$ such that (D1) and (D2) hold for the triple $(f, \omega, n)$ for $m$-a.e. $\omega \in \Omega$ and each $n \in \mathbb{Z}$. Let $\mathcal{D}^{+} \subseteq \mathcal{D}$ denote the collection of all functions $f \in \mathcal{D}$ such that $f_{\omega} \geq 0$ for each $\omega \in \Omega$. 
Remark 8.7. Note that $\mathcal{D}^{+}$is nonempty as it contains the functions $\mathbb{1}$ and $q$ by Lemma 8.5. Furthermore, note that $(\mathcal{D} 1)$ and $(\mathcal{D} 2)$ are clearly satisfied for all $f \in \mathrm{BV}_{\Omega}^{l}(I)$, which was defined in (2.3).

The following lemma provides conditions ensuring that $(\mathcal{D} 1)$ and (D2) hold for products and quotients. Its proof consists entirely of elementary calculations, and is therefore left to the reader.

Lemma 8.8. If $f \in \mathcal{D}$ then

(a) $\left(\frac{f_{\omega}}{\nu_{\omega}\left(f_{\omega}\right)}\right)_{\omega \in \Omega} \in \mathcal{D}$.

Furthermore, if $h \in \prod_{\omega \in \Omega} B V^{+}(I)$ and there exists a sequence $\left(n_{k}\right)_{k \in \mathbb{N}}$ such that

$$
\inf h_{\sigma^{n_{k}(\omega)}}>0 \quad \text { and } \quad \lim _{k \rightarrow \infty} \frac{1}{n_{k}} \log \inf h_{\sigma^{n_{k}(\omega)}}=0
$$

and $(\mathcal{D} 1)$ holds for $\left(h, \omega, n_{k}\right)$ for each $n_{k}$, then we have the following:

(b) (D1) and (D2) hold for $\left(f h, \omega, n_{k}\right)$ for each $n_{k}$,

(c) (D1) and (D2) hold for $\left(\frac{f}{h}, \omega, n_{k}\right)$ for each $n_{k}$.

We now wish to describe the rate at which the convergence to the invariant density $q_{\omega}$ from Corollary 8.3 is achieved.

Corollary 8.9. There exists $0<\kappa<1$ such that if $f \in \prod_{\omega \in \Omega} B V^{+}(I)$, with $\nu_{\omega}\left(f_{\omega}\right)=1$ for $m$-a.e. $\omega \in \Omega$ then there exists $A_{f}(\omega): \Omega \rightarrow(0, \infty)$ such that for all $n \in \mathbb{N}$ and all $|p| \leq n$, with (D1) holding for $\left(f, \sigma^{p}(\omega), n\right)$, we have

$$
\left\|\tilde{\mathcal{L}}_{\sigma^{p}(\omega)}^{n} f_{\sigma^{p}(\omega)}-q_{\sigma^{p+n}(\omega)}\right\|_{\infty} \leq A_{f}(\omega)\left\|f_{\sigma^{p}(\omega)}\right\|_{\mathrm{BV}} \kappa^{n} .
$$

Proof. We begin by noting that Lemma 4.6, together with the fact that $\left\|f_{\omega}\right\|_{\mathrm{BV}},\left\|q_{\omega}\right\|_{\infty} \geq 1$ for $m$-a.e. $\omega \in \Omega$, implies that for each $n \in \mathbb{N}$ we have

$$
\begin{aligned}
\left\|\tilde{\mathcal{L}}_{\sigma^{p}(\omega)}^{n} f_{\sigma^{p}(\omega)}-q_{\sigma^{p+n}(\omega)}\right\|_{\infty} & \leq\left\|\tilde{\mathcal{L}}_{\sigma^{p}(\omega)}^{n} f_{\sigma^{p}(\omega)}\right\|_{\infty}+\left\|q_{\sigma^{p+n}(\omega)}\right\|_{\infty} \\
& \leq 2 L_{\sigma^{p}(\omega)}^{n}\left\|f_{\sigma^{p}(\omega)}\right\|_{\mathrm{BV}}\left\|q_{\sigma^{p+n}(\omega)}\right\|_{\infty} \\
& \leq 2 L_{\sigma^{-n}(\omega)}^{3 n}\left\|f_{\sigma^{p}(\omega)}\right\|_{\mathrm{BV}}\left\|q_{\sigma^{p+n}(\omega)}\right\|_{\infty} .
\end{aligned}
$$

Now, fix $\varepsilon<\varepsilon_{5}$ small as in Lemma 8.1. Lemma 8.5 implies that

$$
\operatorname{var}\left(q_{\sigma^{p+n}(\omega)}\right) \leq C(\omega, \varepsilon) e^{\varepsilon|p+n|} .
$$

for all $n \geq 0$. Let

$$
V(\omega)=\max \left\{C(\omega, \varepsilon), V_{f, \varepsilon}(\omega)\right\} .
$$

Then Lemma 8.1 gives that

$$
\Theta_{\sigma^{p+n}(\omega),+}\left(\tilde{\mathcal{L}}_{\sigma^{p}(\omega)}^{n} f_{\sigma^{p}(\omega)}, q_{\sigma^{p+n}(\omega)}\right)=\Theta_{\sigma^{p+n}(\omega),+}\left(\tilde{\mathcal{L}}_{\sigma^{p}(\omega)}^{n} f_{\sigma^{p}(\omega)}, \tilde{\mathcal{L}}_{\sigma^{p}(\omega)}^{n} q_{\sigma^{p}(\omega)}\right) \leq \Delta \vartheta^{n}
$$


for all $n \geq N_{3}(\omega)$, which was defined in (8.13) in the proof of Lemma 8.1. Applying Lemma 5.5 with $\varrho=\nu_{\sigma^{p+n}(\omega)}$ and $\|\cdot\|=\|\cdot\|_{\infty}$, together with Lemmas 8.1 and 8.5 then gives

$$
\begin{aligned}
\left\|\tilde{\mathcal{L}}_{\sigma^{p}(\omega)}^{n} f_{\sigma^{p}(\omega)}-q_{\sigma^{p+n}(\omega)}\right\|_{\infty} & \leq\left\|q_{\sigma^{p+n}(\omega)}\right\|_{\infty}\left(\exp \left(\Theta_{\sigma^{p+n}(\omega),+}\left(\tilde{\mathcal{L}}_{\sigma^{p}(\omega)}^{n} f_{\sigma^{p}(\omega)}, q_{\sigma^{p+n}(\omega)}\right)\right)-1\right) \\
& \leq\left\|q_{\sigma^{p+n}(\omega)}\right\|_{\infty}\left(e^{\Delta \vartheta^{n}}-1\right) \leq\left\|q_{\sigma^{p+n}(\omega)}\right\|_{\infty} \tilde{\kappa}^{n}
\end{aligned}
$$

for some $\tilde{\kappa} \in(0,1)$. Combining (8.38) with (8.40) we see that

$$
\begin{aligned}
\left\|\tilde{\mathcal{L}}_{\sigma^{p}(\omega)}^{n} f_{\sigma^{p}(\omega)}-q_{\sigma^{p+n}(\omega)}\right\|_{\infty} & \leq 2 \max _{0 \leq j \leq N_{3}(\omega)}\left\{L_{\sigma^{p}(\omega)}^{j} \tilde{\kappa}^{-j}\right\}\left\|f_{\sigma^{p}(\omega)}\right\|_{\mathrm{BV}}\left\|q_{\sigma^{p+n}(\omega)}\right\|_{\infty} \tilde{\kappa}^{n} \\
& =2 L_{\sigma^{p}(\omega)}^{N_{3}(\omega)} \tilde{\kappa}^{-N_{3}(\omega)}\left\|f_{\sigma^{p}(\omega)}\right\|_{\mathrm{BV}}\left\|q_{\sigma^{p+n}(\omega)}\right\|_{\infty} \tilde{\kappa}^{n} \\
& \leq 2 L_{\sigma^{-N_{3}(\omega)}(\omega)}^{3 N_{3}(\omega)} \tilde{\kappa}^{-N_{3}(\omega)}\left\|f_{\sigma^{p}(\omega)}\right\|_{\mathrm{BV}}\left\|q_{\sigma^{p+n}(\omega)}\right\|_{\infty} \tilde{\kappa}^{n}
\end{aligned}
$$

for all $n \in \mathbb{N}$. Now in light of Lemma 8.5 we may rewrite (8.41) so that we have

$$
\left\|\tilde{\mathcal{L}}_{\sigma^{p}(\omega)}^{n} f_{\sigma^{p}(\omega)}-q_{\sigma^{p+n}(\omega)}\right\|_{\infty} \leq A_{f}(\omega)\left\|f_{\sigma^{p}(\omega)}\right\|_{\mathrm{BV}} \kappa^{n}
$$

for all $n \in \mathbb{N}$, where

$$
A_{f}(\omega):=2 C(\omega, \delta) L_{\sigma^{-N_{3}(\omega)}(\omega)}^{3 N_{3}(\omega)} \tilde{\kappa}^{-N_{3}(\omega)}
$$

and $\kappa:=\tilde{\kappa} e^{2 \delta}$ for some $\delta>0$ sufficiently small.

The following proposition serves two purposes: we show that the invariant density $q_{\omega}$ is strictly positive and finite for $m$-a.e. $\omega \in \Omega$, and we provide measurable upper and lower bounds for $q_{\omega}$ despite the fact that (at this point) we do not know whether the maps $\omega \mapsto \inf q_{\omega}$ or $\omega \mapsto\left\|q_{\omega}\right\|_{\infty}$ are measurable as we also do not currently know that the maps $\omega \mapsto \nu_{\omega}$ and $\omega \mapsto \lambda_{\omega}$ are measurable.

Proposition 8.10. There exist measurable functions $u, U: \Omega \rightarrow(0, \infty)$ such that for m-a.e. $\omega \in \Omega$ we have

$$
u(\omega) \leq q_{\omega} \leq U(\omega) .
$$

Proof. For the upper bound we simply set $U(\omega)=C(\omega, \delta)$ as in Lemma 8.5. Now, for the lower bound we begin by noting that (8.25), our choice of $B_{*}$ in the proof of Lemma 6.2, and the fact that we have $a_{*}>6 B_{*}$ gives that $q_{\omega} \in \mathscr{C}_{\omega, a_{*}}$ for each $\omega \in \Omega_{G}$. Thus, for $\omega \in \Omega_{G}$ (6.10) implies that

$$
\inf q_{\sigma_{*}^{R_{*}(\omega)}}=\inf \tilde{\mathcal{L}}_{\omega}^{R_{*}} q_{\omega} \geq \frac{1}{2} \inf \tilde{\mathcal{L}}_{\omega}^{R_{*}} \mathbb{1}_{U_{q_{\omega}}} \geq \frac{\alpha_{*}}{2 \lambda_{\omega}^{R_{*}}} \geq \frac{\alpha_{*}}{2 C_{*}}=: t_{*}>0,
$$

where $U_{q_{\omega}} \in \mathcal{U}_{\omega}$ is coming from Lemma 5.9, and thus

$$
\Omega_{+}:=\sigma^{R_{*}}\left(\Omega_{G}\right) \subseteq \mathcal{H}:=\left\{\omega \in \Omega: \inf q_{\omega}>0\right\} .
$$

In light of $(8.42)$, for $\omega \in \Omega_{+}$we have

$$
\inf q_{\omega} \geq t_{*}
$$

and (8.43) together with Lemma 6.2 imply that

$$
m\left(\Omega_{+}\right) \geq m\left(\Omega_{G}\right) \geq 1-\frac{\varepsilon}{4} .
$$


Note that for any $\omega \in \mathcal{H}$ (up to a set of measure zero) and any $n \in \mathbb{N}$ we have

$$
\begin{aligned}
\inf q_{\omega} & =\inf \tilde{\mathcal{L}}_{\sigma^{-n}(\omega)}^{n} q_{\sigma^{-n}(\omega)} \\
& \geq\left(\lambda_{\sigma^{-n}(\omega)}^{n}\right)^{-1} \inf q_{\sigma^{-n}(\omega)} \inf \mathcal{L}_{\sigma^{-n}(\omega)}^{n} \mathbb{1}_{\sigma^{-n}(\omega)} \\
& \geq \inf q_{\sigma^{-n}(\omega)} \frac{\inf \mathcal{L}_{\sigma^{-n}(\omega)}^{n} \mathbb{1}_{\sigma^{-n}(\omega)}}{\left\|\mathcal{L}_{\sigma^{-n}(\omega)}^{n} \mathbb{1}_{\sigma^{-n}(\omega)}\right\|_{\infty}}>0
\end{aligned}
$$

since Lemma 2.4 implies that the fraction on the right-hand side is positive for $m$-a.e. $\omega \in \Omega$. Now since $\Omega_{+}$has positive measure, the ergodic theorem ensures that we may define the measurable number

$$
\tilde{n}_{\omega}:=\min \left\{n \in \mathbb{N}: \sigma^{-n}(\omega) \in \Omega_{+}\right\} .
$$

Then, using (8.44), we have

$$
\begin{aligned}
\inf q_{\omega} & =\inf \tilde{\mathcal{L}}_{\sigma^{-\tilde{n}_{\omega}(\omega)}}^{\tilde{n}_{\omega}} q_{\sigma^{-\tilde{n}_{\omega}(\omega)}} \geq\left(\lambda_{\sigma^{-\tilde{n}_{\omega}(\omega)}}^{\tilde{n}_{\omega}}\right)^{-1} \inf q_{\sigma^{-\tilde{n}_{\omega}(\omega)}} \inf \mathcal{L}_{\sigma^{-\tilde{n}_{\omega}(\omega)}}^{\tilde{n}_{\omega}} \mathbb{1}_{\sigma^{-\tilde{n}_{\omega}(\omega)}} \\
& \geq t_{*} \frac{\inf \mathcal{L}_{\sigma^{-\tilde{n}_{\omega}(\omega)}}^{\tilde{n}_{\omega}} \mathbb{1}_{\sigma^{-\tilde{n}_{\omega}(\omega)}}}{\left\|\mathcal{L}_{\sigma^{-\tilde{n}_{\omega}(\omega)}}^{\tilde{n}_{\omega}} \mathbb{1}_{\sigma^{-\tilde{n}_{\omega}(\omega)}}\right\|_{\infty}}>0
\end{aligned}
$$

Taking

$$
u(\omega)=t_{*} \cdot \frac{\inf \mathcal{L}_{\sigma^{-} \tilde{n}_{\omega}(\omega)}^{\tilde{n}_{\omega}} \mathbb{1}_{\sigma^{-\tilde{n} \omega}(\omega)}}{\left\|\mathcal{L}_{\sigma^{-\tilde{n}_{\omega}}(\omega)}^{\tilde{n}_{\omega}} \mathbb{1}_{\sigma^{-\tilde{n}_{\omega}(\omega)}}\right\|_{\infty}}
$$

finishes the proof.

For each $\omega \in \Omega$ we may now define the measure $\mu_{\omega} \in \mathcal{P}(I)$ by

$$
\mu_{\omega}(f):=\int_{X_{\omega}} f q_{\omega} d \nu_{\omega}, \quad f \in L_{\nu_{\omega}}^{1}(I)
$$

Proposition 3.1 and Proposition 8.10 together show that, for $m$-a.e. $\omega \in \Omega$, $\mu_{\omega}$ is nonatomic, positive on non-degenerate intervals, and absolutely continuous with respect to $\nu_{\omega}$. Furthermore, in view of Proposition 8.10, for $m$-a.e. $\omega \in \Omega$, we may now define the fully normalized transfer operator $\hat{\mathcal{L}}_{\omega}: \mathrm{BV}\left(X_{\omega}\right) \rightarrow \mathrm{BV}\left(X_{\sigma(\omega)}\right)$ by

$$
\hat{\mathcal{L}}_{\omega} f:=\frac{1}{q_{\sigma(\omega)}} \tilde{\mathcal{L}}_{\omega}\left(f q_{\omega}\right)=\frac{1}{\lambda_{\omega} q_{\sigma(\omega)}} \mathcal{L}_{\omega}\left(f q_{\omega}\right), \quad f \in \mathrm{BV}\left(X_{\omega}\right) .
$$

Alternatively, we may think of the fully normalized transfer operator $\hat{\mathcal{L}}_{\omega}$ as the usual transfer operator taken with the potential $\hat{\varphi}_{\omega}$ which is given by

$$
\hat{\varphi}_{\omega}:=\varphi_{\omega}+\log q_{\omega}-\log q_{\sigma(\omega)} \circ T_{\omega}-\log \lambda_{\omega},
$$

i.e. $\hat{\mathcal{L}}_{\omega}=\mathcal{L}_{\hat{\varphi}, \omega}$. This gives rise to the weight functions $\hat{g}_{\omega}$ given by

$$
\hat{g}_{\omega}:=e^{\hat{\varphi}_{\omega}}=\frac{e^{\varphi_{\omega}} \cdot q_{\omega}}{\lambda_{\omega} \cdot q_{\sigma(\omega)} \circ T_{\omega}},
$$


and the alternate formulation of $\hat{\mathcal{L}}_{\omega}$ :

$$
\hat{\mathcal{L}}_{\omega}(f)(x)=\sum_{y \in T_{\omega}^{-1}(x)} \hat{g}_{\omega}(y) f(y) \quad f \in \mathrm{BV}\left(X_{\omega}\right), x \in X_{\sigma(\omega)} .
$$

As an immediate consequence of (8.24) and (8.47), we get that

$$
\hat{\mathcal{L}}_{\omega} \mathbb{1}_{\omega}=\mathbb{1}_{\sigma(\omega)} \text {. }
$$

Furthermore, the operator $\hat{\mathcal{L}}_{\omega}$ satisfies the identity

$$
\hat{\mathcal{L}}_{\omega}\left(h \cdot\left(f \circ T_{\omega}^{n}\right)\right)=f \cdot \hat{\mathcal{L}}_{\omega}^{n} h
$$

for all $h \in \mathrm{BV}\left(X_{\omega}\right), f \in \mathrm{BV}\left(X_{\sigma^{n}(\omega)}\right)$, and all $n \in \mathbb{N}$. We end this section with the following proposition which shows that the family $\left(\mu_{\omega}\right)_{\omega \in \Omega}$ of measures is $T$-invariant.

Proposition 8.11. The family $\left(\mu_{\omega}\right)_{\omega \in \Omega}$ defined by (8.46) is T-invariant in the sense that

$$
\int_{X_{\omega}} f \circ T_{\omega} d \mu_{\omega}=\int_{X_{\sigma(\omega)}} f d \mu_{\sigma(\omega)}
$$

for $f \in L_{\mu_{\sigma(\omega)}}^{1}(I)=L_{\nu_{\sigma(\omega)}}^{1}(I)$.

Proof. In light of (3.12), we note that for $f \in L_{\mu_{\sigma(\omega)}}^{1}(I)$ we have

$$
\int_{X_{\sigma(\omega)}} \hat{\mathcal{L}}_{\omega} f d \mu_{\sigma(\omega)}=\int_{X_{\sigma(\omega)}} \tilde{\mathcal{L}}_{\omega}\left(f q_{\omega}\right) d \nu_{\sigma(\omega)}=\int_{X_{\omega}} f q_{\omega} d \nu_{\omega}=\int_{X_{\omega}} f d \mu_{\omega} .
$$

Now, if $h \cdot\left(f \circ T_{\omega}\right) \in L_{\mu_{\omega}}^{1}(I),(8.54)$ and (8.52) give that

$$
\int_{X_{\omega}} h \cdot\left(f \circ T_{\omega}\right) d \mu_{\omega}=\int_{X_{\sigma(\omega)}} \hat{\mathcal{L}}_{\omega}\left(h \cdot\left(f \circ T_{\omega}\right)\right) d \mu_{\sigma(\omega)}=\int_{X_{\sigma(\omega)}} f \cdot \hat{\mathcal{L}}_{\omega} h d \mu_{\sigma(\omega)} .
$$

In view of (8.51), taking $h=\mathbb{1}$ finishes the proof.

\section{RANDOM MEASURES}

In this section we show that the families of measures $\left(\nu_{\omega}\right)_{\omega, \in \Omega}$ and $\left(\mu_{\omega}\right)_{\omega, \in \Omega}$ are in fact random probability measures as defined in Definition 2.5 as well as show that the invariant density $q$ is unique. First we establish the existence of a measurable choice of an increasing sequence $\left(n_{k}(\omega)\right)_{k \geq 1}$ of positive integers along which we obtain the exponential convergence of $\hat{\mathcal{L}}_{\omega}^{n_{k}} f_{\omega}$.

Lemma 9.1. For $m$-a.e. $\omega \in \Omega$ there exists a measurable choice of an increasing sequence $\left(n_{k}(\omega)\right)_{k \geq 1}$ of positive integers such that if $f \in \prod_{\omega \in \Omega} \mathrm{BV}(I)$ with (D1) and (D2) holding for $\left(f, \omega, n_{k}(\omega)\right)$ for each $k \in \mathbb{N}$ and $\omega \in \Omega$, then there exists $\hat{A}_{f}: \Omega \rightarrow(0, \infty)$ such that

$$
\left\|\hat{\mathcal{L}}_{\omega}^{n_{k}} f_{\omega}-\mu_{\omega}\left(f_{\omega}\right) \mathbb{1}_{\sigma^{n_{k}(\omega)}}\right\|_{\infty} \leq \hat{A}_{f}(\omega)\left\|f_{\omega}\right\|_{\mathrm{BV}} \kappa^{n_{k}}
$$

Proof. Suppose first that $f \in \mathcal{D}^{+}$. In light of Lemma 8.8 item (a), we consider Corollary 8.9 (with $p=0$ ) for the function $\bar{f}=\left(f_{\omega} / \nu_{\omega}\left(f_{\omega}\right)\right)_{\omega \in \Omega} \in \mathcal{D}^{+}$, and thus, for each $n \geq N_{3}(\omega)$, we have that

$$
\left\|\tilde{\mathcal{L}}_{\omega}^{n}\left(\frac{f_{\omega}}{\nu_{\omega}\left(f_{\omega}\right)}\right)-q_{\sigma^{n}(\omega)}\right\|_{\infty} \leq A_{\bar{f}}(\omega)\left\|\frac{f_{\omega}}{\nu_{\omega}\left(f_{\omega}\right)}\right\|_{\mathrm{BV}} \kappa^{n}
$$


Multiplying both sides of $(9.1)$ by $\nu_{\omega}\left(f_{\omega}\right)$ yields

$$
\left\|\tilde{\mathcal{L}}_{\omega}^{n}\left(f_{\omega}\right)-\nu_{\omega}\left(f_{\omega}\right) q_{\sigma^{n}(\omega)}\right\|_{\infty} \leq A_{\bar{f}}(\omega)\left\|f_{\omega}\right\|_{\mathrm{BV}} \kappa^{n} .
$$

Now let $u(\omega)$ be the measurable lower bound for $q_{\omega}$ coming from Proposition 8.10, and choose $u_{*}>0$ sufficiently small such that

$$
m\left(\Omega_{*}:=\left\{\omega \in \Omega: u_{*} \leq u(\omega)\right\}\right)>0 .
$$

The ergodic theorem then gives that for $m$-a.e. $\omega \in \Omega$ there exists a measurable choice of an increasing sequence of positive integers $\left(n_{k}\right)_{k \geq 1}=\left(n_{k}(\omega)\right)_{k \geq 1}$ such that $\sigma^{n_{k}}(\omega) \in \Omega_{*}$ for each $k \geq 1$. It follows from Proposition 8.10 that

$$
0<u_{*} \leq u\left(\sigma^{n_{k}}(\omega)\right) \leq q_{\sigma^{n}(\omega)}
$$

for each $k \geq 1$. Then in light of Lemma 8.5 and (9.3), Lemma 8.8 item (b) implies that, for $m$-a.e. $\omega \in \Omega,(\mathcal{D} 1)$ and $(\mathcal{D} 2)$ hold for $\left(f q, \omega, n_{k}\right)$ for each $k \geq 1$. Thus, applying (9.2), with $n=n_{k}$ and the function $f_{\omega} q_{\omega}$ in place of $f_{\omega}$, gives

$$
\left\|\tilde{\mathcal{L}}_{\omega}^{n_{k}}\left(f_{\omega} q_{\omega}\right)-\mu_{\omega}\left(f_{\omega}\right) q_{\sigma^{n_{k}(\omega)}}\right\|_{\infty} \leq A_{\overline{f q}}(\omega)\left\|f_{\omega}\right\|_{\mathrm{BV}}\left\|q_{\omega}\right\|_{\mathrm{BV}} \kappa^{n_{k}}
$$

It then follows from (9.4) and (9.3) that we have

$$
\begin{aligned}
\left\|\hat{\mathcal{L}}_{\omega}^{n_{k}} f_{\omega}-\mu_{\omega}\left(f_{\omega}\right) \mathbb{1}_{\sigma^{n_{k}}(\omega)}\right\|_{\infty} & \leq \frac{1}{\inf q_{\sigma^{n_{k}}(\omega)}} \cdot\left\|\tilde{\mathcal{L}}_{\omega}^{n_{k}}\left(f_{\omega} q_{\omega}\right)-\mu_{\omega}\left(f_{\omega}\right) q_{\sigma^{n_{k}}(\omega)}\right\|_{\infty} \\
& \leq A_{\overline{f q}}(\omega) u_{*}^{-1}\left\|f_{\omega}\right\|_{\mathrm{BV}}\left\|q_{\omega}\right\|_{\mathrm{BV}} \kappa^{n_{k}} .
\end{aligned}
$$

Now, for a function $f \in \mathcal{D}$, we write $f=f^{+}-f^{-}$, with $f^{+}, f^{-} \geq 0$, and $f_{\omega}=f_{\omega}^{+}-f_{\omega}^{-}$for each $\omega \in \Omega$. Rerunning the previous argument with $f=f^{+}-f^{-}$to (9.5) and applying the triangle inequality we get

$$
\left\|\hat{\mathcal{L}}_{\omega}^{n_{k}} f_{\omega}-\mu_{\omega}\left(f_{\omega}\right) \mathbb{1}_{\sigma^{n_{k}(\omega)}}\right\|_{\infty} \leq \hat{A}_{f}(\omega)\left\|f_{\omega}\right\|_{\mathrm{BV}} \kappa^{n_{k}}
$$

for each $k \in \mathbb{N}$, where

$$
\hat{A}_{f}(\omega):=\left(A_{\overline{f^{+}} q}(\omega)+A_{\overline{f^{-} q}}(\omega)\right)\left\|q_{\omega}\right\|_{\mathrm{BV}} u_{*}^{-1}
$$

In the following lemma we give a characterization of the measure $\nu_{\omega}$ as a limit of measurable functions along the measurable sequence $n_{k}$ produced in the previous lemma.

Lemma 9.2. For each $f \in \mathcal{D}$ and $m$-a.e. $\omega \in \Omega$ we have

$$
\nu_{\omega}\left(f_{\omega}\right)=\lim _{k \rightarrow \infty} \frac{\left\|\mathcal{L}_{\omega}^{n_{k}} f_{\omega}\right\|_{\infty}}{\left\|\mathcal{L}_{\omega}^{n_{k}} \mathbb{1}_{\omega}\right\|_{\infty}}
$$

where $\left(n_{k}(\omega)\right)_{k \geq 1}$ is the measurable sequence of positive integers coming from Lemma 9.1.

Proof. Let $\left(n_{k}\right)_{k \geq 1}=\left(n_{k}(\omega)\right)_{k \geq 1}$ denote the measurable sequence of positive integers coming from Lemma 9.1. In light of Lemma 8.5, Lemma 8.8 item (c) implies that, for $m$-a.e. $\omega \in \Omega$, $(\mathcal{D} 1)$ holds for $\left(f / q, \omega, n_{k}\right)$ for each $k \geq 1$. Furthermore, the uniform lower bound for $q_{\omega}$ given in (9.3) together with Lemma 8.8 item (c) implies that, for $m$-a.e. $\omega \in \Omega$, (D2) holds for $\left(f / q, \omega, n_{k}\right)$ for each $k \geq 1$. Thus Lemma 9.1 gives that

$$
\|\| \hat{\mathcal{L}}_{\omega}^{n_{k}}\left(\frac{f_{\omega}}{q_{\omega}}\right)\left\|_{\infty}-\nu_{\omega}\left(f_{\omega}\right) \mid \leq \hat{A}_{\frac{f}{q}}(\omega)\right\| \frac{f_{\omega}}{q_{\omega}} \|_{\mathrm{BV}} \kappa^{n_{k}}
$$


for $m$-a.e. $\omega \in \Omega$ and each $k \in \mathbb{N}$. Hence, taking limits, we get

$$
\lim _{k \rightarrow \infty} \frac{\left\|\mathcal{L}_{\omega}^{n_{k}} f_{\omega}\right\|_{\infty}}{\left\|\mathcal{L}_{\omega}^{n_{k}} \mathbb{1}_{\omega}\right\|_{\infty}}=\lim _{k \rightarrow \infty} \frac{\left\|\hat{\mathcal{L}}_{\omega}^{n_{k}}\left(\frac{f_{\omega}}{q_{\omega}}\right)\right\|_{\infty}}{\left\|\hat{\mathcal{L}}_{\omega}^{n_{k}}\left(\frac{\mathbb{1}_{\omega}}{q_{\omega}}\right)\right\|_{\infty}}=\frac{\nu_{\omega}\left(f_{\omega}\right)}{\nu_{\omega}\left(\mathbb{1}_{\omega}\right)}=\nu_{\omega}\left(f_{\omega}\right) .
$$

We now arrive at the main result of this section.

Proposition 9.3. The map $\omega \mapsto \lambda_{\omega}$ is measurable with $\log \lambda_{\omega} \in L_{m}^{1}(\Omega)$ and the families $\left(\nu_{\omega}\right)_{\omega \in \Omega},\left(\mu_{\omega}\right)_{\omega \in \Omega}$ are random probability measures giving rise to $\nu, \mu \in \mathcal{P}_{m}(\Omega \times I)$ defined by

$$
\nu(f)=\int_{\Omega} \int_{I} f_{\omega} d \nu_{\omega} d m(\omega) \quad \text { and } \quad \mu(f)=\int_{\Omega} \int_{I} f_{\omega} d \mu_{\omega} d m(\omega)
$$

for $f \in L_{\nu}^{1}(\Omega \times I)$. Furthermore, the maps $\omega \mapsto\left\|q_{\omega}\right\|_{\infty}$ and $\omega \mapsto \inf q_{\omega}$ are measurable.

Proof. We prove the proposition in four steps:

(i) We first show that $\left(\nu_{\omega}\right)_{\omega \in \Omega}$ is a random probability measure as in Definition 2.5. Indeed, for every interval $J \subset I$, the function $\omega \mapsto \nu_{\omega}(J)$ is measurable, as it is the limit of measurable functions by $(9.7)$ applied to $f_{\omega}=\mathbb{1}_{J}$. Since $\mathscr{B}$ is generated by intervals, $\omega \mapsto \nu_{\omega}(B)$ is measurable for every $B \in \mathscr{B}$. Furthermore, $\nu_{\omega}$ is a Borel probability measure for $m$-a.e. $\omega \in \Omega$ from Proposition 3.1.

(ii) Given that $\lambda_{\omega}:=\nu_{\sigma(\omega)}\left(\mathcal{L}_{\omega} \mathbb{1}_{\omega}\right)$, (i) and Proposition 2.6 immediately imply that $\omega \mapsto \lambda_{\omega}$ is measurable. The log-integrability claim then follows from Lemma 2.13, and the fact that

$$
\inf \mathcal{L}_{\omega} \mathbb{1}_{\omega} \leq \lambda_{\omega} \leq\left\|\mathcal{L}_{\omega} \mathbb{1}_{\omega}\right\|_{\infty}
$$

(iii) The measurability of $\lambda_{\omega}$ together with (M2) and the fact that

$$
q_{\omega}=\lim _{n \rightarrow \infty}\left(\lambda_{\omega}^{n}\right)^{-1} \mathcal{L}_{\sigma^{-n}(\omega)}^{n} \mathbb{1}_{\sigma^{-n}(\omega)}
$$

(see (8.24)) yield the measurability conditions on $q$.

(iv) The Borel probability measures $\left(\mu_{\omega}\right)_{\omega \in \Omega}$ also define a random probability measure. Indeed, because intervals generate $\mathscr{B}$, it is enough to check that for every interval $J \subset I$, the function $\omega \mapsto \mu_{\omega}(J)$ is measurable. This follows from (iii) and the fact that $\mu_{\omega}(J)$ is the limit of measurable functions, coming from (9.7) applied to $f_{\omega}=\mathbb{1}_{J} q_{\omega}$.

To end this section we now prove the uniqueness of the invariant density $q$.

Proposition 9.4. The global invariant density $q \in \mathrm{BV}_{\Omega}(I)$ produced in Corollary 8.3 is the unique element of $L_{\nu}^{1}(\Omega \times I)$ (modulo $\left.\nu\right)$ such that

$$
\tilde{\mathcal{L}}_{\omega} q_{\omega}=q_{\sigma(\omega)} .
$$

Proof. Towards a contradiction, suppose that there exists $\psi \in L_{\nu}^{1}(\Omega \times I)$ with $\|\psi\|_{L_{\nu}^{1}}=1$ such that

$$
\tilde{\mathcal{L}}_{\omega} \psi_{\omega}=\psi_{\sigma(\omega)} .
$$


By ergodicity we must have that $\left\|\psi_{\omega}\right\|_{L_{\nu_{\omega}}^{1}}=1$ for $m$-a.e. $\omega \in \Omega$. Since $\operatorname{BV}\left(X_{\omega}\right)$ is dense in $L_{\nu_{\omega}}^{1}\left(X_{\omega}\right)$ for each $\omega \in \Omega$, and in particular the set $\left\{f \in \mathrm{BV}(I):\|f\|_{L_{\nu_{\omega}}^{1}}=1\right\}$ is dense in the set $\left\{f \in L_{\nu_{\omega}}^{1}(I):\|f\|_{L_{\nu_{\omega}}^{1}}=1\right\}$, for each $\delta>0$ we can find $f \in \mathrm{BV}_{\Omega}(I)$ with $\left\|f_{\omega}\right\|_{L_{\nu_{\omega}}^{1}}=1$ such that we have

$$
\left\|\psi_{\omega}-f_{\omega}\right\|_{L_{\nu_{\omega}}^{1}} \leq \delta
$$

for $m$-a.e. $\omega \in \Omega$. Now let $V_{\psi}>0$ be sufficiently large such that

$$
m\left(\Omega_{\psi}:=\left\{\omega \in \Omega: \operatorname{var}\left(f_{\omega}\right) \leq V_{\psi}\right\}\right)>0,
$$

and for each $\omega \in \Omega$ we define the function $\tilde{\psi} \in \mathrm{BV}_{\Omega}(I)$ by

$$
\tilde{\psi}_{\omega}:= \begin{cases}f_{\omega} & \text { if } \omega \in \Omega_{\psi} \\ \mathbb{1}_{\omega} & \text { if } \omega \notin \Omega_{\psi} .\end{cases}
$$

Thus, using (9.12) and (9.13) we have that

(9.14) $\operatorname{var}\left(\tilde{\psi}_{\omega}\right) \leq V_{\psi}<\infty$,

$$
\left\|\tilde{\psi}_{\omega}\right\|_{L_{\nu_{\omega}}^{1}}=1
$$

for each $\omega \in \Omega$ and using (9.11) we see that

$$
\left\|\psi_{\omega}-\tilde{\psi}_{\omega}\right\|_{L_{\nu_{\omega}}^{1}} \leq \delta
$$

for each $\omega \in \Omega_{\psi}$. Now in light of (9.14) and (9.15) we see that $\tilde{\psi} \in \mathcal{D}$, and thus we apply Corollary 8.9 , and more specifically equation (8.40), to see that

$$
\left\|q_{\omega}-\tilde{\mathcal{L}}_{\sigma^{-n}(\omega)}^{n} \tilde{\psi}_{\sigma^{-n}(\omega)}\right\|_{\infty} \leq\left\|q_{\omega}\right\|_{\infty} \tilde{\kappa}^{n}
$$

for all $n \in \mathbb{N}$ sufficiently large, where $\tilde{\kappa} \in(0,1)$. Next we note that using (3.12) and (9.10) gives that

$$
\begin{aligned}
\left\|\psi_{\omega}-\tilde{\mathcal{L}}_{\sigma^{-n}(\omega)}^{n} \tilde{\psi}_{\sigma^{-n}(\omega)}\right\|_{L_{\nu_{\omega}}^{1}} & =\left\|\tilde{\mathcal{L}}_{\sigma^{-n}(\omega)}^{n} \psi_{\sigma^{-n}(\omega)}-\tilde{\mathcal{L}}_{\sigma^{-n}(\omega)}^{n} \tilde{\psi}_{\sigma^{-n}(\omega)}\right\|_{L_{\nu_{\omega}}^{1}} \\
& \leq \int_{I} \tilde{\mathcal{L}}_{\sigma^{-n}(\omega)}^{n}\left(\left|\psi_{\sigma^{-n}(\omega)}-\tilde{\psi}_{\sigma^{-n}(\omega)}\right|\right) d \nu_{\omega} \\
& =\left\|\psi_{\sigma^{-n}(\omega)}-\tilde{\psi}_{\sigma^{-n}(\omega)}\right\|_{L_{\nu_{\sigma^{-n}}(\omega)}^{1}}
\end{aligned}
$$

for each $n \in \mathbb{N}$. It then follows from (9.12), the ergodic theorem, (9.16), and (9.18) that for $m$-a.e. $\omega \in \Omega$ there are infinitely many $n \in \mathbb{N}$ such that

$$
\left\|\psi_{\omega}-\tilde{\mathcal{L}}_{\sigma^{-n}(\omega)}^{n} \tilde{\psi}_{\sigma^{-n}(\omega)}\right\|_{L_{\nu_{\omega}}^{1}} \leq\left\|\psi_{\sigma^{-n}(\omega)}-\tilde{\psi}_{\sigma^{-n}(\omega)}\right\|_{L_{\nu_{\sigma^{-n}}(\omega)}^{1}} \leq \delta .
$$

Thus, in light of (9.17) and (9.19), for $m$-a.e. $\omega \in \Omega$ we have

$$
\left\|\psi_{\omega}-q_{\omega}\right\|_{L_{\nu_{\omega}}^{1}} \leq\left\|q_{\omega}-\tilde{\mathcal{L}}_{\sigma^{-n}(\omega)} \tilde{\psi}_{\sigma^{-n}(\omega)}\right\|_{L_{\nu_{\omega}}^{1}}+\left\|\psi_{\omega}-\tilde{\mathcal{L}}_{\sigma^{-n}(\omega)}^{n} \tilde{\psi}_{\sigma^{-n}(\omega)}\right\|_{L_{\nu_{\omega}}^{1}} \leq 2 \delta
$$

for infinitely many $n \in \mathbb{N}$ sufficiently large. As this holds for each $\delta>0$, we must in fact have that $\psi_{\omega}=q_{\omega}$ modulo $\nu_{\omega}$ for $m$-a.e. $\omega \in \Omega$, which implies that $\psi=q$ modulo $\nu$ as desired. 


\section{Expected Pressure}

In this section we exploit the measurability produced in Proposition 9.3. We begin by defining the expected pressure, as in [48], and then prove an alternate characterization of the expected pressure in terms of limits. An important consequence of the proof of this characterization is that we obtain the temperedness of the quantities inf $q_{\omega}$ and $\left\|q_{\omega}\right\|_{\infty}$, which allows us to prove improved versions of Lemmas 9.1 and 9.2, thus completing the proof of Proof of Theorem 2.20.

Towards that end, given that $\log \lambda_{\omega} \in L_{m}^{1}(\Omega)$ by Proposition 9.3 , we define the expected pressure by

$$
\mathcal{E} P(\varphi):=\int_{\Omega} \log \lambda_{\omega} d m(\omega)=\lim _{n \rightarrow \infty} \frac{1}{n} \log \lambda_{\omega}^{n}=\lim _{n \rightarrow \infty} \frac{1}{n} \log \lambda_{\sigma^{-n}(\omega)}^{n},
$$

where the second and third equalities hold $m$-a.e. and follow from Birkhoff's Ergodic Theorem. The following lemma provides alternate characterizations of the expected pressure.

Lemma 10.1. For $m$-a.e. $\omega \in \Omega$ we have that

$$
\lim _{n \rightarrow \infty}\left\|\frac{1}{n} \log \mathcal{L}_{\sigma^{-n}(\omega)}^{n} \mathbb{1}_{\sigma^{-n}(\omega)}-\frac{1}{n} \log \lambda_{\sigma^{-n}(\omega)}^{n}\right\|_{\infty}=0
$$

and

$$
\lim _{n \rightarrow \infty}\left\|\frac{1}{n} \log \mathcal{L}_{\omega}^{n} \mathbb{1}_{\omega}-\frac{1}{n} \log \lambda_{\omega}^{n}\right\|_{\infty}=0
$$

Furthermore, we have that

$$
\lim _{n \rightarrow \infty} \frac{1}{n} \log \inf q_{\sigma^{n}(\omega)}=\lim _{n \rightarrow \infty} \frac{1}{n} \log \left\|q_{\sigma^{n}(\omega)}\right\|_{\infty}=0 .
$$

Proof. Towards proving (10.2), Corollary 8.9 (with $p=-n$ and $f_{\sigma^{-n}(\omega)}=\mathbb{1}_{\sigma^{-n}(\omega)}$ ) allows us to write

$$
\inf q_{\omega}-A_{\mathbb{1}}(\omega) \kappa^{n} \leq \frac{\mathcal{L}_{\sigma^{-n}(\omega)}^{n} \mathbb{1}_{\sigma^{-n}(\omega)}}{\lambda_{\sigma^{-n}(\omega)}^{n}} \leq\left\|q_{\omega}\right\|_{\infty}+A_{\mathbb{1}}(\omega) \kappa^{n}
$$

for each $n \in \mathbb{N}$. Thus, for $n$ sufficiently large, we have

$$
\frac{1}{n} \log \left(\inf q_{\omega}-A_{\mathbb{1}}(\omega) \kappa^{n}\right) \leq \frac{1}{n} \log \frac{\mathcal{L}_{\sigma^{-n}(\omega)}^{n} \mathbb{1}_{\sigma^{-n}(\omega)}}{\lambda_{\sigma^{-n}(\omega)}^{n}} \leq \frac{1}{n} \log \left(\left\|q_{\omega}\right\|_{\infty}+A_{\mathbb{1}}(\omega) \kappa^{n}\right) .
$$

Since Proposition 8.10 implies that $\log \inf q_{\omega}>-\infty$ and $\log \left\|q_{\omega}\right\|_{\infty}<\infty$, letting $n \rightarrow \infty$ finishes the first claim.

Before continuing on to prove the second claim, we first note that Corollary 8.9 implies that

$$
\inf q_{\sigma^{n}(\omega)} \geq \inf \tilde{\mathcal{L}}_{\omega}^{n} \mathbb{1}_{\omega}-A_{\mathbb{1}}(\omega) \kappa^{n},
$$

for all $n \in \mathbb{N}$ sufficiently large, and thus,

$$
\frac{1}{n} \log \inf q_{\sigma^{n}(\omega)} \geq \frac{1}{n} \log \left(\inf \tilde{\mathcal{L}}_{\omega}^{n} \mathbb{1}_{\omega}-A_{\mathbb{1}}(\omega) \kappa^{n}\right) .
$$

It follows from (10.2) that inf $\tilde{\mathcal{L}}_{\omega} \mathbb{1}_{\omega}$ is tempered, and thus for each $0<\delta<\kappa$ there exists a $m$-a.e. finite function $K_{\delta}: \Omega \rightarrow(0, \infty)$ such that

$$
\inf \tilde{\mathcal{L}}_{\omega}^{n} \mathbb{1}_{\omega} \geq K_{\delta}(\omega) e^{-\delta n}>A_{\mathbb{1}}(\omega) \kappa^{n}
$$


for each $n \in \mathbb{N}$. Inserting (10.5) into (10.4) and taking a limit as $n \rightarrow \infty$ gives

$$
\begin{aligned}
\lim _{n \rightarrow \infty} \frac{1}{n} \log \inf q_{\sigma^{n}(\omega)} & \geq \lim _{n \rightarrow \infty} \frac{1}{n} \log \left(\inf \tilde{\mathcal{L}}_{\omega}^{n} \mathbb{1}_{\omega}-A_{\mathbb{1}}(\omega) \kappa^{n}\right) \\
& \geq \lim _{n \rightarrow \infty} \frac{1}{n} \log \left(K_{\delta}(\omega) e^{-\delta n}-A_{\mathbb{1}}(\omega) \kappa^{n}\right) \\
& =\lim _{n \rightarrow \infty} \frac{1}{n} \log \left(K_{\delta}(\omega) e^{-\delta n}\right)=-\delta .
\end{aligned}
$$

As this holds for every $\delta>0$ we must in fact have that

$$
\lim _{n \rightarrow \infty} \frac{1}{n} \log \inf q_{\sigma^{n}(\omega)}=\lim _{n \rightarrow \infty} \frac{1}{n} \log \left\|q_{\sigma^{n}(\omega)}\right\|_{\infty}=0,
$$

where we recall that the second equality follows from Lemma 8.5. Now to prove the second claim, we again note that using Corollary 8.9 gives that for each $n \in \mathbb{N}$ we have

$$
\inf q_{\sigma^{n}(\omega)}-A_{\mathbb{1}}(\omega) \kappa^{n} \leq \frac{\mathcal{L}_{\omega}^{n} \mathbb{1}_{\omega}}{\lambda_{\omega}^{n}} \leq\left\|q_{\sigma^{n}(\omega)}\right\|_{\infty}+A_{\mathbb{1}}(\omega) \kappa^{n},
$$

and hence, for $n$ sufficiently large, we have

$$
\frac{1}{n} \log \left(\inf q_{\sigma^{n}(\omega)}-A_{\mathbb{1}}(\omega) \kappa^{n}\right) \leq \frac{1}{n} \log \frac{\mathcal{L}_{\omega}^{n} \mathbb{1}_{\omega}}{\lambda_{\omega}^{n}} \leq \frac{1}{n} \log \left(\left\|q_{\sigma^{n}(\omega)}\right\|_{\infty}+A_{\mathbb{1}}(\omega) \kappa^{n}\right) .
$$

In view of Proposition 8.10 and (10.6), we see that letting $n \rightarrow \infty$ in (10.7) finishes the proof of the second claim and thus the lemma.

The following corollary ensures the measurability of the coefficients $A_{f}$ and $\hat{A}_{f}$ which appeared respectively in Corollary 8.9 and Lemma 9.1.

Corollary 10.2. For each $f \in \mathrm{BV}_{\Omega}^{l}(I)$ the maps $\omega \mapsto A_{f}(\omega)$ and $\omega \mapsto \hat{A}_{f}(\omega)$ are measurable and $f / \nu(f), f q, f / q \in \mathcal{D}$.

Proof. From Remark 8.7, we see that $\mathrm{BV}_{\Omega}^{l}(I) \subseteq \mathcal{D}$ and using (10.6), Proposition 8.10, and Lemma 8.8, we have that $f / \nu(f), f q, f / q \in \mathcal{D}$ for each $f \in \mathrm{BV}_{\Omega}^{l}(I)$.

Now to see the claim that the random coefficients $A_{f}$ and $\hat{A}_{f}$ are measurable we first note that the map $\omega \mapsto V_{f, \varepsilon}(\omega)$ from Definition 8.6, which depends on $\left\|f_{\omega}\right\|_{\mathrm{BV}}$ and $\left\|f_{\omega}\right\|_{L_{\nu^{\prime}}^{1}(I)}$, can be chosen measurably for each $f \in \mathrm{BV}_{\Omega}^{l}(I)$. Thus the map $\omega \mapsto V(\omega)$, defined in (8.39), must also be measurable. In light of Remark 8.2, we see that the map $\omega \mapsto N_{3}(\omega)$, defined by (8.13), is measurable, which then implies that the map $\omega \mapsto A_{f}(\omega)$ is measurable for each $f \in \mathrm{BV}_{\Omega}^{l}(I)$. Furthermore, since $N_{3}(\omega)$ depends on $\left\|f_{\omega}\right\|_{\mathrm{BV}}$ and $\left\|f_{\omega}\right\|_{L_{\nu_{\omega}}^{1}(I)}\left(\right.$ via $\left.V_{f, \varepsilon}(\omega)\right)$, so does $A_{f}(\omega)$. The measurability of $\hat{A}_{f}$ then follows from (9.6).

Now in light of the proof of Lemma 10.1, in particular (10.6), we can give improved versions of Lemmas 9.1 and 9.2 which no longer depend upon a particular sequence of integers $n_{k}$.

Theorem 10.3. For each $f \in \mathrm{BV}_{\Omega}^{l}(I)$ and each $\varkappa \in(\kappa, 1)$ there exist measurable functions $B_{f}, C_{f}: \Omega \rightarrow(0, \infty)$ such that for $m$-a.e. $\omega \in \Omega$, all $n \in \mathbb{N}$, and all $|p| \leq n$ we have

$$
\left\|\tilde{\mathcal{L}}_{\sigma^{p}(\omega)}^{n} f_{\sigma^{p}(\omega)}-\nu_{\sigma^{p}(\omega)}\left(f_{\sigma^{p}(\omega)}\right) q_{\sigma^{p+n}(\omega)}\right\|_{\infty} \leq B_{f}(\omega)\left\|f_{\sigma^{p}(\omega)}\right\|_{\mathrm{BV}} \kappa^{n}
$$

and

$$
\left\|\hat{\mathcal{L}}_{\sigma^{p}(\omega)}^{n} f_{\sigma^{p}(\omega)}-\mu_{\sigma^{p}(\omega)}\left(f_{\sigma^{p}(\omega)}\right) \mathbb{1}_{\sigma^{p+n}(\omega)}\right\|_{\infty} \leq C_{f}(\omega)\left\|f_{\sigma^{p}(\omega)}\right\|_{\mathrm{BV}} \varkappa^{n}
$$


where $\kappa<1$ is as in Corollary 8.9 .

Proof. Suppose first that $f \in \mathrm{BV}_{\Omega}^{l}(I)$ with $f \geq 0$. Since Lemma 8.8 item (a) ensures that $\bar{f}=\left(f_{\omega} / \nu_{\omega}\left(f_{\omega}\right)\right)_{\omega \in \Omega} \in \mathcal{D}^{+}$, we apply Corollary 8.9 to obtain

$$
\left\|\tilde{\mathcal{L}}_{\sigma^{p}(\omega)}^{n}\left(\frac{f_{\sigma^{p}(\omega)}}{\nu_{\sigma^{p}(\omega)}\left(f_{\left.\sigma^{p}(\omega)\right)}\right)}\right)-q_{\sigma^{n+p}(\omega)}\right\|_{\infty} \leq A_{\bar{f}}(\omega)\left\|\frac{f_{\sigma^{p}(\omega)}}{\nu_{\sigma^{p}(\omega)}\left(f_{\sigma^{p}(\omega)}\right)}\right\|_{\mathrm{BV}} \kappa^{n}
$$

for each $n \in \mathbb{N}$, where $A_{\bar{f}}(\omega)$ is as in Corollary 8.9. Multiplying both sides of (10.8) by $\nu_{\sigma^{p}(\omega)}\left(f_{\sigma^{p}(\omega)}\right)$ yields

$$
\left\|\tilde{\mathcal{L}}_{\sigma^{p}(\omega)}^{n}\left(f_{\sigma^{p}(\omega)}\right)-\nu_{\sigma^{p}(\omega)}\left(f_{\sigma^{p}(\omega)}\right) q_{\sigma^{n+p}(\omega)}\right\|_{\infty} \leq A_{\bar{f}}(\omega)\left\|f_{\sigma^{p}(\omega)}\right\|_{\mathrm{BV}} \kappa^{n} .
$$

In view of Corollary 10.2, applying (10.9) with the function $f q \in \mathcal{D}^{+}$gives

$$
\left\|\tilde{\mathcal{L}}_{\sigma^{p}(\omega)}^{n}\left(f_{\sigma^{p}(\omega)} q_{\sigma^{p}(\omega)}\right)-\mu_{\sigma^{p}(\omega)}\left(f_{\sigma^{p}(\omega)}\right) q_{\sigma^{n+p}(\omega)}\right\|_{\infty} \leq A_{\overline{f q}}(\omega)\left\|f_{\sigma^{p}(\omega)}\right\|_{\mathrm{BV}}\left\|q_{\sigma^{p}(\omega)}\right\|_{\mathrm{BV}} \kappa^{n}
$$

for each $n \in \mathbb{N}$. It follows from (10.10) that

$$
\begin{aligned}
& \left\|\hat{\mathcal{L}}_{\sigma^{p}(\omega)}^{n} f_{\sigma^{p}(\omega)}-\mu_{\sigma^{p}(\omega)}\left(f_{\sigma^{p}(\omega)}\right) \mathbb{1}_{\sigma^{p+n}(\omega)}\right\|_{\infty} \\
& \quad \leq \frac{1}{\inf q_{\sigma^{p+n}(\omega)}} \cdot\left\|\tilde{\mathcal{L}}_{\sigma^{p}(\omega)}^{n}\left(f_{\sigma^{p}(\omega)} q_{\sigma^{p}(\omega)}\right)-\mu_{\sigma^{p}(\omega)}\left(f_{\sigma^{p}(\omega)}\right) q_{\sigma^{p+n}(\omega)}\right\|_{\infty} \\
& \quad \leq A \overline{f q}(\omega)\left\|f_{\sigma^{p}(\omega)}\right\|_{\mathrm{BV}}\left\|q_{\sigma^{p}(\omega)}\right\|_{\mathrm{BV}} \frac{1}{\inf q_{\sigma^{p+n}(\omega)}} \kappa^{n}
\end{aligned}
$$

for each $n \in \mathbb{N}$. Now in light of (10.6), we have that

$$
\lim _{n \rightarrow \infty} \frac{1}{n} \log \frac{\left\|q_{\sigma^{n}(\omega)}\right\|_{\mathrm{BV}}}{\inf q_{\sigma^{n}(\omega)}}=0,
$$

and thus, for each $\delta>0$, there exists a measurable constant $D(\omega, \delta)>0$ such that

$$
\frac{\left\|q_{\sigma^{n}(\omega)}\right\|_{\mathrm{BV}}}{\inf q_{\sigma^{n}(\omega)}} \leq D(\omega, \delta) e^{\delta n}
$$

for each $n \in \mathbb{N}$. Inserting (10.12) into (10.11), for $\delta>0$ sufficiently small and each $n \in \mathbb{N}$ we have

$$
\left\|\hat{\mathcal{L}}_{\sigma^{p}(\omega)}^{n} f_{\sigma^{p}(\omega)}-\mu_{\sigma^{p}(\omega)}\left(f_{\sigma^{p}(\omega)}\right) \mathbb{1}_{\sigma^{p+n}(\omega)}\right\|_{\infty} \leq A_{\overline{f q}}(\omega) D(\omega, \delta)\left\|f_{\sigma^{p}(\omega)}\right\|_{\mathrm{BV}} \varkappa^{n}
$$

where $0<\kappa<\varkappa:=\kappa e^{\delta}<1$.

Now, we write a function $f \in \mathrm{BV}_{\Omega}^{l}(I)$ as $f=f^{+}-f^{-}$with $f^{+}, f^{-} \geq 0$, and apply the triangle inequality with (10.9) to obtain

$$
\left\|\tilde{\mathcal{L}}_{\sigma^{p}(\omega)}^{n} f_{\sigma^{p}(\omega)}-\nu_{\sigma^{p}(\omega)}\left(f_{\sigma^{p}(\omega)}\right) q_{\sigma^{p+n}(\omega)}\right\|_{\infty} \leq B_{f}(\omega)\left\|f_{\sigma^{p}(\omega)}\right\|_{\mathrm{BV}} \kappa^{n}
$$

where

$$
B_{f}(\omega):=\left(A_{\overline{f^{+}}}(\omega)+A_{\overline{f^{-}}}(\omega)\right) .
$$

Similarly, using the triangle inequality with (10.13) yields

$$
\left\|\hat{\mathcal{L}}_{\sigma^{p}(\omega)}^{n} f_{\sigma^{p}(\omega)}-\mu_{\sigma^{p}(\omega)}\left(f_{\sigma^{p}(\omega)}\right) \mathbb{1}_{\sigma^{p+n}(\omega)}\right\|_{\infty} \leq C_{f}(\omega)\left\|f_{\sigma^{p}(\omega)}\right\|_{\mathrm{BV}} \varkappa^{n},
$$


where

$$
C_{f}(\omega):=D(\omega, \delta)\left(A \overline{f^{+} q}(\omega)+A \overline{f^{-} q}(\omega)\right) .
$$

To finish the proof we note that claim that $B_{f}$ and $C_{f}$ are measurable for $f \in \mathrm{BV}_{\Omega}^{l}(I)$ follows from Corollary 10.2 and equations (10.14) and (10.15).

The following Proposition is an improvement on Lemma 9.2 in that we prove a general limit for the measures $\nu_{\omega}$, rather than a limit along a subsequence. Furthermore, we now prove that the random measure $\nu$ is unique.

Proposition 10.4. The family $\left(\nu_{\omega}\right)_{\omega \in \Omega}$ of probability measures is uniquely determined by (3.1). In particular, we have that for each $f \in \mathrm{BV}_{\Omega}^{l}(I)$

$$
\nu_{\omega}\left(f_{\omega}\right)=\lim _{n \rightarrow \infty} \frac{\left\|\mathcal{L}_{\omega}^{n} f_{\omega}\right\|_{\infty}}{\left\|\mathcal{L}_{\omega}^{n} \mathbb{1}_{\omega}\right\|_{\infty}} .
$$

Furthermore, we have that the family $\left(\mu_{\omega}\right)_{\omega \in \Omega}$ of $T$-invariant measures is also unique.

Proof. Suppose $\left(\nu_{\omega}^{\prime}\right)_{\omega \in \Omega}$ is another family of probability measures which satisfies (3.1), i.e.

$$
\nu_{\sigma(\omega)}^{\prime}\left(\mathcal{L}_{\omega} f_{\omega}\right)=\lambda_{\omega}^{\prime} \nu_{\omega}^{\prime}\left(f_{\omega}\right), \quad \text { with } \lambda_{\omega}^{\prime}=\nu_{\sigma(\omega)}^{\prime}\left(\mathcal{L}_{\omega} \mathbb{1}_{\omega}\right), \quad f \in \mathrm{BV}_{\Omega}^{l}(I) .
$$

Further define $\mu_{\omega}^{\prime}=q_{\omega} \nu_{\omega}^{\prime}$ for each $\omega \in \Omega$. For each $h \in \mathrm{BV}_{\Omega}^{l}(I)$ Theorem 10.3 (taken with $p=0$ ) applied to the measure $\mu_{\omega}^{\prime}$ gives that

$$
\left|\left\|\hat{\mathcal{L}}_{\omega}^{n} h_{\omega}\right\|_{\infty}-\mu_{\omega}^{\prime}\left(h_{\omega}\right)\right| \leq C_{h}(\omega)\left\|h_{\omega}\right\|_{\mathrm{BV}} \varkappa^{n}
$$

for each $n \in \mathbb{N}$. Inserting the function $f / q \in \mathcal{D}$, for $h \in \mathrm{BV}_{\Omega}^{l}(I)$, into the previous estimate gives

$$
\left\|\hat{\mathcal{L}}_{\omega}^{n}\left(\frac{f_{\omega}}{q_{\omega}}\right)\right\|_{\infty}-\nu_{\omega}^{\prime}\left(f_{\omega}\right) \mid \leq C_{\frac{f}{q}}(\omega)\left\|\frac{f_{\omega}}{q_{\omega}}\right\|_{\mathrm{BV}} \varkappa^{n}
$$

for each $n \in \mathbb{N}$. Thus we have

$$
\lim _{n \rightarrow \infty} \frac{\left\|\mathcal{L}_{\omega}^{n} f_{\omega}\right\|_{\infty}}{\left\|\mathcal{L}_{\omega}^{n} \mathbb{1}_{\omega}\right\|_{\infty}}=\lim _{n \rightarrow \infty} \frac{\left\|\hat{\mathcal{L}}_{\omega}^{n}\left(\frac{f_{\omega}}{q_{\omega}}\right)\right\|_{\infty}}{\left\|\hat{\mathcal{L}}_{\omega}^{n}\left(\frac{\mathbb{1}_{\omega}}{q_{\omega}}\right)\right\|_{\infty}}=\frac{\nu_{\omega}^{\prime}\left(f_{\omega}\right)}{\nu_{\omega}^{\prime}\left(\mathbb{1}_{\omega}\right)}=\nu_{\omega}^{\prime}\left(f_{\omega}\right) .
$$

As the limit on the left-hand side has no dependence on the family $\nu_{\omega}^{\prime}$, the uniqueness of the limit, and of the density $q_{\omega}$, implies the uniqueness of the families $\left(\nu_{\omega}\right)_{\omega \in \Omega}$ and $\left(\mu_{\omega}\right)_{\omega \in \Omega}$.

We are now ready to prove Theorem 2.20.

Proof of Theorem 2.20. Theorem 2.20 follows from Theorem 10.3 with $p=-n$ and $p=$ 0.

\section{Decay of Correlations}

In this short section we prove Theorem 2.21, i.e. the forward and backward exponential decay of correlations. As a consequence of this, we obtain that the invariant measure $\mu$ is ergodic, thus completing the proof of Theorem 2.19. 
Theorem 11.1. For $m$-a.e. every $\omega \in \Omega$, every $n \in \mathbb{N}$, every $|p| \leq n$, every $f \in L_{\mu}^{1}(\Omega \times I)$, and every $h \in \mathrm{BV}_{\Omega}^{l}(I)$ we have

$$
\left|\mu_{\tau}\left(\left(f_{\sigma^{n}(\tau)} \circ T_{\tau}^{n}\right) h_{\tau}\right)-\mu_{\sigma^{n}(\tau)}\left(f_{\sigma^{n}(\tau)}\right) \mu_{\tau}\left(h_{\tau}\right)\right| \leq C_{h}(\omega)\left\|f_{\sigma^{n}(\tau)}\right\|_{L_{\mu_{\sigma^{n}(\tau)}^{1}}}\left\|h_{\tau}\right\|_{\mathrm{BV}} \varkappa^{n},
$$

where $\tau=\sigma^{p}(\omega)$, and where $C_{h}(\omega)$ and $\varkappa$ are as in Theorem 10.3.

Proof. First let $\hat{h}_{\omega}=h_{\omega}-\mu_{\omega}\left(h_{\omega}\right)$ for each $\omega \in \Omega$. Now, we note that

$$
\begin{aligned}
\mu_{\tau}\left(\left(f_{\sigma^{n}(\tau)} \circ T_{\tau}^{n}\right) h_{\tau}\right)-\mu_{\sigma^{n}(\tau)}\left(f_{\sigma^{n}(\tau)}\right) \mu_{\tau}\left(h_{\tau}\right) \\
=\mu_{\sigma^{n}(\tau)}\left(f_{\sigma^{n}(\tau)} \hat{\mathcal{L}}_{\tau}^{n} h_{\tau}\right)-\mu_{\sigma^{n}(\tau)}\left(f_{\sigma^{n}(\tau)}\right) \mu_{\tau}\left(h_{\tau}\right) \\
=\mu_{\sigma^{n}(\tau)}\left(f_{\sigma^{n}(\tau)} \hat{\mathcal{L}}_{\tau}^{n} \hat{h}_{\tau}\right) .
\end{aligned}
$$

Theorem 10.3 then gives that

$$
\left\|\hat{\mathcal{L}}_{\tau}^{n} \hat{h}_{\tau}\right\|_{\infty}=\left\|\hat{\mathcal{L}}_{\tau}^{n} h_{\tau}-\mu_{\tau}\left(h_{\tau}\right) \mathbb{1}_{\sigma^{n}(\tau)}\right\|_{\infty} \leq C_{h}(\omega)\left\|h_{\tau}\right\|_{\mathrm{BV}} \varkappa^{n}
$$

for each $n \in \mathbb{N}$. So by (11.2) and (11.3), we have

$$
\begin{aligned}
& \left|\mu_{\tau}\left(\left(f_{\sigma^{n}(\tau)} \circ T_{\tau}^{n}\right) h_{\tau}\right)-\mu_{\sigma^{n}(\tau)}\left(f_{\sigma^{n}(\tau)}\right) \mu_{\tau}\left(h_{\tau}\right)\right| \\
& \quad \leq \mu_{\sigma^{n}(\tau)}\left(\left|f_{\sigma^{n}(\tau)} \hat{\mathcal{L}}_{\tau}^{n} \hat{h}_{\tau}\right|\right) \leq C_{h}(\omega) \mu_{\sigma^{n}(\tau)}\left(\left|f_{\sigma^{n}(\tau)}\right|\right)\left\|h_{\tau}\right\|_{\mathrm{BV}} \varkappa^{n}
\end{aligned}
$$

for each $n \in \mathbb{N}$, which finishes the proof.

We are now able to prove Theorem 2.21.

Proof of Theorem 2.21. Theorem 2.21 now follows from Theorem 11.1 taking $p=-n$ and $p=0$.

Remark 11.2. We now show that the right-hand side of (11.1) does in fact converge to zero exponentially fast. First, note that for $f \in L_{\mu}^{1}(\Omega \times I)$ and $h \in \mathrm{BV}_{\Omega}^{l}(I)$ the Birkhoff Ergodic Theorem implies that for each $\delta>0$, there exists some measurable function $W_{f, h}$ : $\Omega \rightarrow(0, \infty)$ such that

$$
\left\|f_{\sigma^{n}(\omega)}\right\|_{L_{\mu_{\sigma^{n}}(\omega)}^{1}} \leq W_{f, h}(\omega) n \delta \leq W_{f, h}(\omega) e^{n \delta} \quad \text { and } \quad\left\|h_{\sigma^{-n}(\omega)}\right\|_{\infty} \leq W_{f, h}(\omega) n \delta \leq W_{f, h}(\omega) e^{n \delta} .
$$

Thus we may rewrite the conclusions of Theorem 11.1 (with $p=0$ and $p=-n$ ) to be

$$
\left|\mu_{\omega}\left(\left(f_{\sigma^{n}(\omega)} \circ T_{\omega}^{n}\right) h\right)-\mu_{\sigma^{n}(\omega)}\left(f_{\sigma^{n}(\omega)}\right) \mu_{\omega}(h)\right| \leq C_{h}(\omega) W_{f, h}(\omega) \kappa_{0}^{n}
$$

and

$$
\left|\mu_{\sigma^{-n}(\omega)}\left(\left(f_{\omega} \circ T_{\sigma^{-n}(\omega)}^{n}\right) h_{\sigma^{-n}(\omega)}\right)-\mu_{\omega}\left(f_{\omega}\right) \mu_{\sigma^{-n}(\omega)}\left(h_{\sigma^{-n}(\omega)}\right)\right| \leq C_{h}(\omega) W_{f, h}(\omega) \kappa_{0}^{n}
$$

for each $n \in \mathbb{N}$, where $0<\varkappa<\kappa_{0}=\varkappa \cdot e^{\delta}<1$ for some $\delta>0$ sufficiently small. Thus we see that the right-hand side of (11.1) does in fact go to zero exponentially fast.

Remark 11.3. If one were able to show that the measurable function $\Omega \ni \omega \mapsto C_{f}(\omega) \in \mathbb{R}^{+}$ were in fact $m$-integrable, then, following the proof of [40, Theorem 6.2], which is written in the setting where the coefficient $C_{f}(\omega)$ is independent of $f$ and $\omega$ and uses the method of Gordin and Liverani, we immediately obtain the Central Limit Theorem as a consequence of Theorem 11.1. For a more thorough treatment of stronger limit laws in the setting in which the coefficient $C_{f}(\omega)$ is independent of $f$ and $\omega$ see $[18,19]$. 
We are finally ready to prove the following proposition, which hinges on Theorem 11.1, and will complete the proof of Theorem 2.19.

Proposition 11.4. The measure $\mu$ defined in Proposition 9.3 is ergodic.

The proof of Proposition 11.4 is the same as the proof of Proposition 4.7 in [39], and is therefore left to the reader.

We now prove Theorem 2.19.

Proof of Theorem 2.19.

Part (1) of Theorem 2.19 follows from Proposition 3.1, Proposition 9.3, and Proposition 10.4.

Part (2) follows from Corollary 8.3, Proposition 9.3, Proposition 9.4, and Lemma 10.1.

Part (3) follows from Proposition 8.11, Proposition 9.3, Proposition 10.4, and Proposition 11.4.

\section{Relative Equilibrium States}

In this section we show that the random $T$-invariant probability measure $\mu$ defined in (9.9) is in fact the unique relative equilibrium state for the system, thus completing the proof of Theorem 2.23. Recall that $\mathcal{P}_{T}(\Omega \times I) \subseteq \mathcal{P}_{\Omega}(I)$ denotes the set of all $T$-invariant random probability measures on $I$, and for $\eta \in \mathcal{P}_{T}(\Omega \times I)$ the conditional information of the partition $Z_{\omega}^{*}$ given $T_{\omega}^{-1} \mathscr{B}$, with respect to $\eta_{\omega}$, is given by

$$
I_{\eta_{\omega}}=I_{\eta_{\omega}}\left[Z_{\omega}^{*} \mid T_{\omega}^{-1} \mathscr{B}\right]:=-\log g_{\eta, \omega}
$$

where

$$
g_{\eta, \omega}:=\sum_{Z \in Z_{\omega}^{*}} \mathbb{1}_{Z} E_{\eta_{\omega}}\left(\mathbb{1}_{Z} \mid T_{\omega}^{-1} \mathscr{B}\right)
$$

Remark 12.1. Note that $g_{\mu, \omega}=\hat{g}_{\omega}$, where $\hat{g}_{\omega}$ is the weight function defined in (8.49). To see this we note that

$$
E_{\mu_{\omega}}\left(\mathbb{1}_{Z} \mid T_{\omega}^{-1} \mathscr{B}\right)=\left.\hat{g}_{\omega}\right|_{Z}
$$

for each $Z \in Z_{\omega}^{*}$ since we have

$$
\hat{\mathcal{L}}_{\omega} \mathbb{1}_{Z}=\left.\hat{g}_{\omega}\right|_{Z}
$$

We now show that the $T$-invariant random measure $\mu$ defined in Proposition 9.3 is a relative equilibrium state, where we have followed the approach of [33] using the conditional information $I_{\mu_{\omega}}$ rather than the entropy $h_{\mu}(T)$, which for countable partitions may be infinite.

Lemma 12.2. The $T$-invariant random measure $\mu$ defined by (9.9) is a relative equilibrium state, i.e.

$$
\mathcal{E} P(\varphi)=\int_{\Omega}\left(\int_{I} I_{\mu_{\omega}}+\varphi_{\omega} d \mu_{\omega}\right) d m(\omega) .
$$

Proof. In light of Remark 12.1 and (8.49), the conditional information of $Z_{\omega}^{*}$ given $T_{\omega}^{-1} \mathscr{B}$, with respect to $\mu_{\omega}$, is given by

$$
I_{\mu_{\omega}}=-\log g_{\mu, \omega}=-\log \hat{g}_{\omega}=-\hat{\varphi}_{\omega} .
$$


Thus, we can write

$$
\begin{aligned}
\int_{I} I_{\mu_{\omega}}+\varphi_{\omega} d \mu_{\omega} & =\int_{I}-\hat{\varphi}_{\omega}+\varphi_{\omega} d \mu_{\omega} \\
& =\log \lambda_{\omega}+\int_{I} q_{\sigma(\omega)} \circ T_{\omega}-q_{\omega} d \mu_{\omega} \\
& =\log \lambda_{\omega}+\int_{I} q_{\sigma(\omega)} d \mu_{\sigma(\omega)}-\int_{I} q_{\omega} d \mu_{\omega}
\end{aligned}
$$

As $m$ is $\sigma$-invariant, integrating over $\Omega$ completes the proof.

In the following lemma we prove a variational principle for $T$-invariant random measures on $\mathcal{J}$.

Lemma 12.3. For all $\eta \in \mathcal{P}_{T}(\mathcal{J})$, the collection of all $T$-invariant random probability measures supported on $\mathcal{J}$, we have

$$
\log \lambda_{\omega} \geq \int_{X_{\omega}} I_{\eta, \omega}+\varphi_{\omega} d \eta_{\omega}
$$

for each $\omega \in \Omega$. Consequently, we have

$$
\mathcal{E} P(\varphi) \geq \int_{\Omega}\left(\int_{X_{\omega}} I_{\eta_{\omega}}+\varphi_{\omega} d \eta_{\omega}\right) d m(\omega)
$$

where equality holds if and only if $\eta_{\omega}=\mu_{\omega}$ for $m$-a.e. $\omega \in \Omega$.

Proof. Let $\eta \in \mathcal{P}_{T}(\mathcal{J})$. Then

$$
\begin{aligned}
I_{\eta_{\omega}}+\varphi_{\omega} & =-\log g_{\eta, \omega}+\varphi_{\omega} \\
& =-\log g_{\eta, \omega}+\log \hat{g}_{\omega}+\log \lambda_{\omega}-\log q_{\omega}+\log q_{\sigma(\omega)} \circ T_{\omega} \\
& =\log \frac{\hat{g}_{\omega}}{g_{\eta, \omega}}+\Phi_{\omega},
\end{aligned}
$$

where

$$
\Phi_{\omega}:=\log \lambda_{\omega}-\log q_{\omega}+\log q_{\sigma(\omega)} \circ T_{\omega} .
$$

Noting that $\eta_{\omega}\left(\Phi_{\omega}\right)=\log \lambda_{\omega}$, we see that to prove (12.1) it suffices to show that

$$
\int_{X_{\omega}} \log \frac{\hat{g}_{\omega}}{g_{\eta, \omega}} d \eta_{\omega} \leq 0
$$

Now, let $\mathcal{F}_{\omega}$ denote the set of bounded functions $f: X_{\omega} \rightarrow \mathbb{R}$ which are supported in a finite number of intervals of $\mathcal{Z}_{\omega}^{(1)}$. Let $\mathcal{L}_{\eta, \omega}: \mathcal{F}_{\omega} \rightarrow \mathcal{F}_{\sigma(\omega)}$ be given by

$$
\mathcal{L}_{\eta, \omega} f(x)=\sum_{y \in T_{\omega}^{-1}(x)} g_{\eta, \omega}(y) f(y), \quad f \in \mathcal{F}_{\omega}, x \in X_{\sigma(\omega)} .
$$

To complete the proof of (12.1) we prove the following claim.

\section{Claim 12.3.1.}

(i) For all $f \in \mathcal{F}_{\omega}$ we have $\eta_{\sigma(\omega)}\left(\mathcal{L}_{\eta, \omega}(f)\right)=\eta_{\omega}(f)$,

(ii) $\log ^{+} \frac{\hat{g}_{\omega}}{g_{\eta, \omega}} \in L_{\eta_{\omega}}^{1}\left(X_{\omega}\right)$ for each $\omega \in \Omega$, 
(iii) $\int_{X_{\omega}} \log \frac{\hat{g}_{\omega}}{g_{\eta, \omega}} \leq 0$.

Proof. We begin by noting that we may write

$$
\mathcal{L}_{\eta, \omega}(f)=\sum_{Z \in Z_{\omega}^{(1)}}\left(f \cdot \mathbb{1}_{Z} \cdot g_{\eta, \omega}\right) \circ T_{\omega, Z}^{-1}
$$

To prove (i) it suffices to prove the claim holds for the function $\mathbb{1}_{A}$ for some set $A$ which is contained in a finite number of partition elements. To that end, using (12.4) and the $T$-invariance of $\eta$, we calculate

$$
\begin{aligned}
\eta_{\sigma(\omega)}\left(\mathcal{L}_{\eta, \omega} \mathbb{1}_{A}\right) & =\eta_{\sigma(\omega)}\left(\sum_{Z \in \mathcal{Z}_{\omega}^{(1)}}\left(\mathbb{1}_{A} \mathbb{1}_{Z} g_{\eta, \omega}\right) \circ T_{\omega, Z}^{-1}\right) \\
& =\eta_{\omega}\left(\sum_{Z \in Z_{\omega}^{(1)}} \mathbb{1}_{A} \mathbb{1}_{Z} g_{\eta, \omega}\right) \\
& =\sum_{Z \in \mathcal{Z}_{\omega}^{(1)}} \eta_{\omega}\left(\mathbb{1}_{A} \mathbb{1}_{Z} \sum_{Y \in \mathcal{Z}_{\omega}^{(1)}}\left(\mathbb{1}_{Y} E_{\eta_{\omega}}\left(\mathbb{1}_{Y} \mid T_{\omega}^{-1} \mathscr{B}\right)\right)\right) \\
& =\sum_{Z \in \mathcal{Z}_{\omega}^{(1)}} \eta_{\omega}\left(\mathbb{1}_{A} \mathbb{1}_{Z} E_{\eta_{\omega}}\left(\mathbb{1}_{Z} \mid T_{\omega}^{-1} \mathscr{B}\right)\right) \\
& =\sum_{Z \in \mathcal{Z}_{\omega}^{(1)}} \eta_{\omega}\left(\mathbb{1}_{A} \mathbb{1}_{Z}\right)=\eta_{\omega}\left(\mathbb{1}_{A}\right) .
\end{aligned}
$$

To prove (ii) we first note that $g_{\eta, \omega}>0 \eta_{\omega}$-a.e. since, if $\left.g_{\eta, \omega}\right|_{A}=0$ for some subset $A \subseteq X_{\omega}$, then $\mathcal{L}_{\eta, \omega} \mathbb{1}_{A}=0$, and in light of (i) we would have $\eta_{\omega}(A)=0$.

Enumerate the intervals of $Z_{\omega}^{(1)}$ by $Z_{j}$ for $j \in \mathbb{N}$. Since $\log g_{\eta, \omega}>-\infty \eta_{\omega}$-a.e. and $\sup \hat{g}_{\omega}<\infty$ (by assumption (SP3), (3.5), and Proposition 8.10), we set

$$
F_{\omega}(n)(x):= \begin{cases}1 & \text { if } x \in Z_{1} \cup \cdots \cup Z_{n} \text { and }\left|\log \frac{\hat{g}_{\omega}}{g_{\eta, \omega}}(x)\right|<n \\ 0 & \text { otherwise, }\end{cases}
$$

and

$$
\chi_{\omega}^{+}:=\left\{x \in X_{\omega}: \log \frac{\hat{g}_{\omega}}{g_{\eta, \omega}}(x) \geq 0\right\} .
$$

Since $\hat{g}_{\omega} \geq g_{\eta, \omega}$ on $\chi_{\omega}^{+}$, we have

$$
0 \leq \mathcal{L}_{\eta, \omega}\left(F_{\omega}(n) \mathbb{1}_{\chi_{\omega}^{+}}\right) \leq \hat{\mathcal{L}}_{\omega}\left(F_{\omega}(n) \mathbb{1}_{\chi_{\omega}^{+}}\right) \leq \hat{\mathcal{L}}_{\omega} \mathbb{1}_{\omega}=\mathbb{1}_{\sigma(\omega)}
$$

In light of the definitions of $\hat{\mathcal{L}}_{\omega},(8.50)$, and $\mathcal{L}_{\eta, \omega},(12.3)$, we see that

$$
\mathcal{L}_{\eta, \omega}\left(f \frac{\hat{g}_{\omega}}{g_{\eta, \omega}}\right)(x)=\hat{\mathcal{L}}_{\omega} f(x) .
$$


Now, using (12.5), (i), the fact that $\log x \leq x-1$ for $x>0,(12.7)$, and (12.6) we thus get that

$$
\begin{aligned}
\int_{X_{\omega}} \mathbb{1}_{\chi_{\omega}^{+}} \log \frac{\hat{g}_{\omega}}{g_{\eta, \omega}} d \eta_{\omega} & =\lim _{n \rightarrow \infty} \int_{X_{\omega}} F_{\omega}(n) \mathbb{1}_{\chi_{\omega}^{+}} \log \frac{\hat{g}_{\omega}}{g_{\eta, \omega}} d \eta_{\omega} \\
& =\lim _{n \rightarrow \infty} \int_{X_{\sigma(\omega)}} \mathcal{L}_{\eta, \omega}\left(F_{\omega}(n) \mathbb{1}_{\chi_{\omega}^{+}} \log \frac{\hat{g}_{\omega}}{g_{\eta, \omega}}\right) d \eta_{\sigma(\omega)} \\
& \leq \lim _{n \rightarrow \infty} \int_{X_{\sigma(\omega)}} \mathcal{L}_{\eta, \omega}\left(F_{\omega}(n) \mathbb{1}_{\chi_{\omega}^{+}}\left(\frac{\hat{g}_{\omega}}{g_{\eta, \omega}}-1\right)\right) d \eta_{\sigma(\omega)} \\
& =\lim _{n \rightarrow \infty} \int_{X_{\sigma(\omega)}}\left(\mathcal{L}_{\eta, \omega}\left(F_{\omega}(n) \mathbb{1}_{\chi_{\omega}^{+}} \frac{\hat{g}_{\omega}}{g_{\eta, \omega}}\right)-\mathcal{L}_{\eta, \omega}\left(F_{\omega}(n) \mathbb{1}_{\chi_{\omega}^{+}}\right)\right) d \eta_{\sigma(\omega)} \\
& =\lim _{n \rightarrow \infty} \int_{X_{\sigma(\omega)}}\left(\hat{\mathcal{L}}_{\omega}\left(F_{\omega}(n) \mathbb{1}_{\chi_{\omega}^{+}}\right)-\mathcal{L}_{\eta, \omega}\left(F_{\omega}(n) \mathbb{1}_{\chi_{\omega}^{+}}\right)\right) d \eta_{\sigma(\omega)} \\
& \leq 1 .
\end{aligned}
$$

Hence, we have that $\chi_{\omega}^{+} \log \left(\hat{g}_{\omega} / g_{\eta, \omega}\right) \in L_{\eta_{\omega}}^{1}\left(X_{\omega}\right)$, which proves (ii).

Now to prove claim (iii), we note that if

$$
\int_{X_{\omega}} \log \frac{\hat{g}_{\omega}}{g_{\eta, \omega}} d \eta_{\omega}=-\infty
$$

then we are done. Otherwise, by (ii), we have $\log \left(\hat{g}_{\omega} / g_{\eta, \omega}\right) \in L_{\eta_{\omega}}^{1}\left(X_{\omega}\right)$. Repeating the calculation from the beginning of the previous display block of equations to (12.8) without the characteristic function $\mathbb{1}_{\chi_{\omega}^{+}}$and then using (8.51) and (i) gives

$$
\begin{aligned}
\int_{X_{\omega}} \log \frac{\hat{g}_{\omega}}{g_{\eta, \omega}} d \eta_{\omega} & \leq \lim _{n \rightarrow \infty} \int_{X_{\sigma(\omega)}} \hat{\mathcal{L}}_{\omega}\left(F_{\omega}(n)\right) d \eta_{\sigma(\omega)}-\lim _{n \rightarrow \infty} \int_{X_{\sigma(\omega)}} \mathcal{L}_{\eta, \omega}\left(F_{\omega}(n)\right) d \eta_{\sigma(\omega)} \\
& \leq \int_{X_{\sigma(\omega)}} \hat{\mathcal{L}}_{\omega}\left(\mathbb{1}_{\omega}\right) d \eta_{\sigma(\omega)}-\lim _{n \rightarrow \infty} \int_{X_{\sigma(\omega)}} \mathcal{L}_{\eta, \omega}\left(F_{\omega}(n)\right) d \eta_{\sigma(\omega)} \\
& \leq 1-\lim _{n \rightarrow \infty} \int_{X_{\omega}} F_{\omega}(n) d \eta_{\omega}=0 .
\end{aligned}
$$

Thus, we have that

$$
\int_{X_{\omega}} I_{\eta_{\omega}}+\varphi_{\omega} d \eta_{\omega}=\int_{X_{\omega}} \Phi_{\omega} d \eta_{\omega}+\int_{X_{\omega}} \log \frac{\hat{g}_{\omega}}{g_{\eta, \omega}} d \eta_{\omega} \leq \log \lambda_{\omega},
$$

which finishes the proof of (12.1). Thus, (12.2) follows by integrating over $\Omega$.

Finally, we note that equality holds if and only if $\hat{g}_{\omega}=g_{\eta, \omega} \eta_{\omega}$-almost everywhere on $X_{\omega}$, which implies that $\hat{\mathcal{L}}_{\omega} f(x)=\mathcal{L}_{\eta, \omega} f(x)$ for $\eta_{\omega}$-a.e. $x \in X_{\omega}$ and all $f \in \operatorname{BV}\left(X_{\omega}\right)$. Thus, applying Theorem 10.3 with $p=0$, we have

$$
\begin{aligned}
\left|\eta_{\omega}(f)-\mu_{\omega}(f)\right| & =\left|\eta_{\sigma^{n}(\omega)}\left(\mathcal{L}_{\eta, \omega}^{n} f\right)-\mu_{\omega}(f)\right| \\
& =\left|\eta_{\sigma^{n}(\omega)}\left(\hat{\mathcal{L}}_{\omega}^{n} f\right)-\mu_{\omega}(f)\right| \\
& =\left|\eta_{\sigma^{n}(\omega)}\left(\hat{\mathcal{L}}_{\omega}^{n} f-\mu_{\omega}(f) \mathbb{1}_{\sigma^{n}(\omega)}\right)\right|
\end{aligned}
$$




$$
\begin{aligned}
& \leq\left|\eta_{\sigma^{n}(\omega)}\left(\left\|\hat{\mathcal{L}}_{\omega}^{n} f-\mu_{\omega}(f) \mathbb{1}_{\sigma^{n}(\omega)}\right\|_{\infty}\right)\right| \\
& \leq C_{f}(\omega)\|f\|_{\mathrm{BV}} \varkappa^{n}
\end{aligned}
$$

for each $n \in \mathbb{N}$ and every $f \in \operatorname{BV}\left(X_{\omega}\right)$. Thus we must in fact have that $\eta_{\omega}(f)=\mu_{\omega}(f)$ for each $f \in \mathrm{BV}\left(X_{\omega}\right)$, and thus $\eta_{\omega}=\mu_{\omega}$ on a dense set of $\mathcal{C}\left(X_{\omega}\right)$. We conclude finally that $\mu_{\omega}=\eta_{\omega}$ for $m$-a.e. $\omega \in \Omega$, which completes the proof.

To finish the proof of Theorem 2.23, we now extend the variational principle on $\mathcal{J}$, proved in Lemma 12.3, to $\Omega \times I$.

Lemma 12.4. For any $\eta \in \mathcal{P}_{T}(\Omega \times I)$ we have

$$
\mathcal{E} P(\varphi) \geq \int_{\Omega}\left(\int_{I} I_{\eta_{\omega}}+\varphi_{\omega} d \eta_{\omega}\right) d m(\omega)
$$

Moreover, equality holds if and only if $\eta_{\omega}=\mu_{\omega}$ for $m$-a.e. $\omega \in \Omega$.

Proof. Suppose $\eta \in \mathcal{P}_{T}(\Omega \times I)$ is a $T$-invariant random probability measure on $I$, and suppose that for each $\omega \in \Omega$ we have that

$$
\eta_{\omega}=c_{\omega} \eta_{\omega}^{c}+a_{\omega} \eta_{\omega}^{a}
$$

where $\eta_{\omega}^{c}$ is a non-atomic probability measure on $I, \eta_{\omega}^{a}$ is a purely atomic probability measure on $I$, and $c_{\omega}+a_{\omega}=1$. As $\eta_{\omega}^{c}$ is non-atomic, we must have that $\eta_{\omega}^{c}\left(\mathcal{S}_{\omega}\right)=0$ as the singular set $\mathcal{S}_{\omega}$, defined by $(2.1)$, is countable. Without loss of generality, we suppose that

$$
\int_{\Omega} a_{\omega} d m(\omega)>0
$$

as otherwise the lemma would follow from Lemma 12.3.

We easily see that $I_{\eta_{\omega}^{a}, \omega}=0 \eta_{\omega}^{a}$-a.e, and since $\eta_{\omega}^{a}$ and $\eta_{\omega}^{c}$ are singular, we can choose $I_{\eta_{\omega}^{a}, \omega}=0 \eta_{\omega}^{c}$-a.e and $I_{\eta_{\omega}^{c}, \omega}=0 \eta_{\omega}^{a}$-a.e. Therefore, we have that

$$
I_{\eta, \omega}=I_{\eta^{c}, \omega}+I_{\eta^{a}, \omega}=I_{\eta^{c}, \omega},
$$

and hence, applying Lemma 12.3 gives

$$
\begin{aligned}
\int_{\Omega}\left(\int_{I} I_{\eta_{\omega}}+\varphi_{\omega} d \eta_{\omega}\right) d m(\omega) & =\int_{\Omega} c_{\omega} \int_{I} I_{\eta^{c}, \omega}+\varphi_{\omega} d \eta_{\omega}^{c} d m(\omega)+\int_{\Omega} a_{\omega} \int_{I} \varphi_{\omega} d \eta_{\omega}^{a} d m(\omega) \\
& \leq \int_{\Omega} c_{\omega} \log \lambda_{\omega} d m(\omega)+\int_{\Omega} a_{\omega} \int_{I} \varphi_{\omega} d \eta_{\omega}^{a} d m(\omega) .
\end{aligned}
$$

Since $\eta$ is a random probability measure we have that $\eta_{\omega}^{a}\left(\varphi_{\omega}\right) \in L_{m}^{1}(\Omega)$. Thus, applying the Birkhoff Ergodic Theorem, (CP1), and Lemma 10.1 for each $\varepsilon>0$ we can find $n \in \mathbb{N}$ so large that

$$
\begin{aligned}
\int_{\Omega} \int_{I} \varphi_{\omega} d \eta_{\omega}^{a} d m(\omega)-\varepsilon & \leq \frac{1}{n} S_{n, \sigma}\left(\eta_{\omega}^{a}\left(\varphi_{\omega}\right)\right) \\
& =\frac{1}{n}\left(\eta_{\omega}^{a}\left(\varphi_{\omega}\right)+\cdots+\eta_{\omega}^{a}\left(\varphi_{\sigma^{n-1}(\omega)} \circ T_{\omega}^{n-1}\right)\right) \\
& =\frac{1}{n} \eta_{\omega}^{a}\left(\log g_{\omega}^{(n)}\right) \leq \frac{1}{n} \log \left\|g_{\omega}^{(n)}\right\|_{\infty} \\
& <\frac{1}{n} \log \inf \mathcal{L}_{\omega}^{n} \mathbb{1}_{\omega} \leq \mathcal{E} P(\varphi)+\varepsilon .
\end{aligned}
$$


As this holds for every $\varepsilon>0$ we must in fact have

$$
\int_{\Omega} a_{\omega} \int_{I} \varphi_{\omega} d \eta_{\omega}^{a} d m(\omega)<\int_{\Omega} a_{\omega} \log \lambda_{\omega} d m(\omega) .
$$

Inserting (12.11) into (12.10) we see that then

$$
\begin{aligned}
\int_{\Omega} \int_{I} I_{\eta_{\omega}}+\varphi_{\omega} d \eta_{\omega} d m(\omega) & <\int_{\Omega} c_{\omega} \log \lambda_{\omega} d m(\omega)+\int_{\Omega} a_{\omega} \log \lambda_{\omega} d m(\omega) \\
& =\int_{\Omega} \log \lambda_{\omega} d m(\omega)
\end{aligned}
$$

Thus, integrating with respect to $m$, we see that if $\int_{\Omega} a_{\omega} d m(\omega)>0$, then

$$
\varepsilon P(\varphi)>\int_{\Omega}\left(\int_{I} I_{\eta_{\omega}}+\varphi_{\omega} d \eta_{\omega}\right) d m(\omega) .
$$

Thus for equality to hold in (12.9) for $\eta \in \mathcal{P}_{T}(\Omega \times I)$ with $\eta_{\omega}=c_{\omega} \eta_{\omega}^{c}+a_{\omega} \eta_{\omega}^{a}$, we see that we must have

$$
\int_{\Omega} a_{\omega} d m(\omega)=0
$$

and thus, in light of Lemma 12.3, we must have that $\eta_{\omega}=\eta_{\omega}^{c}=\mu_{\omega}$ for $m$-a.e. $\omega \in \Omega$.

Proof of Theorem 2.23. Theorem 2.23 now follows from Lemmas 12.2, 12.3, and 12.4.

\section{EXAMPLES}

In this section we present several examples for which our general theory applies. In particular, we treat random $\beta$-transformations, randomly translated random $\beta$-transformations, random infinitely branched Gauss-Renyi maps, random maps with non-uniform expansion (specifically random systems with Pomeau-Manneville maps and random maps with contracting branches), and a broad class of random Lasota-Yorke maps.

Concerning examples, we would like to point out that $\beta$-transformations are an important class of systems, among the most studied in ergodic theory since the pioneering work of Parry [42]; they serve also as a prototype to test theories of random perturbations in the non-stationary setting. For instance limit theorems for sequential expanding dynamical systems were rigorously proved in [13] for $\beta$-transformations using quite advanced technical tools. Similar care is necessary to treat the quenched case we deal with and we will show that the methods we use can also be generalized to wider classes of map like the Lasota-Yorke ones.

As stated in Remark 4.8, it suffices to check conditions (M5') and (M6') rather than (M5) and (M6). Thus, in the general strategy of checking that the conditions are satisfied for a particular example, one must find the number $N_{*}$ as defined in (4.6). For computational ease we have selected some of our examples (Sections 13.2, 13.4, and 13.5) so that we will have $N_{*}=1$. However, we would like to point out that one could deal with the case of $N_{*}>1$ using similar arguments as the ones we present here.

For the rest of this section, we assume $\Omega$ is a topological space, $m$ is a complete probability measure on its Borel sigma-algebra and $\sigma:(\Omega, m) \rightarrow(\Omega, m)$ is a continuous, ergodic, invertible, probability-preserving map. 
13.1. Random $\beta$-Transformations (I). Let $\beta: \Omega \rightarrow \mathbb{R}_{+}$, given by $\omega \mapsto \beta_{\omega}$, be an $m$ continuous function. That is, there is a partition of $\Omega(\bmod m)$ into at most countably many Borel sets $\Omega_{1}, \Omega_{2}, \ldots$ such that $\beta$ is constant on each $\Omega_{j}$, say $\left.\beta\right|_{\Omega_{j}}=\beta_{j}$. Consider a random beta transformation $T_{\omega}:[0,1] \rightarrow[0,1]$ given by

$$
T_{\omega}(x)=\beta_{\omega} x \quad(\bmod 1) \text {. }
$$

Lemma 13.1. Assume $\beta$ satisfies the expanding on average condition $E:=\int_{\Omega} \log \beta_{\omega} d m>$ 0. Then, the system has the random covering property (RC) (Definition 2.7).

Proof. Let $J \subset I$ be an interval. The expanding on average condition, combined with the Birkhoff ergodic theorem, ensures that for $m$ a.e. $\omega \in \Omega$, in finite time, say $\ell-1 \in \mathbb{N}$, the image of $J$ contains a discontinuity in its interior. Hence, at the following iterate, it contains an interval of the form $[0, a]$ for some $a>0$. After this, it takes at most

$$
\tilde{n}(\omega)=\min \left\{n \geq 1: \beta_{\sigma^{\ell} \omega}^{(n)} a \geq 1\right\}
$$

more iterates for the images of $J$ to cover $I$, where $\beta_{\omega}^{(n)}=\beta_{\omega} \beta_{\sigma \omega} \ldots \beta_{\sigma^{n-1} \omega}$. Once again, this time is finite for $m$ a.e. $\omega \in \Omega$ because of the expanding on average condition and Birkhoff ergodic theorem.

Lemma 13.2. Let $\Phi: \Omega \rightarrow B V(I)$ be an $m$-continuous function ${ }^{11}$. Let $\varphi: \Omega \times I \rightarrow \mathbb{R}$ be given by $\varphi_{\omega}:=\varphi(\omega, \cdot)=\Phi(\omega)$. Then $g_{\omega}=e^{\varphi_{\omega}} \in B V(I)$ for $m$ a.e. $\omega \in \Omega$. Assume there exist $\varphi_{-}, \varphi_{+} \in \mathbb{R}$ such that $\varphi_{-} \leq \varphi(\omega, x)<\varphi_{+}$, and that

$$
\varphi_{+}-\varphi_{-}<\int_{\Omega} \log \left\lfloor\beta_{\omega}\right\rfloor d m=: E_{-} .
$$

Furthermore, assume the integrability condition $\int_{\Omega} \log \beta_{\omega} d m<\infty^{12}$. Finally, assume that either

(i) $g_{\omega}=\left|T_{\omega}^{\prime}\right|^{-t}$ for some $t \geq 0$,

(ii) condition $(\mathrm{P} 1)$ holds for the dynamical partitions $\mathcal{P}_{\omega, n}(0,1)=\mathcal{Z}_{\omega}^{(n)}$.

Then $\varphi$ is a summable contracting potential for $\left(\Omega, \sigma, m,\left(T_{\omega}\right)_{\omega \in \Omega}\right)$, and conditions (GP) and (M1)-(M4) are satisfied.

Proof. Condition (GP) holds because the system is expanding on average. The summability conditions (Definition 2.2) are straightforward to check. Indeed, (SP2) is a hypothesis. (SP1) and (SP3) hold because $\varphi$ is bounded and the partitions are finite. When $g_{\omega}=\left|T_{\omega}^{\prime}\right|^{-t}$ for some $t \geq 0$, (P1) holds with $\hat{\alpha}=0$ because $g_{\omega}^{(n)}$ is constant a.e. In general, (P2) holds with $\hat{\gamma}=1$ because $\mathcal{P}_{\omega, n}(0,1)$ coincides with the dynamical partition $z_{\omega}^{(n)}$. (M1) and (M2) follow from $m$-continuity; see e.g. [21]. (M3) holds because $e^{\varphi_{-}} \leq S_{\omega}^{(1)} \leq\left\lceil\beta_{\omega}\right\rceil e^{\varphi_{+}}$, where $\left\lceil\beta_{\omega}\right\rceil$ is the least integer greater than or equal to $\beta$ and also the cardinality of $z_{\omega}^{*}$. Thus,

$$
\int_{\Omega}\left|\log S_{\omega}^{(1)}\right| d m \leq \max \left\{\left|\varphi_{-}\right|, \int_{\Omega} \log \left\lceil\beta_{\omega}\right\rceil d m+\left|\varphi_{+}\right|\right\}<\infty .
$$

Notice that inf $\mathcal{L}_{\omega} \mathbb{1}_{\omega} \geq n\left(T_{\omega}\right) e^{\varphi_{-}}$, where $n\left(T_{\omega}\right)=\min \left\{\# T_{\omega}^{-1}(x): x \in I\right\}$ is the minimum number of preimages of a point $x \in I$ under $T_{\omega}$. Since $T_{\omega}$ is a $\beta$-transformation,

$$
n\left(T_{\omega}\right)=\#\left(T_{\omega}\right)^{-1}(1)=\left\lfloor\beta_{\omega}\right\rfloor,
$$

\footnotetext{
${ }^{11}$ Without loss of generality, we assume the partitions on which $\beta$ and $\Phi$ are constant coincide.

${ }^{12}$ Note that this condition is equivalent with the assumption that $\int_{\Omega} \log \left\lceil\beta_{\omega}\right\rceil d m<\infty$
} 
the largest integer less than or equal to $\beta_{\omega}$. Thus, in view of Remark 2.16, with $N_{1}=N_{3}=1$, in order to show the potential is contracting (Definition 2.15), it is sufficient to have that

$$
\varphi_{+}<\varphi_{-}+\int_{\Omega} \log \left\lfloor\beta_{\omega}\right\rfloor d m,
$$

as assumed.

Furthermore, the condition $E_{-}>\varphi_{+}-\varphi_{-}$ensures that $\left\lfloor\beta_{\omega}\right\rfloor \geq 1$ for $m$ a.e. $\omega \in \Omega$. This implies that $\beta_{\omega} \geq 1$ for $m$ a.e. $\omega \in \Omega$, and so $\log \inf \mathcal{L}_{\omega} \mathbb{1} \geq \varphi_{-}$. This yields (M4).

Remark 13.3. Note that the hypotheses of Lemma 13.2 imply those of Lemma 13.1.

Lemma 13.4. Under the hypotheses of Lemma 13.2, Theorems 2.19 - 2.23 apply provided

$$
M_{\omega, N_{*}} \in L_{m}^{1}(\Omega) \quad \text { and } \quad \log b_{\omega}^{\left(M_{\omega, N_{*}}\right)} \in L_{m}^{1}(\Omega),
$$

where $N_{*}$ is defined in (4.6), $M_{\omega, N_{*}}=\max _{Z \in \mathcal{Z}_{\omega}^{\left(N_{*}\right)}} M_{\omega}(Z)$ is the maximum covering time for branches of $T_{\omega}^{N_{*}}$ and $b_{\omega}:=\left\lceil\beta_{\omega}\right\rceil$ is the least integer greater than or equal to $\beta_{\omega}$.

Proof. In view of Lemmas 13.1 and 13.2, in order to satisfy the hypotheses of Theorems 2.19 -2.23 , it is enough to verify the conditions (M5') and (M6'), namely

$$
\log \left\|\mathcal{L}_{\omega}^{M_{\omega, N_{*}}} \mathbb{1}\right\|_{\infty} \in L_{m}^{1}(\Omega), \quad \log \operatorname{ess} \inf g_{\omega}^{\left(M_{\omega, N_{*}}\right)} \in L_{m}^{1}(\Omega) .
$$

Let us assume $\varphi, \varphi_{-}, \varphi_{+} \in \mathbb{R}$ are as in Lemma 13.2. Then

$$
M_{\omega, N_{*}} \varphi_{-} \leq \log \operatorname{ess} \inf g_{\omega}^{\left(M_{\omega, N_{*}}\right)} \leq \log \operatorname{ess} \sup g_{\omega}^{\left(M_{\omega, N_{*}}\right)} \leq M_{\omega, N_{*}} \varphi_{+} .
$$

Also, recalling that the map $x \mapsto \beta_{\omega} x(\bmod 1)$ has $b_{\omega}=\left\lceil\beta_{\omega}\right\rceil$ branches, we have that

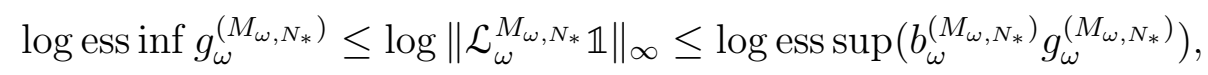

where $b_{\omega}^{(n)}=\prod_{j=0}^{n-1} b_{\sigma^{j} \omega}$. Therefore,

$$
M_{\omega, N_{*}} \varphi_{-} \leq \log \left\|\mathcal{L}_{\omega}^{M_{\omega, N_{*}}} \mathbb{1}\right\|_{\infty} \leq \log \left(b_{\omega}^{\left(M_{\omega, N_{*}}\right)}\right)+M_{\omega, N_{*}} \varphi_{+} .
$$

Thus, under the hypotheses of the lemma, conditions (13.2) hold.

In what follows, we discuss explicit conditions which will hold if (13.1) holds. Notice that once the first condition of (13.1) holds, the second one also holds provided there exists $\beta_{+} \in \mathbb{R}$ such that $\beta_{\omega} \leq \beta_{+}$. Let us investigate the first condition of (13.1) in more detail.

Let us first discuss the case $N_{*}=1$. That is, assume (4.6) holds with $N_{*}=1$. Let

$$
s_{\omega}=\frac{\left\{\beta_{\omega}\right\}_{+}}{\beta_{\omega}}
$$

where $\{\beta\}_{+}$denotes the fractional part of $\beta$ if $\beta \notin \mathbb{N}$ and $\{\beta\}_{+}=1$ if $\beta \in \mathbb{N}$. Then, $s_{\omega}$ is the length of the shortest branch of the monotonicity partition of $T_{\omega}$. Note that all but at most one of the elements $Z \in Z_{\omega}^{*}$ have the property that $T_{\omega}(Z)=I$, and the remaining one is of the form $Z_{\omega}=\left[1-s_{\omega}, 1\right]$. Thus, it takes at most $\tau_{\omega}\left(s_{\omega}\right)$ iterates for each $Z \in Z_{\omega}^{*}$ to cover $I$, where

$$
\tau_{\omega}(s):=\min \left\{n \geq 1: \beta_{\omega}^{(n)} s \geq 1\right\} .
$$

Then, the covering time satisfies $M_{\omega, 1}=\tau_{\omega}\left(s_{\omega}\right)$. The fact that $\tau_{\omega}(s)<\infty$ for every $s>0$ and $m$ a.e. $\omega \in \Omega$ follows from the expansion on average property. Thus, in this case, (13.1) holds provided

$$
\int_{\Omega} \tau_{\omega}\left(s_{\omega}\right) d m<\infty
$$


One may construct examples (as done in the following subsection, where the upper bound on $\beta$ is also relaxed) when this occurs, for example by controlling the occurrence of small pieces $s_{\omega}$ and small values of $\beta_{\omega}$.

When (4.6) holds with $N_{*}>1$, (13.1) holds provided

$$
\int_{\Omega} \tau_{\omega}\left(s_{\omega, N_{*}}\right) d m<\infty
$$

where $s_{\omega, N_{*}}$ is length of the shortest branch of $z_{\omega}^{\left(N_{*}\right)}$. This length may be characterized inductively as follows: $s_{\omega, 1}=s_{\omega}$ is given by (13.3). Assuming $s_{\omega, k}$ has been computed,

$$
s_{\omega, k+1}=\min \left\{\frac{s_{\sigma^{k}(\omega), 1}}{\beta_{\omega}^{(k)}}, \frac{\left\{\beta_{\omega}^{(k+1)} s_{\omega, k}\right\}_{+}}{\beta_{\sigma^{k}(\omega)}}\right\} .
$$

13.2. Random $\beta$-Transformations (II). In this subsection we assume that there is some $\delta>0$ such that for $m$-a.e. $\omega \in \Omega$ we have

$$
\beta_{\omega} \in \bigcup_{k \in \mathbb{N}}[k+\delta, k+1] .
$$

We consider the potential $\varphi_{\omega}=-t \log \left|T_{\omega}^{\prime}\right|, t \geq 0$. Note that in this setting we have relaxed the uniform lower bound on $\varphi_{\omega}$ and the uniform upper bound on the number of branches of $T_{\omega}$ from the previous subsection.

Lemma 13.5. Under the above conditions, the time for an interval of monotonicity of $T_{\omega}$ to cover is no more than $1+\left\lceil\frac{-\log \delta}{\log (1+\delta)}\right\rceil$.

Proof. If the interval of monotonicity is a full branch it takes one iteration. Otherwise, we must be at the final (non-full) branch and therefore after one iteration we have an interval $[0, \delta)$. At each subsequent iteration this interval grows by a factor at least $1+\delta$. If the interval is cut at some future point, it means the interval has expanded across a full branch and we have covered. In the worst case, we repeatedly apply the map with least slope, namely $1+\delta$ and it takes $n$ further iterations beyond the first, where $\delta(1+\delta)^{n} \geq 1$. Solving for $n$ yields the claim.

Lemma 13.6. Under the m-continuity conditions of Lemma 13.2 and the conditions on the maps in this subsection, additionally assume that

$$
\int_{\Omega} \log \left\lfloor\beta_{\omega}\right\rfloor d m(\omega)>\log 3
$$

and

$$
\int_{\Omega} \log \left\lceil\beta_{\omega}\right\rceil d m(\omega)<\infty .
$$

Then $\varphi$ is a summable contracting potential, and the system satisfies conditions (M1)-(M4) and (M5')-(M6'). Thus Theorems 2.19 - 2.23 apply.

Proof. We first note that (SP1), (SP2), and hold since $g_{\omega}^{(1)}$ is constant for $m$-a.e. $\omega \in \Omega$ and that (SP3) holds since $S_{\omega}^{(1)}=\frac{\left\lceil\beta_{\omega}\right\rceil}{\beta_{\omega}^{t}}<\infty$. Now we note that we may take the finite partition $\mathcal{P}_{\omega, n}(0,1)=z_{\omega}^{(n)}$. Indeed, condition $(\mathrm{P} 1)$ holds with $\hat{\alpha}=0$ because $g_{\omega}^{(n)}$ is constant for $m$-a.e. $\omega \in \Omega$, and Since $z_{\omega}^{(n)}$ is finite for $m$-a.e. $\omega \in \Omega$, by Remark 2.9, (P2) holds with 
$\hat{\gamma}=1$. Conditions (M1) and (M2) hold by $m$-continuity, see [21], and (M3) holds since $\log S_{\omega}^{(1)}=\log \left\lceil\beta_{\omega}\right\rceil-t \log \beta_{\omega}$, using (13.7) and noting $\beta_{\omega} \geq 1+\delta$. Similarly, (M4) holds since

$$
\log \left\lfloor\beta_{\omega}\right\rfloor-t \log \beta_{\omega} \leq \log \inf \mathcal{L}_{\omega} 1 \leq S_{\omega}^{(1)},
$$

again using (13.7) and noting $\beta_{\omega} \geq 1+\delta$. To show $\varphi$ is contracting we apply (2.13) with $N_{1}=N_{3}=1$. One has

$$
\int \log \left|g_{\omega}\right|_{\infty} d m=-t \int \log \beta_{\omega} d m<\int \log \left\lfloor\beta_{\omega}\right\rfloor d m-t \int \log \beta_{\omega} d m \leq \int \log \inf \mathcal{L}_{\omega} \mathbb{1}_{\omega} d m,
$$

with the central inequality holding by (13.6).

For (M5') and (M6') we first show that $N_{*}=1$. The quantity $Q_{\omega}^{(1)}$ defined in (4.5) is bounded between $3 /\left\lceil\beta_{\omega}\right\rceil$ and $3 /\left\lfloor\beta_{\omega}\right\rfloor$. Recalling that $\beta_{\omega} \geq 1+\delta$ for $m$-a.e. $\omega \in \Omega$ and using (13.6)-(13.7), we obtain

$$
-\infty<\int_{\Omega} \log Q_{\omega}^{(1)} d m<0
$$

and therefore it follows from (4.6) that we may take $N_{*}=1$. By Remark 2.9 and the fact that $N_{*}=1$ we may check $\left(\mathrm{M}^{\prime}\right)$ and (M6') for $\mathcal{P}_{\omega, 1}=\mathcal{Z}_{\omega}^{(1)}$. Thus, to verify (M5') and (M6') we need to check that

$$
\min _{P \in Z_{\omega}^{(1)}} \log \inf _{P} g_{\omega}^{M_{\omega}(P)}=\min _{P \in Z_{\omega}^{(1)}} \inf _{P}-t \log \left|\left(T_{\omega}^{\left(M_{\omega}(P)\right)}\right)^{\prime}\right| \in L_{m}^{1}(\Omega)
$$

and

$$
\max _{P \in \mathcal{Z}_{\omega}^{(1)}} \log \left\|\mathcal{L}_{\omega}^{M_{\omega}(P)} \mathbb{1}_{\omega}\right\|_{\infty} \in L_{m}^{1}(\Omega),
$$

respectively. By Lemma 13.5,

$$
M_{\omega}(P) \leq 1+\left\lceil\frac{-\log \delta}{\log (1+\delta)}\right\rceil .
$$

Thus (13.8) will hold if

$$
\log \beta_{\omega}^{\left(1+\left\lceil\frac{-\log \delta}{\log (1+\delta)}\right\rceil\right)} \in L_{m}^{1}(\Omega),
$$

or equivalently, if $\int \log \beta_{\omega} d m(\omega)<\infty$, which is the case by (13.7).

Concerning (13.9),

$$
\begin{aligned}
\left|\log \mathcal{L}_{\omega}^{M_{\omega}(P)} \mathbb{1}_{\omega}\right| & \leq\left|\log \left(\frac{\left\lceil\beta_{\omega}\right\rceil}{\beta_{\omega}^{t}}\right)^{\left(M_{\omega}(P)\right)}\right| \\
& \leq\left|\log \left(\frac{\left\lceil\beta_{\omega}\right\rceil}{\beta_{\omega}^{t}}\right)^{\left(1+\left\lceil\frac{-\log \delta}{\log (1+\delta)}\right\rceil\right)}\right| \\
& =\left|\log \left\lceil\beta_{\omega}\right\rceil^{\left(1+\left\lceil\frac{-\log \delta}{\log (1+\delta)}\right\rceil\right)}-t \log \beta_{\omega}^{\left(1+\left\lceil\frac{-\log \delta}{\log (1+\delta)}\right\rceil\right)}\right| \\
& \leq(1+t)\left|\log \left\lceil\beta_{\omega}\right\rceil^{\left(1+\left\lceil\frac{-\log \delta}{\log (1+\delta)}\right\rceil\right)}\right| .
\end{aligned}
$$

As in the case of (M5') above, integrability of (13.10) is equivalent to $\int_{\Omega} \log \left\lceil\beta_{\omega}\right\rceil d m(\omega)<\infty$, which follows from (13.7). 
Remark 13.7. Note that we could replace the requirement that $\int_{\Omega} \log \left\lfloor\beta_{\omega}\right\rfloor d m(\omega)>\log 3$ in the previous lemma with the requirement that $\beta_{\omega}>1+\delta$ for $m$-a.e. $\omega \in \Omega$ at the expense of calculating the number $N_{*}>1$ to satisfy (CP2), and checking (M5') and (M6'). Furthermore, note that (M5') and (M6') are automatically satisfied in the setting of finitely many maps $T_{\omega}$.

13.3. Randomly Translated Random $\beta$-Transformations. Let $\alpha, \beta: \Omega \rightarrow \mathbb{R}_{+}$, be $m$ continuous functions such that $\beta_{\omega}$ is essentially bounded and satisfies the expanding condition $\operatorname{ess~}_{\inf } \omega \in \Omega \beta_{\omega}=2+\delta$, for some $\delta>0$. Consider the random beta transformation $T_{\omega}:[0,1] \rightarrow$ $[0,1]$ given by

$$
T_{\omega}(x)=\beta_{\omega} x+\alpha_{\omega} \quad(\bmod 1) .
$$

Lemma 13.8. Let $\varphi: \Omega \times I \rightarrow \mathbb{R}$ be a potential satisfying the conditions of Lemma 13.2. Then, Theorems 2.19 - 2.23 hold for the random beta transformation $\left(T_{\omega}\right)_{\omega \in \Omega}$, provided

$$
\log s_{\omega, N_{*}} \in L_{m}^{1}(\Omega)
$$

where $N_{*}$ is defined in (4.6) and $s_{\omega, N_{*}}$ is length of the shortest branch of $z_{\omega}^{\left(N_{*}\right)}$.

The proof of this lemma relies on the following consequence of a result of Conze and Raugi [13, Lemma 3.5].

Lemma 13.9. Let $s_{\omega, N}$ be the length of the shortest branch of $z_{\omega}^{(N)}, M_{\omega, N}$ be the maximum of the covering time for the intervals of $Z_{\omega}^{(N)}$ under the sequence of maps $\left\{T_{\omega}^{n}\right\}_{n \in \mathbb{N}}$, and

$$
C=1+\frac{2}{\delta}=\sum_{r=0}^{\infty}\left(\frac{2}{2+\delta}\right)^{r}
$$

Then we have that

$$
M_{\omega, N} \leq\left\lceil\frac{-\log \frac{s_{\omega, N}}{C}}{\log \left(1+\frac{\delta}{2}\right)}\right\rceil+N .
$$

Proof. Let $0<\varepsilon<s_{\omega, N}$. The proof of [13, Lemma 3.5] shows that whenever $C\left(\frac{2}{2+\delta}\right)^{r} \leq \varepsilon$, or equivalently

$$
r \geq\left\lceil\frac{-\log \frac{\varepsilon}{C}}{\log \left(1+\frac{\delta}{2}\right)}\right\rceil,
$$

then $[0,1]$ is covered, up to a set of measure $\varepsilon$, by full branches for the maps $T_{\omega}^{N}, T_{\omega}^{N+1}, \ldots$, $T_{\omega}^{N+r-1}$. Note that this implies that if $Y \in \mathcal{Z}_{\omega}^{(N)}$ (and so $Y$ has measure greater than $\varepsilon$ ), and

$$
R \geq N+\left\lceil\frac{-\log \frac{\varepsilon}{C}}{\log \left(1+\frac{\delta}{2}\right)}\right\rceil,
$$

then $Y$ is a union of branches of $z_{\omega}^{(R)}$, and so it must contain a full branch for $T_{\omega}^{R}$. Thus, $M_{\omega, N} \leq R$. The conclusion follows from letting $\varepsilon \uparrow s_{\omega, N}$.

Proof of Lemma 13.8. The proof goes as in Section 13.1. Condition (GP) holds because the system is expanding on average. Hence, $(\mathrm{RC})$ follows from Lemma 13.9 because the partitions are finite (see Remark 2.8).

Let us assume $\varphi, \varphi_{-}, \varphi_{+} \in \mathbb{R}$ are as in Lemma 13.2. The summability conditions (Definition 2.2) are straightforward to check. Indeed, (SP2) is a hypothesis. (SP1) and (SP3) hold 
because $\varphi$ is bounded and the partitions are finite. When $g_{\omega}=\left|T_{\omega}^{\prime}\right|^{-t}$ for some $t \geq 0,(\mathrm{P} 1)$ holds with $\hat{\alpha}=0$ because $g_{\omega}^{(n)}$ is constant for each $\omega \in \Omega$. In general, (P2) holds with $\hat{\gamma}=1$ because $\mathcal{P}_{\omega, n}(0,1)$ coincides with the dynamical partition $z_{\omega}^{(n)}$.

Conditions (M1) and (M2) follow from $m$-continuity; see e.g. [21]. (M3) holds because the $\operatorname{map} x \mapsto \beta_{\omega} x+\alpha_{\omega}(\bmod 1)$ has at most $\tilde{b}_{\omega}:=\left\lceil\beta_{\omega}\right\rceil+1$ branches. Then, $e^{\varphi_{-}} \leq S_{\omega}^{(1)} \leq \tilde{b}_{\omega} e^{\varphi_{+}}$. Since $\beta_{\omega}$ is essentially bounded, say by $B$,

$$
\int_{\Omega}\left|\log S_{\omega}^{(1)}\right| d m \leq \max \left\{\left|\varphi_{-}\right|, \log (B+2)+\left|\varphi_{+}\right|\right\}<\infty .
$$

Notice that $\inf \mathcal{L}_{\omega} \mathbb{1}_{\omega} \geq n\left(T_{\omega}\right) e^{\varphi_{-}}$, where $n\left(T_{\omega}\right)=\min \left\{\# T_{\omega}^{-1}(x): x \in I\right\}$ is the minimum number of preimages of a point $x \in I$ under $T_{\omega}$. Since $T_{\omega}$ is a shifted $\beta$-transformation, $n\left(T_{\omega}\right)=\left\lfloor\beta_{\omega}\right\rfloor$. Thus, in view of Remark 2.16, with $N_{1}=N_{3}=1$, in order to show the potential is contracting (Definition 2.15), it is sufficient to have that

$$
\varphi_{+}<\varphi_{-}+\int_{\Omega} \log \left\lfloor\beta_{\omega}\right\rfloor d m
$$

as assumed. Note that this condition ensures that $\left\lfloor\beta_{\omega}\right\rfloor \geq 1$ for $m$ a.e. $\omega \in \Omega$, so $\beta_{\omega} \geq 1$ for $m$ a.e. $\omega \in \Omega$, and so $\log \inf \mathcal{L}_{\omega} \mathbb{1} \geq \varphi_{-}$. This yields (M4). Finally, we show conditions (M5') and (M6'), namely

$$
\log \left\|\mathcal{L}_{\omega}^{M_{\omega, N *}} \mathbb{1}\right\|_{\infty} \in L_{m}^{1}(\Omega), \quad \log \operatorname{ess} \inf g_{\omega}^{\left(M_{\omega, N_{*}}\right)} \in L_{m}^{1}(\Omega) .
$$

Note that

$$
M_{\omega, N_{*}} \varphi_{-} \leq \log \operatorname{essinf} g_{\omega}^{\left(M_{\omega, N_{*}}\right)} \leq \log \operatorname{ess} \sup g_{\omega}^{\left(M_{\omega, N_{*}}\right)} \leq M_{\omega, N_{*}} \varphi_{+} .
$$

Recalling that the map $x \mapsto \beta_{\omega} x+\alpha_{\omega}(\bmod 1)$ has at most $\tilde{b}_{\omega}:=\left\lceil\beta_{\omega}\right\rceil+1$ branches, we get

$$
\log \operatorname{ess} \inf g_{\omega}^{\left(M_{\omega, N_{*}}\right)} \leq \log \left\|\mathcal{L}_{\omega}^{M_{\omega, N_{*}}} \mathbb{1}\right\|_{\infty} \leq \log \operatorname{ess} \sup \left(\tilde{b}_{\omega}^{\left(M_{\omega, N_{*}}\right)} g_{\omega}^{\left(M_{\omega, N_{*}}\right)}\right),
$$

where $\tilde{b}_{\omega}^{(n)}=\prod_{j=0}^{n-1} \tilde{b}_{\sigma^{j} \omega}$. Therefore,

$$
M_{\omega, N_{*}} \varphi_{-} \leq \log \left\|\mathcal{L}_{\omega}^{M_{\omega, N_{*}}} \mathbb{1}\right\|_{\infty} \leq \log \left(\tilde{b}_{\omega}^{\left(M_{\omega, N_{*}}\right)}\right)+M_{\omega, N_{*}} \varphi_{+} .
$$

Lemma 13.9 shows that $\int_{\Omega} M_{\omega, N_{*}} d m<\infty$ whenever

$$
\int_{\Omega}\left|\log s_{\omega, N_{*}}\right| d m<\infty
$$

which is a hypothesis of the lemma. Recalling that $\beta_{\omega} \leq B$, we get

$$
\int \log \tilde{b}_{\omega}^{\left(M_{\omega, N_{*}}\right)} d m \leq \int M_{\omega, N_{*}} \log (B+2) d m<\infty .
$$

Hence, (13.12) is satisfied.

Remark 13.10. We could use similar techniques to show that Theorems 2.19-2.23 apply for maps with nonlinear branches. For example, let $S_{\omega}: I \rightarrow \mathbb{R}$ be a $C^{2}$ expanding map, with

$$
2+\delta<\operatorname{essinf}\left|S_{\omega}^{\prime}\right| \leq \operatorname{ess} \sup \left|S_{\omega}^{\prime}\right|<B
$$

for $m$ a.e. $\omega \in \Omega$, for some $0<\delta, B$, and let $\tilde{T}_{\omega}(x)=S_{\omega}(x)(\bmod 1)$. Each map $\tilde{T}_{\omega}(x)$ has at most two non-full branches (the leftmost and rightmost ones). For simplicity, assume $N_{*}=1$. Lemma 13.9 (with $N=1$ ) relies on [13, Lemma 3.5] for the case $n=1$ only, and an inspection of the proof shows that its conclusions remain valid for $\tilde{T}_{\omega}$ instead of $T_{\omega}$. Then, 
the proof of Lemma 13.8 remains applicable for $\tilde{T}_{\omega}$ with minor modifications, after replacing the hypothesis of Lemma 13.2 involving $E_{-}$with

$$
\varphi_{+}-\varphi_{-}<\int_{\Omega} \log \operatorname{ess} \inf \left|S_{\omega}^{\prime}\right| d m
$$

and ensuring hypothesis $(i i)$ of Lemma 13.2 is satisfied. These modifications essentially amount to changing $\left\lfloor\beta_{\omega}\right\rfloor$ and $\left\lceil\beta_{\omega}\right\rceil$ to ess inf $\left|S_{\omega}^{\prime}\right|$ and ess $\sup \left|S_{\omega}^{\prime}\right|$.

13.4. Random Maps With Infinitely Many Branches. In this section we consider compositions of the Gauss map $T_{0}$ and the Renyi map $T_{1}$, defined by

$$
T_{0}(x)=\left\{\begin{array}{lll}
0 & x=0, \\
\frac{1}{x} \quad(\bmod 1) & x \neq 0,
\end{array} \quad \text { and } \quad T_{1}(x)= \begin{cases}0 & x=1 \\
\frac{1}{1-x} \quad(\bmod 1) & x \neq 1\end{cases}\right.
$$

This class of random maps was investigated in [27] in connection with continued fraction expansions. Let $T: \Omega \rightarrow\{0,1\}$ be a measurable function. Let $T_{\omega}=T_{0}$ if $T(\omega)=0$ and $T_{\omega}=T_{1}$ otherwise. We denote by $\mathcal{L}_{i}$ the transfer operator associated to $T_{i}, i=0,1$. Since both $T_{0}$ and $T_{1}$ have only full branches, the system is covering, with $M_{\omega, n}=1$ for every $\omega \in \Omega, n \in \mathbb{N}$.

Lemma 13.11. Consider a potential of the form $\varphi(\omega, x)=-t \log \left|T_{\omega}^{\prime}(x)\right|$, for $0.5<t \leq$ 0.613. Then, the hypotheses of Theorems 2.19 - 2.23 hold.

Proof. We will use the shorthand notation $\varphi_{i}(x)=\varphi(\omega, x)$ whenever $T_{\omega}=T_{i}$, and similarly for $g_{i}(x)$. Note that since at the differentiability points, $\left|T_{0}^{\prime}(x)\right|=\frac{1}{x^{2}}$, then

$$
g_{0}(x)=x^{2 t}, \quad\left|\log g_{0}(x)\right|=\left|\varphi_{0}(x)\right|=2 t \log \frac{1}{x}
$$

and for each partition element $J_{j}=\left[\frac{1}{j+1}, \frac{1}{j}\right]$,

$$
\left|\log \underset{J_{j}}{\operatorname{essinf}} g_{\omega}\right|=2 t \log (j+1) \text {. }
$$

The analysis for $T_{1}$ is entirely analogous, since $T_{1}(x)=T_{0}(1-x)$.

Then,

(1) The summability conditions (Definition 2.2) are satisfied. (SP1) and (SP2) follow directly from the definition of $g_{\omega}$. (SP3) follows from the fact that

$$
\sum_{j=1}^{\infty} \sup _{J_{j}} g_{\omega}=\sum_{j=1}^{\infty} \frac{1}{j^{2 t}}<\infty
$$

The first part of (M3) holds because $\sup \varphi_{\omega}=0$, the second part follows from (SP3), because $S_{\omega}^{(1)}$ can only take two different values. Also, (M1) and (M2) hold because $T$ is measurable and of finite rank.

(2) Conditions (P1) and (P2), with $\hat{\alpha}=\hat{\gamma}=1$, are satisfied for $n=1$, with partitions $\mathcal{P}_{0,1}(1,1)=\mathcal{P}_{1,1}(1,1)$ given by $\mathcal{P}_{i}=\left\{Z_{1}^{i}, Z_{2}^{i}, \ldots, Z_{k}^{i}, Z_{\infty}^{i}\right\}$, for $i=0,1$, where $k=k(t)$ is chosen such that $\sum_{j=k+1}^{\infty} \frac{1}{j^{2 t}} \leq 1, Z_{j}^{0}=\left[\frac{1}{j+1}, \frac{1}{j}\right]$ for $1 \leq j \leq k, Z_{\infty}^{0}=\left[0, \frac{1}{k+1}\right]$, and $Z_{j}^{1}$ obtained from $Z_{j}^{0}$ by the transformation $x \mapsto 1-x$. Indeed $g_{\omega}$ is monotonic on $[0,1]$ and $\operatorname{var}_{[0,1]} g_{\omega}=1$, so $(\mathrm{P} 1)$ holds with $\hat{\alpha}=1$ regardless of the partition. The choice of $k$ precisely ensures condition (P2) holds with $\hat{\gamma}=1$ and $n=1$. 
(3) (CP1) holds, i.e. $\varphi$ is a contracting potential. In fact, we check that condition (4.6) holds with $N_{*}=1$, i.e. we have that

$$
\int_{\Omega} \log \frac{4\left\|g_{\omega}\right\|_{\infty}}{\inf \mathcal{L}_{\omega} \mathbb{1}} d m<0
$$

Indeed, $\left\|g_{0}(x)\right\|_{\infty}=1$ and $\mathcal{L}_{0} \mathbb{1}(x)=\sum_{j=1}^{\infty} \frac{1}{(j+x)^{2 t}}$, so

$$
\frac{4\left\|g_{0}\right\|_{\infty}}{\inf \mathcal{L}_{0} \mathbb{1}}=\frac{4}{\sum_{j=2}^{\infty} \frac{1}{j^{2 t}}}=\frac{4}{\zeta(2 t)-1}<1
$$

because $\zeta(1.226)>5$, where $\zeta(x)$ is the Riemann zeta function. For $T_{\omega}=T_{1}$, $g_{1}(x)=g_{0}(1-x)$ so the bound is the same.

(4) In light of Remark 4.8 we check that conditions (M5'), (M6') hold for $N_{*}=1$. For each $\omega \in \Omega$, the elements of $\mathcal{P}_{\omega}$ consist of (unions of) full branches for $T_{\omega}$. Hence, $N_{\omega, 1}(P)=1$ (coming from $(2.10)$ ) and $M_{\omega}(J(P))=1$ (coming from $(\mathrm{RC})$ ) for every $P \in \mathcal{P}_{\omega}$. In particular, for every $\omega \in \Omega$,

$$
\zeta(2 t)-1 \leq \inf \mathcal{L}_{\omega} \mathbb{1} \leq\left\|\mathcal{L}_{\omega} \mathbb{1}\right\|_{\infty} \leq \zeta(2 t)<\infty
$$

for $t>\frac{1}{2}$, so the integrability condition $\left(\mathrm{M}^{\prime}\right)$ is satisfied. Also, by choosing $J(P)$ to be the largest full branch in $P$, we get that

$$
\log \underset{J(P)}{\operatorname{essinf}} g_{\omega} \geq-2 t \log (k+2)
$$

for each $\omega \in \Omega$. Hence the integrability condition (M5') is satisfied.

Remark 13.12. Note that we have only chosen $t \in(0.5,0.613]$ to ensure that we have $N_{*}=1$. One could treat larger values of $t$, in particular $t \geq 1$, by considering $N_{*}>1$.

13.5. Random Non-Uniformly Expanding Maps. In this section we consider random compositions of a $\beta$-transformation $T_{0}$ and the Pomeau-Manneville intermittent map $T_{1}$, defined by

$$
T_{0}(x)=\beta x \quad \bmod 1 \quad \text { and } \quad T_{1}(x)= \begin{cases}x+2^{a} x^{1+a} & \text { for } x \in[0,1 / 2] \\ 2 x-1 & \text { for } x \in(1 / 2,1]\end{cases}
$$

where $a>0$ and $\beta \geq 5$. The map $T_{1}$, in this form, was first introduced in [34], but dates back to the works of $[35,43]$. Sequential and random compositions of $T_{1}$, and maps similar to $T_{1}$, taken with varying values for $a$, have been investigated previously, see e.g. [1, 41].

Let $T: \Omega \rightarrow\{0,1\}$ be a measurable function. Let $T_{\omega}=T_{0}$ if $T(\omega)=0$ and $T_{\omega}=T_{1}$ otherwise. We denote by $\mathcal{L}_{i}$ the transfer operator associated to $T_{i}, i=0,1$.

Lemma 13.13. Consider a potential of the form $\varphi(\omega, x)=-t \log \left|T_{\omega}^{\prime}(x)\right|$, for $t \geq 0$. If

$$
0<p=m\left(T^{-1}(1)\right)<\frac{\log \frac{\lfloor\beta\rfloor}{4}}{\log \frac{\lfloor\beta\rfloor(2 a+4)^{t}}{2^{t}+(a+2)^{t}}}
$$

then the hypotheses of Theorems 2.19 - 2.23 hold. 
Proof. We begin by noting that our random covering assumption (RC) follows from the Birkhoff Ergodic Theorem as for $n$ sufficiently large we will see the map $T_{0}$ with frequency $(1-p) n$, and thus any small interval will eventually cover. As each map has only finitely many branches (SP1)-(SP3) are satisfied. Now, we note that

$$
\inf g_{0}=\left\|g_{0}\right\|_{\infty}=\frac{1}{\beta^{t}}, \quad \inf g_{1}=\frac{1}{(a+2)^{t}}, \quad \text { and } \quad\left\|g_{1}\right\|_{\infty}=1 \text {. }
$$

It then follows that

$$
\frac{\lfloor\beta\rfloor}{\beta^{t}} \leq \inf \mathcal{L}_{0} \mathbb{1} \leq\left\|\mathcal{L}_{0} \mathbb{1}\right\|_{\infty} \leq \frac{\lceil\beta\rceil}{\beta^{t}} \leq\lceil\beta\rceil
$$

and

$$
\frac{2^{t}+(a+2)^{t}}{(2 a+4)^{t}}=\frac{1}{(a+2)^{t}}+\frac{1}{2^{t}} \leq \inf \mathcal{L}_{1} \mathbb{1} \leq\left\|\mathcal{L}_{1} \mathbb{1}\right\|_{\infty} \leq 1+\frac{1}{2^{t}} \leq 2 .
$$

Thus conditions (M1)-(M4) are satisfied. Concerning the variation, we note that $\operatorname{var}_{Z}\left(g_{0}\right)=$ 0 for each $Z \in Z_{0}^{(1)}, \operatorname{var}_{[0,1 / 2]}\left(g_{1}\right)=1, \operatorname{var}_{[1 / 2,1]}\left(g_{1}\right)=0$. Thus, in view of Remark 2.9 , the partition $\mathcal{P}_{i, 1}(1,1)=Z_{i}^{(1)}$ satisfies $(\mathrm{P} 1)$ and $(\mathrm{P} 2)$ with $\hat{\alpha}=\hat{\gamma}=1$ for each $i \in\{0,1\}$.

We claim now that we can take the number $N_{*}$, coming from (4.6), to be 1 . Indeed, since

$$
\begin{aligned}
\int_{\Omega} \log \frac{4\left\|g_{\omega}\right\|_{\infty}}{\inf \mathcal{L}_{\omega} \mathbb{1}_{\omega}} d m(\omega) & =(1-p) \log \frac{4\left\|g_{0}\right\|_{\infty}}{\inf \mathcal{L}_{0} \mathbb{1}}+p \log \frac{4\left\|g_{1}\right\|_{\infty}}{\inf \mathcal{L}_{1} \mathbb{1}} \\
& \leq(1-p) \log \frac{4 / \beta^{t}}{\lfloor\beta\rfloor / \beta^{t}}+p \log \frac{4}{\left(2^{t}+(a+2)^{t}\right) /(2 a+4)^{t}} \\
& =(1-p) \log \frac{4}{\lfloor\beta\rfloor}+p \log \frac{4(2 a+4)^{t}}{2^{t}+(a+2)^{t}},
\end{aligned}
$$

(4.6) holds whenever

$$
p<\frac{\log \frac{\lfloor\beta\rfloor}{4}}{\log \frac{\lfloor\beta\rfloor(2 a+4)^{t}}{2^{t}+(a+2)^{t}} .}
$$

Thus, as we have chosen $p>0$ satisfying (13.16), we see that

$$
\int_{\Omega} \log \frac{4\left\|g_{\omega}\right\|_{\infty}}{\inf \mathcal{L}_{\omega} \mathbb{1}_{\omega}} d m(\omega)<0
$$

In light of Remark 4.8, we now check conditions (M5') and (M6'). To that end, we note that (M5') holds since $\inf _{Z} g_{0}=1 / \beta^{t}$ for each $Z \in z_{0}^{(1)}, \inf _{[0,1 / 2]} g_{1}=1 /(a+2)^{t}$, and $\inf _{[1 / 2,1]} g_{1}=1 / 2^{t}$, and (M6') follows from (13.13) and (13.14). Finally, we see that (CP1) and (CP2) hold in light of (13.17) and Remark 2.16.

Remark 13.14. Notice that our choice of $\beta \geq 5$ was instrumental in obtaining $N_{*}=1$. Using similar arguments one could yield the conclusions of Lemma 13.13 with any $\beta \geq 2$ and $N_{*}=3$ for $p>0$ sufficiently small.

Furthermore, it is interesting to point out that our results hold for any positive value of the parameter $a$ defining the intermittent map $T_{1}$. This is in contrast to the deterministic setting (of the map $T_{1}$ together with potential $\varphi=-\log \left|T_{1}^{\prime}\right|$ ), where the density with respect to the conformal measure, which is the Lebesgue measure, for the map $T_{1}$ is Lebesgue summable only for $a<1$. 
Also note that the upper bound on $p$ tends to zero as $t$ tends to infinity.

Remark 13.15. Here we have chosen, for the ease of computation and exposition, to present Example 13.5 as the random iteration of two maps each with positive probability, however, assuming the $m$-continuity conditions of Lemma 13.2, one could easily apply similar arguments to the setting where one considers maps of the form

$$
T_{\omega}(x)= \begin{cases}\beta_{\omega} x \bmod 1 & \text { if } \omega \in \Omega_{0} \\ \begin{cases}x+2^{a_{\omega}} x^{1+a_{\omega}} & \text { for } x \in[0,1 / 2] \\ 2 x-1 & \text { for } x \in(1 / 2,1]\end{cases} & \text { if } \omega \in \Omega_{1}\end{cases}
$$

where $\Omega=\Omega_{0} \cup \Omega_{1}$ is infinite with $\Omega_{0} \cap \Omega_{1}=\emptyset$ such that $m\left(\Omega_{0}\right), m\left(\Omega_{1}\right)>0$, and where the maps $\omega \mapsto a_{\omega} \in\left(0, a^{*}\right]$, for some $a^{*}>0, \omega \mapsto \beta_{\omega} \in[5, \infty)$.

Note that the "intermittency" of the map $T_{1}$ in the previous lemma is a bit misleading. We chose $p$ so that $T_{1}$ was applied sufficiently infrequently to achieve a map cocycle that was expanding on average. This same technique allows us to deal with maps with contracting branches $^{13}$. Indeed, if we consider the maps

$$
T_{0}(x)=\beta x \quad \bmod 1 \quad \text { and } \quad T_{1}(x)= \begin{cases}a x & \text { for } x \in[0,1 / 2] \\ (2-a) x-(1-a) & \text { for } x \in(1 / 2,1]\end{cases}
$$

with $0<a<1$ and $\beta \geq 5^{14}$, in the same setup as the previous example, then using similar reasoning we arrive at the following lemma.

Lemma 13.16. Consider a potential of the form $\varphi(\omega, x)=-t \log \left|T_{\omega}^{\prime}(x)\right|$, for $t \geq 0$. If

$$
0<p=m\left(T^{-1}(1)\right)<\frac{\log \frac{\lfloor\beta\rfloor}{4}}{\log \frac{\lfloor\beta\rfloor(2-a)^{t}}{a^{t}}},
$$

then the hypotheses of Theorems 2.19 - 2.23 hold.

Remark 13.17. As with Lemma 13.13, we note again that $p$ must go to zero as $t$ tends toward infinity.

13.6. Random Lasota-Yorke maps. In this final example we discuss the very broad class of random piecewise-monotonic maps, where each fiber map $T_{\omega}$ is a finite-branched map which takes the interval onto itself. We consider a general potential $\varphi \in \mathrm{BV}_{\Omega}(I)$. Recall that $\# Z_{\omega}^{(1)}$ denotes the number of branches of monotonicity of $T_{\omega}$, and for each $n \in \mathbb{N}$ define $\mathcal{J}_{\omega}^{(n)}:=\min _{y \in[0,1]} \#\left\{T_{\omega}^{-n}(y)\right\}$.

Lemma 13.18. Under the m-continuity conditions of Lemma 13.2, we assume the integrability conditions $\log \mathfrak{J}_{\omega}^{(1)}, \log \# \mathcal{Z}_{\omega}^{(1)}, \inf \varphi_{\omega}, \sup \varphi_{\omega} \in L_{m}^{1}(\Omega)$, and that

$$
\int_{\Omega}\left(\sup \varphi_{\omega}-\inf \varphi_{\omega}\right) d m(\omega)<\int_{\Omega} \log \mathcal{J}_{\omega}^{(1)} d m(\omega) .
$$

Then conditions (M1)-(M4) hold and the potential $\varphi$ is summable and contracting, i.e. conditions (SP1)-(SP3) and (CP1)-(CP2) hold.

\footnotetext{
${ }^{13}$ The maps are still required to be onto, however some branches may be contracting.

${ }^{14}$ The same argument given in Remark 13.14 allows us to replace $\beta \geq 5$ with $\beta \geq 2$ at the expense of taking $N_{*}=3$.
} 
Proof. In light of Remark 2.3 we see that (SP1)-(SP3) hold since $\# \mathbb{Z}_{\omega}^{(1)}<\infty$ and $\varphi \in$ $\mathrm{BV}_{\Omega}(I)$. Conditions (M1) and (M2) hold by $m$-continuity, see [21]. For conditions (M3)(M4), we note that because

$$
\log \mathcal{J}_{\omega}^{(1)}+\inf \varphi_{\omega} \leq \log \inf \mathcal{L}_{\omega} \mathbb{1} \leq \log S_{\omega}^{(1)} \leq \log \# \mathcal{Z}_{\omega}^{(1)}+\sup \varphi_{\omega},
$$

these conditions hold by the integrability hypotheses on the terms in the above inequalities. Using (13.18), we have

$$
\begin{aligned}
\int_{\Omega} \log \left\|g_{\omega}\right\|_{\infty} d m(\omega) & =\int_{\Omega} \sup \varphi_{\omega} d m(\omega) \\
& <\int_{\Omega} \log \mathcal{J}_{\omega}^{(1)} d m(\omega)+\int_{\Omega} \inf \varphi_{\omega} d m(\omega) \leq \int_{\Omega} \log \inf \mathcal{L}_{\omega} \mathbb{1} d m(\omega),
\end{aligned}
$$

and thus, applying (2.13) with $N_{1}=N_{2}=1$, we see that $\varphi$ is a contracting potential.

Remark 13.19. Using similar arguments, we could replace (13.18) in Lemma 13.18 with the following: there exists $N \in \mathbb{N}$ such that $\log \mathcal{J}_{\omega}^{(N)} \in L_{m}^{1}(\Omega)$ and

$$
\int_{\Omega} \sup S_{N, T}\left(\varphi_{\omega}\right)-\inf S_{N, T}\left(\varphi_{\omega}\right) d m(\omega)<\int_{\Omega} \log \mathcal{J}_{\omega}^{(N)} d m(\omega) .
$$

Corollary 13.20. Assuming the hypotheses of Lemma 13.18, if, in addition, we assume the random covering condition ( $\mathrm{RC}$ ) and the general integrable covering conditions (M5), (M6), then the hypotheses of Theorems 2.19 - 2.23 hold.

We now present a final example of the class of random Lasota-Yorke maps focusing on geometric potentials, i.e. potentials of the form $\varphi_{\omega}=-t \log \left|T_{\omega}^{\prime}\right|$ for $t \geq 0$. We will require the following strengthening of our random covering assumption (RC) which has appeared before in [2] and [24].

Definition 13.21. We say that the family $\left(T_{\omega}\right)_{\omega \in \Omega}$ satisfies strong random covering if there exists $M(n) \in \mathbb{N}$ such that for any $\omega \in \Omega$ and any $Z \in \mathcal{Z}_{\omega}^{(n)}$ we have that $T_{\omega}^{M(n)}(Z)=I$.

Remark 13.22. Note that this strong random covering condition is satisfied by the maps considered in Section 13.2.

Lemma 13.23. Let $\varphi_{\omega}=-t \log \left|T_{\omega}^{\prime}\right|$ for $t \geq 0$, and assume the $m$-continuity conditions of Lemma 13.2. We further suppose the system satisfies strong random covering as well as the following:

(1) for each $\omega \in \Omega, Z \in Z_{\omega}^{*}$, and $x \in Z$

(a) $\left.T_{\omega}\right|_{Z} \in C^{2}$,

(b) there exists $K \geq 1$ such that

$$
\frac{\left|T_{\omega}^{\prime \prime}(x)\right|}{\left|T_{\omega}^{\prime}(x)\right|} \leq K
$$

(2) there exists $n_{0} \in \mathbb{N}$ and $1<\lambda \leq \Lambda<\infty$ such that

(a) $\left|T_{\omega}^{\prime}\right| \leq \Lambda$ for $m$-a.e. $\omega \in \Omega$,

(b) $\left|\left(T_{\omega}^{n_{0}}\right)^{\prime}\right| \geq \lambda^{n_{0}}$ for $m$-a.e. $\omega \in \Omega$,

(c) $\frac{1}{n_{0}} \int_{\Omega} \log \mathcal{J}_{\omega}^{\left(n_{0}\right)} d m(\omega)>t \log \frac{\Lambda}{\lambda}$, 
(3) for each $n \in \mathbb{N}$ there exists

$$
\delta_{n}:=\inf _{\omega \in \Omega} \min _{Z \in z_{\omega}^{(n)}} \operatorname{diam}(Z)>0 .
$$

Then the hypotheses of Theorems 2.19 - 2.23 hold.

Proof. First we note that hypothesis (2) implies that we have

$$
\Lambda^{-k n_{0} t} \leq g_{\omega}^{\left(k n_{0}\right)} \leq \lambda^{-k n_{0} t}
$$

for each $\omega \in \Omega$ and $k \in \mathbb{N}$, and therefore $\inf S_{k n_{0}, T}\left(\varphi_{\omega}\right)$, sup $S_{k n_{0}, T}\left(\varphi_{\omega}\right) \in L_{m}^{1}(\Omega)$ for each $k \in \mathbb{N}$. Furthermore, (13.19), together with hypothesis (2), gives that

$$
\frac{1}{n_{0}} \int_{\Omega} \sup S_{n_{0}, T}\left(\varphi_{\omega}\right)-\inf S_{n_{0}, T}\left(\varphi_{\omega}\right) d m(\omega) \leq t \log \frac{\Lambda}{\lambda}<\frac{1}{n_{0}} \int_{\Omega} \log \mathfrak{J}_{\omega}^{\left(n_{0}\right)} d m(\omega) .
$$

To see that the last of the hypotheses of Lemma 13.18 is satisfied, we note that hypothesis (3) implies that there exists $D \in \mathbb{N}$ such that

$$
\# \mathcal{Z}_{\omega}^{(1)} \leq D
$$

for each $\omega \in \Omega$, which of course implies that $\log \# Z_{\omega}^{(1)} \in L_{m}^{1}(\Omega)$. Thus, Lemma 13.18 and Remark 13.19 imply that $\varphi$ is contracting and summable and that conditions (M1)(M4) hold. Thus, given our strong random covering assumption, we have only to find an appropriate partition $\mathcal{P}_{\omega, n}(\hat{\alpha}, \hat{\gamma})$ (satisfying (P1) and (P2)) for which we can check conditions (M5') and (M6') for $N_{*}$.

To that end, using hypotheses (1) and (2), we note that for any $\omega \in \Omega, k \in \mathbb{N}, Z \in Z_{\omega}^{\left(k n_{0}\right)}$, and any interval $J \subseteq Z$ we have that

$$
\begin{aligned}
\operatorname{var}_{J}\left(g_{\omega}^{\left(k n_{0}\right)}\right) & \leq 2\left\|g_{\omega}^{\left(k n_{0}\right)}\right\|_{\infty}+\int_{J}\left|\left(g_{\omega}^{\left(k n_{0}\right)}(x)\right)^{\prime}\right| d x \\
& \leq 2\left\|g_{\omega}^{\left(k n_{0}\right)}\right\|_{\infty}+t \int_{J} \sum_{j=0}^{k n_{0}-1}\left(\frac{\left|T_{\sigma^{j}(\omega)}^{\prime \prime}\left(T_{\omega}^{j}(x)\right)\right|}{\left|T_{\sigma^{j}(\omega)}^{\prime}\left(T_{\omega}^{j}(x)\right)\right|} \cdot \frac{\prod_{i=0}^{j-1}\left|T_{\sigma^{i}(\omega)}^{\prime}\left(T_{\omega}^{i}(x)\right)\right|}{\prod_{i=0}^{k n_{0}-1}\left|T_{\sigma^{i}(\omega)}^{\prime}\left(T_{\omega}^{i}(x)\right)\right|^{t}}\right) d x \\
& \leq 2\left\|g_{\omega}^{\left(k n_{0}\right)}\right\|_{\infty}+t K \cdot \frac{\sum_{j=0}^{k n_{0}-1} \Lambda^{j}}{\lambda^{n t}} \cdot \operatorname{diam}(J) \\
& \leq 2\left\|g_{\omega}^{\left(k n_{0}\right)}\right\|_{\infty}+\frac{t K}{\Lambda-1} \cdot\left(\frac{\Lambda}{\lambda^{t}}\right)^{k n_{0}} \cdot \operatorname{diam}(J) .
\end{aligned}
$$

Now we for each $Z \in Z_{\omega}^{(n)}$, we subdivide $Z$ into

$$
v_{n}:=\left\lceil\frac{\Lambda}{\lambda^{t}}\right\rceil^{n} \cdot\left\lceil\frac{\Lambda^{t}}{\lambda}\right\rceil^{n}
$$

many pieces $P_{Z, 1}, \cdots, P_{Z, v_{n}}$ of equal length. Since hypotheses (2)(b) and (3) imply that for each $\omega \in \Omega, k \in \mathbb{N}$, and $Z \in Z_{\omega}^{\left(k n n_{0}\right)}$ we have that

$$
\delta_{k n_{0}} \leq \operatorname{diam}(Z) \leq \lambda^{-k n_{0}},
$$

then for any $P_{Z, j} \subseteq Z\left(1 \leq j \leq v_{k n_{0}}\right)$ we have

$$
\delta_{k n_{0}} v_{k n_{0}}^{-1} \leq \operatorname{diam}\left(P_{Z, j}\right)=\left\lceil\frac{\Lambda}{\lambda^{t}}\right\rceil^{-k n_{0}} \cdot\left\lceil\frac{\Lambda^{t}}{\lambda}\right\rceil^{-k n_{0}} \cdot \operatorname{diam}(Z) \leq\left\lceil\frac{\Lambda}{\lambda^{t}}\right\rceil^{-k n_{0}} \cdot \frac{1}{\lambda^{k n_{0}}\left(\frac{\Lambda^{t}}{\lambda}\right)^{k n_{0}}}
$$




$$
\leq\left\lceil\frac{\Lambda}{\lambda^{t}}\right]^{-k n_{0}} \Lambda^{-k n_{0} t} \leq\left\lceil\frac{\Lambda}{\lambda^{t}}\right]^{-k n_{0}}\left\|g_{\omega}^{\left(k n_{0}\right)}\right\|_{\infty} .
$$

Thus, inserting (13.21) into (13.20) gives

$$
\begin{aligned}
\operatorname{var}_{P_{Z, j}}\left(g_{\omega}^{\left(k n_{0}\right)}\right) & \leq 2\left\|g_{\omega}^{\left(k n_{0}\right)}\right\|_{\infty}+\frac{t K}{\Lambda-1} \cdot\left(\frac{\Lambda}{\lambda^{t}}\right)^{k n_{0}} \cdot \operatorname{diam}\left(P_{Z, j}\right) \\
& \leq 2\left\|g_{\omega}^{\left(k n_{0}\right)}\right\|_{\infty}+\frac{t K}{\Lambda-1} \cdot\left(\frac{\Lambda}{\lambda^{t}}\right)^{k n_{0}} \cdot\left\lceil\frac{\Lambda}{\lambda^{t}}\right]^{-k n_{0}}\left\|g_{\omega}^{\left(k n_{0}\right)}\right\|_{\infty} \\
& \leq\left(2+\frac{t K}{\Lambda-1}\right)\left\|g_{\omega}^{\left(k n_{0}\right)}\right\|_{\infty} .
\end{aligned}
$$

In light of (13.22) and the fact that $P_{Z, j} \subseteq Z$ for each $1 \leq j \leq v_{k n_{0}}$, we note that conditions (P1) and (P2) are satisfied for the partition

$$
\mathcal{P}_{\omega, k n_{0}}=\mathcal{P}_{\omega, k n_{0}}\left(2+\frac{t K}{\Lambda-1}, 1\right):=\left\{P_{Z, j}: 1 \leq j \leq v_{k n_{0}}, Z \in Z_{\omega}^{\left(k n_{0}\right)}\right\} .
$$

Let $k_{*}$ be the minimum integer such that

$$
\int_{\Omega} \log \frac{\left(5+\frac{t K}{\Lambda-1}\right)\left\|g_{\omega}^{\left(k_{*} n_{0}\right)}\right\|_{\infty}}{\inf \mathcal{L}_{\omega}^{k_{*} n_{0}} \mathbb{1}} d m(\omega)<0
$$

and let $N_{*}=k_{*} n_{0}$. Now let $k_{1} \in \mathbb{N}$ be the first integer such that for each $P \in \mathcal{P}_{\omega, N_{*}}$ we have

$$
\lambda^{-k_{1} n_{0}} \leq \frac{\delta_{N_{*}}}{2 v_{N_{*}}} \leq \frac{1}{2} \operatorname{diam}(P) .
$$

Thus for each $P \in \mathcal{P}_{\omega, N_{*}}$ there exists $J \in \mathcal{Z}_{\omega}^{\left(k_{1} n_{0}\right)}$ such that $J \subseteq P$, and by strong random covering we have that $T_{\omega}^{M\left(k_{1} n_{0}\right)}(J)=I$. Finally, we see that (M5') and (M6') are satisfied since for each $\omega \in \Omega$, each $P \in \mathcal{P}_{\omega, N_{*}}$, and each $J \subseteq P$ we have

$$
\inf _{J} g_{\omega}^{\left(M\left(k_{1} n_{0}\right)\right)} \geq \Lambda^{-M\left(k_{1} n_{0}\right) t}
$$

and

$$
\left\|\mathcal{L}_{\omega}^{M\left(k_{1} n_{0}\right)} \mathbb{1}\right\|_{\infty} \leq\left\|g_{\omega}^{\left(M\left(k_{1} n_{0}\right)\right)}\right\|_{\infty} D^{M\left(k_{1} n_{0}\right)} \leq \lambda^{-M\left(k_{1} n_{0}\right) t} D^{M\left(k_{1} n_{0}\right)} .
$$

Remark 13.24. Note that if the set $\left\{T_{\omega}: \omega \in \Omega\right\}$ is finite, i.e. we have only finitely many maps, then hypothesis (3) of Lemma 13.23 always holds.

\section{ACKNOWLEDGMENTS}

J.A., G.F., and C.G.-T. thank the Centro de Giorgi in Pisa and CIRM in Luminy for their support and hospitality.

J.A. is supported by an ARC Discovery project and thanks the School of Mathematics and Physics at the University of Queensland for their hospitality.

G.F., C.G.-T., and S.V. are partially supported by an ARC Discovery Project.

S.V. thanks the Laboratoire International Associé LIA LYSM, the INdAM (Italy), the UMICNRS 3483, Laboratoire Fibonacci (Pisa) where this work has been completed under a CNRS delegation and the Centro de Giorgi in Pisa for various supports. 


\section{REFERENCES}

[1] Romain Aimino, Huyi Hu, Matthew Nicol, Andrei Török, and Sandro Vaienti. Polynomial loss of memory for maps of the interval with a neutral fixed point. Discrete 8 Continuous Dynamical Systems - A, 35(3):793, 2015. 69

[2] Romain Aimino and Jérôme Rousseau. Concentration inequalities for sequential dynamical systems of the unit interval. Ergodic Theory and Dynamical Systems, 36(8):2384-2407, December 2016. Publisher: Cambridge University Press. 72

[3] Alexander Arbieto and Carlos Matheus. Fast decay of correlations of equilibrium states of open classes of non-uniformly expanding maps and potentials. arXiv:math/0603629, March 2006. arXiv: math/0603629. 2

[4] Jason Atnip and Mariusz Urbański. Critically finite random maps of an interval. Discrete 83 Continuous Dynamical Systems - A, 40(8):4839, 2020. 3

[5] Garrett Birkhoff. Lattice Theory. American Mathematical Society, New York, 1940. 24, 25

[6] Thomas Bogenschütz. Equilibrium states for random dynamical systems. PhD Thesis, Institut für Dynamische Systeme, Universität Bremen, 1993. 14

[7] Thomas Bogenschütz. Entropy, pressure, and a variational principle for random dynamical systems. Random Comput. Dynam, 1(1):99-116, 1992. 2

[8] Thomas Bogenschütz and Volker Matthias Gundlach. Ruelle's Transfer Operator for Random Subshifts of Finite Type. Ergod. Th. \& Dynam. Sys, 15:413-447, 1995. 2

[9] Rufus Bowen. Equilibrium states and the ergodic theory of Anosov diffeomorphisms. Lecture Notes in Mathematics, Vol. 470. Springer-Verlag, Berlin-New York, 1975. 2

[10] Jérôme Buzzi. Exponential Decay of Correlations for Random Lasota-Yorke Maps. Communications in Mathematical Physics, 208(1):25-54, 1999. 3, 9, 18, 21, 27, 30, 34, 36

[11] Jérôme Buzzi and Omri Sarig. Uniqueness of equilibrium measures for countable Markov shifts and multidimensional piecewise expanding maps. Ergodic Theory and Dynamical Systems, 23(5):1383-1400, 2003. 2

[12] Armando Castro and Paulo Varandas. Equilibrium states for non-uniformly expanding maps: decay of correlations and strong stability. Annales de l'Institut Henri Poincare (C) Non Linear Analysis, 30(2):225-249, 2013. 2

[13] Jean-Pierre Conze and Albert Raugi. Limit theorems for sequential expanding dynamical systems on [0,1]. In Ergodic theory and related fields, volume 430 of Contemp. Math., pages 89-121. Amer. Math. Soc., Providence, RI, 2007. 61, 66, 67

[14] Hans Crauel. Random probability measures on Polish spaces, volume 11 of Stochastics Monographs. Taylor \& Francis, London, 2002. 8

[15] Manfred Denker, Gerhard Keller, and Mariusz Urbański. On the uniqueness of equilibrium states for piecewise monotone mappings. Studia Mathematica, 97(1):27-36, 1990. 2, 12

[16] Manfred Denker, Yuri Kifer, and Manuel Stadlbauer. Thermodynamic formalism for random countable Markov shifts. Discrete and Continuous Dynamical Systems. Series A, 22(1-2):131-164, 2008. 2

[17] Manfred Denker and Mariusz Urbański. On the existence of conformal measures. Transactions of the American Mathematical Society, 328(2):563-587, 1991. 17

[18] Davor Dragicevic, Gary Froyland, Cecilia Gonzalez-Tokman, and Sandro Vaienti. A spectral approach for quenched limit theorems for random expanding dynamical systems. Communications in Mathematical Physics, January 2018. arXiv: 1705.02130. 55

[19] D Dragičević, G Froyland, C González-Tokman, and S Vaienti. Almost sure invariance principle for random piecewise expanding maps. Nonlinearity, 31(5):2252-2280, May 2018. 55

[20] P. Ferrero and B. Schmitt. Produits aléatoires d'opérateurs matrices de transfert. Probability Theory and Related Fields, 79(2):227-248, 1988. 24

[21] G. Froyland, S. Lloyd, and A. Quas. A semi-invertible Oseledets theorem with applications to transfer operator cocycles. Discrete Contin. Dyn. Syst., 33(9):3835-3860, 2013. 62, 65, 67, 72

[22] Cecilia González-Tokman and Anthony Quas. Stability and Collapse of the Lyapunov spectrum for Perron-Frobenius Operator cocycles. arXiv:1806.08873 [math], November 2018. arXiv: 1806.08873. 30

[23] Volker M Gundlach. Thermodynamic formalism for random subshifts of finite type. 1996. 2 
[24] Nicolai Haydn, Matthew Nicol, Andrew Török, and Sandro Vaienti. Almost sure invariance principle for sequential and non-stationary dynamical systems. Transactions of the American Mathematical Society, 369(8):5293-5316, January 2017. 72

[25] Franz Hofbauer and Gerhard Keller. Equilibrium states for piecewise monotonic transformations. Ergodic Theory and Dynamical Systems, 2(1):23-43, March 1982. 3, 14

[26] Joseph Horan. Asymptotics for the second-largest Lyapunov exponent for some Perron-Frobenius operator cocycles. December 2019. arXiv: 1910.12112. 3

[27] Charlene Kalle, Tom Kempton, and Evgeny Verbitskiy. The random continued fraction transformation. Nonlinearity, 30(3):1182-1203, 2017. 68

[28] K. Khanin and Y. Kifer. Thermodynamic formalism for random transformations and statistical mechanics. In Sinai's Moscow Seminar on Dynamical Systems, volume 171 of Amer. Math. Soc. Transl. Ser. 2, pages 107-140. Amer. Math. Soc., Providence, RI, 1996. 2

[29] Yuri Kifer. Equilibrium states for random expanding transformations. Random \& Computational Dynamics, 1(1):1-31, 1992. 2

[30] François Ledrappier and Peter Walters. A relativised variational principle for continuous transformations. Journal of the London Mathematical Society, 2(3):568-576, 1977. 2

[31] Carlangelo Liverani. Decay of Correlations. Annals of Mathematics, 142(2):239-301, 1995. 24

[32] Carlangelo Liverani. Decay of correlations for piecewise expanding maps. Journal of Statistical Physics, 78(3):1111-1129, February 1995. 26

[33] Carlangelo Liverani, Benoit Saussol, and Sandro Vaienti. Conformal measure and decay of correlation for covering weighted systems. Ergodic Theory and Dynamical Systems, 18(6):1399-1420, December 1998. 2, 3, 4, 9, 10, 11, 12, 13, 18, 24, 25, 26, 36, 56

[34] Carlangelo Liverani, Benoît Saussol, and Sandro Vaienti. A probabilistic approach to intermittency. Ergodic Theory and Dynamical Systems, 19(3):671-685, June 1999. 69

[35] P. Manneville. Intermittency, self-similarity and 1/f spectrum in dissipative dynamical systems. Journal de Physique, 41(11):1235-1243, 1980. 69

[36] R. Daniel Mauldin and Mariusz Urbański. Graph directed Markov systems geometry and dynamics of limit sets. Cambridge University Press, Cambridge, 2003. 2

[37] Dieter H. Mayer. Approach to equilibrium for locally expanding maps in $\mathbf{R}^{k}$. Communications in Mathematical Physics, 95(1):1-15, March 1984. 24

[38] Volker Mayer and Mariusz Urbański. Countable alphabet random subhifts of finite type with weakly positive transfer operator. Journal of Statistical Physics, 160(5):1405-1431, 2015. 2

[39] Volker Mayer, Mariusz Urbański, and Bartlomiej Skorulski. Distance Expanding Random Mappings, Thermodynamical Formalism, Gibbs Measures and Fractal Geometry, volume 2036 of Lecture Notes in Mathematics. Springer Berlin Heidelberg, Berlin, Heidelberg, 2011. 2, 3, 14, 56

[40] Volker Mayer and Mariusz Urbański. Random dynamics of transcendental functions. Journal d'Analyse Mathématique, 134(1):201-235, February 2018. 55

[41] Matthew Nicol, Andrew Török, and Sandro Vaienti. Central limit theorems for sequential and random intermittent dynamical systems. Ergodic Theory and Dynamical Systems, 38(3):1127-1153, May 2018. 69

[42] W. Parry. On the $\beta$-expansions of real numbers. Acta Mathematica Academiae Scientiarum Hungarica, 11(3):401-416, September 1960. 61

[43] Yves Pomeau and Paul Manneville. Intermittent transition to turbulence in dissipative dynamical systems. Communications in Mathematical Physics, 74(2):189-197, June 1980. 69

[44] Feliks Przytycki and Mariusz Urbański. Conformal fractals: ergodic theory methods, volume 371. Cambridge University Press, 2010. 2

[45] Mario Roy and Mariusz Urbański. Random graph directed Markov systems. Discrete Contin. Dyn. Syst, 30(1):261-298, 2011. 2

[46] David Ruelle. Thermodynamic Formalism, volume 5 of Encyclopedia of Mathematics and its Applications. Addison-Wesley Publishing Co., Reading, Mass., 1978. 2

[47] Marek Rychlik. Bounded variation and invariant measures. Studia Mathematica, 76:69-80, 1983. 10

[48] David Simmons and Mariusz Urbański. Relative equilibrium states and dimensions of fiberwise invariant measures for random distance expanding maps. Stochastics and Dynamics, 14:1350015, May 2013. 2, 3, 51 
[49] Manuel Stadlbauer. On random topological markov chains with big images and preimages. Stochastics and Dynamics, 10(01):77-95, March 2010. Publisher: World Scientific Publishing Co. 2

[50] Manuel Stadlbauer. Coupling methods for random topological Markov chains. Ergodic Theory and Dynamical Systems, 37(3):971-994, May 2017. 2

[51] Manuel Stadlbauer, Shintaro Suzuki, and Paulo Varandas. Thermodynamic formalism for random nonuniformly expanding maps. arXiv:2006.03749 /math/, June 2020. arXiv: 2006.03749. 2

[52] Manuel Stadlbauer, Paulo Varandas, and Xuan Zhang. Quenched and annealed equilibrium states for random Ruelle expanding maps and applications. April 2020. arXiv: 2004.04763. 2

[53] Mariusz Urbański and Anna Zdunik. Random non-hyperbolic exponential maps. arXiv:1805.08050 [math], May 2018. 3

[54] Paulo Varandas and Marcelo Viana. Existence, uniqueness and stability of equilibrium states for nonuniformly expanding maps. Annales de l'IHP Analyse non linéaire, 27(2):555-593, 2010. 2

School of Mathematics and Statistics, University of New South Wales, Sydney, NSW 2052, Australia

Email address: j.atnip@unsw.edu.au

School of Mathematics and Statistics, University of New South Wales, Sydney, NSW 2052, Australia

Email address: g.froyland@unsw.edu.au

School of Mathematics and Physics, The University of Queensland, St Lucia, QLD 4072, Australia

Email address: cecilia.gt@uq.edu.au

Aix Marseille Université, Université de Toulon, CNRS, CPT, 13009 Marseille, France

Email address: vaienti@cpt.univ-mrs.fr 